\title{
A multilayer shallow water system for polydisperse sedimentation
}

\author{
E.D. Fernández-Nieto $\stackrel{*}{;}$ E.H. Koné $†$ T. Morales de Luna $\ddagger$ R. Bürger ${ }^{\S}$
}

December 10, 2012

\begin{abstract}
This work considers the flow of a fluid containing one disperse substance consisting of small particles that belong to different species differing in size and density. The flow is modelled by combining a multilayer shallow water approach with a polydisperse sedimentation process. This technique allows one to keep information on the vertical distribution of the solid particles in the mixture, and thereby to model the segregation of the particle species from each other, and from the fluid, taking place in the vertical direction of the gravity body force only. This polydisperse sedimentation process is described by the well-known Masliyah-Lockett-Bassoon (MLB) velocity functions. The resulting multilayer sedimentation-flow model can be written as a hyperbolic system with nonconservative products. The definitions of the nonconservative products are related to the hydrostatic pressure and to the mass and momentum hydrodynamic transfer terms between the layers. For the numerical discretization a strategy of two steps is proposed, where the first one is also divided into two parts. In the first step, instead of approximating the complete model, we approximate a reduced model with a smaller number of unknowns. Then, taking advantage of the fact that the concentrations are passive scalars in the system, we approximate the concentrations of the different species by an upwind scheme related to the numerical flux of the total concentration. In the second step, the effect of the transference terms defined in terms of the MLB model is introduced. These transfer terms are approximated by using a numerical flux function used to discretize the $1 \mathrm{D}$ vertical polydisperse model (see Bürger, García, Karlsen and Towers, J. Eng. Math. 60 (2008), 387-425). Finally, some numerical examples are presented. Numerical results suggest that the multilayer shallow water model could be adequate in situations where the settling takes place from a suspension that undergoes horizontal movement.
\end{abstract}

KEY WORDS: sediment; polydisperse; multilayer; shallow water; finite volume

\section{Introduction}

\subsection{Scope}

Numerous applications involve the flow of a mixture of one substance, for example solid mineral particles or oil droplets in an emulsion, dispersed in a continuous phase, say a liquid or gas.

\footnotetext{
* Corresponding author. Dpto. Matemática Aplicada I. ETS Arquitectura - Universidad de Sevilla. Avda. Reina Mercedes N. 2. 41012-Sevilla, Spain. (edofer@us.es)

${ }_{\dagger}^{\dagger}$ Dpto. Matemática Aplicada I. ETS Arquitectura - Universidad de Sevilla. Avda. Reina Mercedes N. 2. 41012-Sevilla, Spain. (ekone@us.es)

${ }^{\ddagger}$ Dpto. de Matemáticas. Universidad de Córdoba. Campus de Rabanales. 14071 Córdoba. (tomas.morales@uco.es)

${ }^{\S} \mathrm{CI}^{2} \mathrm{MA} \&$ Departamento de Ingeniería Matemática, Facultad de Ciencias Físicas y Matemáticas, Universidad de Concepción, Casilla 160-C, Correo 3, Concepción, Chile. (rburger@ing-mat.udec.cl)
} 
In many cases, the disperse substance consists of small particles that belong to different species differing in some characteristic quantity such as size or density. As a consequence, the polydisperse mixture does not move as one phase; rather, the different species segregate and create areas of different composition. In many applications, practitioners are most interested in this differential movement of the species, which is frequently described by spatially one-dimensional models. In most circumstances, the diameter of the particles is small, which justifies identifying each species with a continuous phase. The resulting models usually give rise to a strongly coupled system of nonlinear first-order conservation laws for the volume fractions of the solids species.

In many other applications, we are not only interested in this differential movement of the species but also in the fluid dynamics of the flow convecting the particulate suspensions, for instance the transport of soils, silt and sand in rivers and estuaries. In those cases, a full three-dimensional (3D) model could be considered, but the computational cost of solving such models is largely increased since in two or three space dimensions, not only a multi-dimensional version of the above-mentioned system of conservation laws, but also additional equations of motion (e.g., the Stokes or Navier-Stokes system) for the flow field of the mixture must be solved.

A common approach to model suspended sediment transport in shallow regimes is to use a SaintVenant or shallow water model combined with passive transport equations for the different species. These models are obtained by averaging the original 3D equations along the height of the fluid and allow one to simulate sediment transport with a relative small computational cost (see, e,g, $[16,21,22,28])$. The drawback of these models is that they only take into account the mean depth-average concentration of solid particles in suspension. Thus, the vertical distribution and settling of the particles suspended within the fluid is not described.

The objective of this paper is to derive and implement a computational model for polydisperse sedimentation that takes into account the differential movement of the species as well as the dynamics of the flow. This will be achieved by a multilayer Saint-Venant approach (see for example $[1,3,4,6,33])$.

As is shown in $[2,4]$, numerical simulations by using a multilayer approach allow one to recover interesting properties that are not observed when using just a hydrostatic shallow water model. Moreover, numerical results obtained with the multilayer approach show a good agreement with those obtained for a 3D free-surface Navier-Stokes system when the hydrostatic assumption is in effect. In $[2,4]$ it is shown that the multilayer approach provides an alternative to the solution of the free-surface Navier-Stokes system, leading to a precise description of the vertical profile of the horizontal velocity while preserving the robustness and the computational efficiency of the usual Saint-Venant system. The multilayer technique has also been used for for density-stratified flows in [5] where similar conclusions have been obtained. We employ this technique for the simulation of free-surface fluids with polydisperse sedimentation. This technique allows us to keep information on the vertical distribution of the mixture.

On the other hand, it is difficult to compare exactly the computational cost of different numerical techniques, for example it depends on the efficiency of the coding process. But an interesting property of the multilayer approach is that it enables one to approximate free surface flows without an extra difficulty. Then, the multilayer model can be computationally less expensive than some other numerical techniques to approach the full 3D model for free-surface flows. For instance, in [4] the authors remark that a comparable test using 6 layers (1452 nodes, 2620 triangles for the 2D mesh) takes a CPU time of 10 minutes for the multilayer and 33 minutes for the hydrostatic Navier-Stokes solver.

\subsection{Related work}

Mathematical models for the one-dimensional sedimentation of polydisperse suspensions are important to many applications in chemical engineering, mineral processing, wastewater treatment, medicine, geology, and other areas; see, for example, [17, 23, 32, 34, 36, 37] for applications and $[8,10]$ for mathematical treatments. On the other hand, experimental and theoretical analyses of 
two- or three-dimensional scenarios, where the convective sediment transport is important, include $[9,14,18,24,25]$.

Multilayer Saint-Venant models have been used to study flows with large friction coefficients, with significant water depth and/or with important wind effects, among others (see for example [3], [6], [33]). In these cases, the standard shallow water system is considered invalid since the horizontal velocity can hardly be approximated by a vertically constant velocity in the whole domain.

The multilayer approach consists in subdividing, in the vertical direction, the domain into layers. This way, the multilayer Saint-Venant system derived in [1] consists in a set of coupled Saint-Venant systems for each layer. It is noteworthy that the layers are assumed here to be advected by the flow. Then, it is considered that no mass exchange occurs between neighboring layers making the model physically close to non-miscible fluids simulation. It is also extended to 3D computations of free surface flows with friction and viscosity effects in [4].

A different multilayer model using a formal asymptotic analysis of the two-dimensional (2D) incompressible Navier-Stokes equations with a hydrostatic framework is proposed by Audusse et al. in [6]. Each layer is described by its height and by a vertically constant horizontal velocity. The main improvement is that mass and momentum exchange between the layers are allowed. In order to close the system, the height of the layer is related to the total height of the fluid. Then, the unknowns of the system are the total height of the fluid and a constant horizontal velocity at each layer. The vertical velocity can be computed by postprocessing, taking into account the incompressibility of the fluid.

In [5] Audusse et al. present the extension of this model for free surface density-stratified flows. It approximates formally the Navier-Stokes equations with variable density, when it varies depending on a quantity such as temperature or salinity.

Taking into account the non-hydrostatic pressure associated with a vertical acceleration, a nonhydrostatic multilayer Saint-Venant system is obtained in [33] by an asymptotic analysis of the 2D incompressible Euler equations. In addition to the total fluid height and the horizontal velocities, the vertical velocities and pressures in the layers are the unknowns of the system. These last ones are also vertically averaged as the horizontal velocities.

\subsection{Outline of the paper}

The remainder of the paper is organized as follows. In Section 2 the polydisperse sedimentation model of [8] is outlined. In Section 3 we deduce the multilayer model to study polydisperse sedimentation. A numerical approach of the proposed model is considered in Section 4. In Section 5 we present several numerical tests. Finally, the conclusions of the paper are set in Section 6 . In Appendix A and Appendix B we develop some of the computations necessary to rewrite the model under the structure of an hyperbolic system with conservative components, source terms and nonconservative products.

\section{A polydisperse sedimentation model}

Let us consider $N \in \mathbb{N}^{*}$ species of spherical solid particles dispersed in a viscous fluid. For each solid species $j, j=1, \ldots, N$, we denote by $\phi_{j}, \rho_{j}, d_{j}$, and $\boldsymbol{v}_{j}=\left(u_{j}, \varpi_{j}\right), j=1, \ldots, N$, its volumetric concentration, density, particle diameter, and phase velocity, respectively. Here $u_{j}$ and $\varpi_{j}$ stand for the horizontal and vertical components of the phase velocities, respectively. The same notation is used for the fluid indexed by $j=0$. The model is outlined in detail in [8], and will be used here in final form only. Moreover, we assume that effects of sediment compressibility can be neglected. The model is based on the continuity and linear momentum balance equations for the $N$ solid species and the fluid. The continuity equations are given by

$$
\partial_{t} \phi_{j}+\nabla \cdot\left(\phi_{j} \boldsymbol{v}_{j}\right)=0, \quad j=0, \ldots, N .
$$


Taking into account that $\phi=1-\phi_{0}$, where $\phi:=\phi_{1}+\cdots+\phi_{N}$ denotes the total solids concentration, we see by summing all equations in (2.1) that the volume average velocity of the mixture

$$
\boldsymbol{q}:=(u, \varpi):=\phi_{0} \boldsymbol{v}_{0}+\phi_{1} \boldsymbol{v}_{1}+\cdots+\phi_{N} \boldsymbol{v}_{N}=(1-\phi) \boldsymbol{v}_{0}+\phi_{1} \boldsymbol{v}_{1}+\cdots+\phi_{N} \boldsymbol{v}_{N}
$$

satisfies the simple mass balance of the mixture

$$
\nabla \cdot \boldsymbol{q}=0
$$

On the other hand, from the equations of conservation of linear momentum one obtains by introducing suitable constitutive assumptions, followed by a dimensional analysis (see [8]), the expression

$$
\boldsymbol{v}_{j}=\boldsymbol{q}+v_{j}^{\mathrm{MLB}}(\Phi) \boldsymbol{e}_{z}, \quad j=1, \ldots, N,
$$

where $\Phi:=\left(\phi_{1}, \ldots, \phi_{N}\right)^{\mathrm{T}}$ and $\boldsymbol{e}_{z}$ is the upward-pointing unit vector. The hindered settling velocities $v_{j}^{\mathrm{MLB}}(\Phi)$ are those given in one space dimension by Masliyah [27] and Lockett and Bassoon [26] ("MLB model"), namely

$$
v_{j}^{\mathrm{MLB}}(\Phi)=\mu V(\phi)\left[\delta_{j}\left(\bar{\rho}_{j}-\overline{\boldsymbol{\rho}}^{\mathrm{T}} \Phi\right)-\sum_{l=1}^{N} \delta_{l} \phi_{l}\left(\bar{\rho}_{l}-\overline{\boldsymbol{\rho}}^{\mathrm{T}} \Phi\right)\right], \quad j=1, \ldots, N,
$$

where we define $\bar{\rho}_{j}:=\rho_{j}-\rho_{0}$ for $j=1, \ldots, N, \bar{\rho}:=\left(\bar{\rho}_{1}, \ldots, \bar{\rho}_{N}\right)^{\mathrm{T}}, \mu=-g d_{1}^{2} /\left(18 \mu_{\mathrm{f}}\right)$, where $g$ is the acceleration of gravity and $\mu_{\mathrm{f}}$ is the viscosity of pure fluid, and $\delta_{j}:=d_{j}^{2} / d_{1}^{2}$. Moreover, $V=V(\phi)$ is a so-called hindered settling factor, which may be chosen as follows [31]:

$$
V(\phi)= \begin{cases}(1-\phi)^{\lambda-2} & \text { if } \Phi \in \overline{\mathcal{D}}_{\phi_{\max }}, \quad \lambda>2, \\ 0 & \text { otherwise }\end{cases}
$$

where $\overline{\mathcal{D}}_{\phi_{\max }}$ is the closure of the set

$$
\mathcal{D}_{\phi_{\max }}:=\left\{\Phi \in \mathbb{R}^{N} \mid \phi_{1}>0, \ldots, \phi_{N}>0, \phi:=\phi_{1}+\cdots+\phi_{N}<\phi_{\max }\right\},
$$

where $\phi_{\max }$ is a maximal solids concentration. Note that in one (vertical) space dimension, (2.2) means that $q$ is spatially constant (with $q=0$ for batch settling in a column), and the equations (2.1) for $j=1, \ldots, N$ with the fluxes (2.3), (2.4) form a closed system, with no other flow variables involved; for this reason the MLB model is also called kinematic in that case.

For future reference we remark that the vertical velocities of particles are expressed as a combination of the vertical volume-average velocity of the mixture $\varpi:=\phi_{1} \varpi_{1}+\cdots+\phi_{N} \varpi_{N}$ and the fluxes $f_{j}(\Phi)=\phi_{j} v_{j}^{\mathrm{MLB}}(\Phi)$, where $v_{j}^{\mathrm{MLB}}(\Phi)$ is given by the MLB model (2.4):

$$
\phi_{j} \varpi_{j}=\phi_{j} \varpi+f_{j}(\Phi), \quad j=1, \ldots, N .
$$

Moreover we get the following equality:

$$
\sum_{j=1}^{N} f_{j}(\Phi)=(1-\phi)\left(\varpi-\varpi_{0}\right)=\phi_{0}\left(\varpi-\varpi_{0}\right) .
$$

From (2.7) we may define a similar relation for the fluid volume fraction:

$$
\phi_{0} \varpi_{0}=\phi_{0} \varpi+f_{0}(\Phi) \quad \text { with } \quad f_{0}(\Phi)=-\sum_{j=1}^{N} f_{j}(\Phi) .
$$


We conclude by recalling that under the present assumptions, the mass equation for the solids species can be written as

$$
\partial_{t} \phi_{j}+\partial_{x}\left(\phi_{j} u_{j}\right)+\partial_{z}\left(\phi_{j} \varpi+f_{j}(\Phi)\right)=0, \quad j=1, \ldots, N .
$$

The model also involves the linear momentum balance equations for the solid phases [8]. Assuming that the advective acceleration terms are significant while sediment compressibility and viscous stresses are neglected, we obtain the following form of these balance equations:

$$
\rho_{j}\left(\partial_{t}\left(\phi_{j} \boldsymbol{v}_{j}\right)+\nabla \cdot\left(\phi_{j} \boldsymbol{v}_{j} \otimes \boldsymbol{v}_{j}\right)\right)=-\rho_{j} \phi_{j} g \boldsymbol{k}-\phi_{j} \nabla p, \quad j=1, \ldots, N .
$$

We comment that the assumptions stated in [8], which lead to (2.3) but are not made explicit herein, include that the solid-fluid relative velocity between particle species $j$ and the fluid, defined by $\boldsymbol{r}_{j}:=\boldsymbol{v}_{j}-\boldsymbol{v}_{0}, \quad j=1, \ldots, N$, has a nonzero component in the vertical direction aligned with $\boldsymbol{e}_{z}$ only, i.e., at any point all solid phases and the fluid move at the same horizontal velocity. In the following lemma we show that this property can be recovered from (2.3).

Lemma 1. Assume that the velocities of the solid species are given by (2.3). Then the horizontal velocity of each species coincides with that of the fluid, that is, we may define $u_{j}:=u$ for all $j=0,1, \ldots, N$.

Proof.

We show that $\boldsymbol{r}_{j}=\left(u_{j}-u_{0}, \varpi_{j}-\varpi_{0}\right)^{\mathrm{T}}$ is a multiple of $\boldsymbol{e}_{z}=(0,1)^{\mathrm{T}}$ for $j=1, \ldots, N$ and $\phi<1$. To this end, note that (2.3) implies that

$$
\boldsymbol{r}_{j}-\sum_{i=1}^{N} \phi_{i} \boldsymbol{r}_{i}=v_{j}^{\mathrm{MLB}}(\Phi) \boldsymbol{e}_{z}, \quad j=1, \ldots, N .
$$

As in [13] we may use a Sherman-Morrison formula to conclude that the unique solution of (2.10) is given by

$$
\boldsymbol{r}_{j}=\left(v_{j}^{\mathrm{MLB}}(\Phi)-\frac{\phi_{j}}{1-\phi} \sum_{i=1}^{n} \phi_{i} v_{i}^{\mathrm{MLB}}(\Phi)\right) \boldsymbol{e}_{z}, \quad j=1, \ldots, N
$$

\section{A multilayer approach}

In order to introduce a multilayer system, the mixture domain is divided along the vertical direction into $M \in \mathbb{N}^{*}$ layers of thickness $h_{\alpha}(t, x)$ with $M+1$ interfaces defined by $z_{\alpha+1 / 2}(t, x)$ for $\alpha=$ $0,1, \ldots, M$ (see Figure 1). Denoting by $z_{\mathrm{B}}:=z_{1 / 2}$ and $z_{\mathrm{S}}:=z_{M+1 / 2}$ the bottom and the free surface interfaces, respectively, we have $h_{\alpha}=z_{\alpha+1 / 2}-z_{\alpha-1 / 2}$ and $z_{\alpha+1 / 2}=z_{\mathrm{B}}+h_{1}+\cdots+h_{\alpha}$ for $\alpha=1, \ldots, M$, and then the height of the fluid is given by $h=z_{\mathrm{S}}-z_{\mathrm{B}}=h_{1}+\cdots+h_{M}$.

Notation 1. For $\alpha=1, \ldots, M$ we denote by $\left.I_{\alpha}=I_{\alpha}(t, x):=\right] z_{\alpha-1 / 2}(t, x), z_{\alpha+1 / 2}(t, x)[$ the varying one-dimensional vertical interval and for a given function $a(t, x, z)$, we denote the vertically averaged and the vertical fluctuation functions, respectively, by

$$
a_{\alpha}(t, x):=\frac{1}{h_{\alpha}} \int_{I_{\alpha}} a(t, x, z) \mathrm{d} z \quad \text { and } \quad \widehat{a_{\alpha}}(t, x, z):=a(t, x, z)-a_{\alpha}(t, x) .
$$

In addition, $a_{\alpha+1 / 2}$ denotes the restriction of the function a to the interface $z=z_{\alpha+1 / 2}$.

Remark 1. From (3.1) we obtain the following identities:

$$
\int_{I_{\alpha}} \widehat{a_{\alpha}}(t, x, z) \mathrm{d} z=0
$$




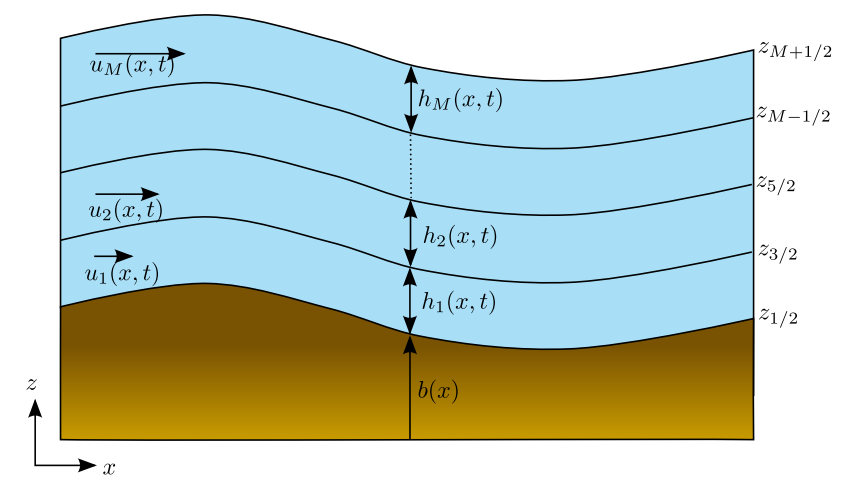

Figure 1: Sketch of the multilayer division of the mixture domain.

$$
\begin{aligned}
& \int_{I_{\alpha}} a b \mathrm{~d} z=h_{\alpha} a_{\alpha} b_{\alpha}+\int_{I_{\alpha}} \widehat{a_{\alpha}} \widehat{b_{\alpha}} \mathrm{d} z, \\
& \int_{I_{\alpha}} a b c \mathrm{~d} z=h_{\alpha} a_{\alpha} b_{\alpha} c_{\alpha}+\int_{I_{\alpha}}\left(c_{\alpha} \widehat{a_{\alpha}} \widehat{b_{\alpha}}+\widehat{c_{\alpha}} a b\right) \mathrm{d} z .
\end{aligned}
$$

Integrating the mass balance equations (2.1) over $I_{\alpha}$ for all $\alpha=1, \ldots, M$ and $j=0,1, \ldots, N$ and using the Leibniz integration rule, we get

$$
\begin{aligned}
0= & \int_{I_{\alpha}}\left(\partial_{t} \phi_{j}+\partial_{x}\left(\phi_{j} u\right)+\partial_{z}\left(\phi_{j} \varpi_{j}\right)\right) \mathrm{d} z \\
= & \partial_{t} \int_{I_{\alpha}} \phi_{j} \mathrm{~d} z+\partial_{x} \int_{I_{\alpha}} \phi_{j} u \mathrm{~d} z \\
& -\Delta_{-}^{\alpha}\left(\phi_{j, \alpha+1 / 2} \partial_{t} z_{\alpha+1 / 2}\right)-\Delta_{-}^{\alpha}\left(\left(\phi_{j} u\right)_{\alpha+1 / 2} \partial_{x} z_{\alpha+1 / 2}\right)+\left[\phi_{j} \varpi_{j}\right]_{z_{\alpha-1 / 2}}^{z_{\alpha+1 / 2}},
\end{aligned}
$$

where we define the difference operators $\Delta_{+}^{\alpha}$ and $\Delta_{-}^{\alpha}$ via $\Delta_{+}^{\alpha} p_{j, \alpha}:=p_{j, \alpha+1}-p_{j, \alpha}$ and $\Delta_{-}^{\alpha} p_{j, \alpha}:=$ $p_{j, \alpha}-p_{j, \alpha-1}$, respectively. Then (2.6) and Remark 1 lead to the equality

$$
\partial_{t}\left(h_{\alpha} \phi_{j, \alpha}\right)+\partial_{x}\left(h_{\alpha} \phi_{j, \alpha} u_{\alpha}\right)+\partial_{x} \int_{I_{\alpha}} \widehat{\phi_{j, \alpha}} \widehat{u_{\alpha}} \mathrm{d} z=\Delta_{-}^{\alpha}\left(\phi_{j, \alpha+1 / 2} G_{\alpha+1 / 2}-f_{j, \alpha+1 / 2}(\Phi)\right)
$$

for $j=0,1, \ldots, N$ and $\alpha=1, \ldots, M$, where we define for $\alpha=0,1, \ldots, M$

$$
\begin{aligned}
G_{\alpha+1 / 2} & :=\partial_{t} z_{\alpha+1 / 2}+u_{\alpha+1 / 2} \partial_{x} z_{\alpha+1 / 2}-\varpi_{\alpha+1 / 2}, \\
f_{0, \alpha+1 / 2}(\Phi) & :=-\phi_{0, \alpha+1 / 2}\left(\varpi_{\alpha+1 / 2}-\varpi_{0, \alpha+1 / 2}\right) .
\end{aligned}
$$

Assumption 1. We assume that the thickness of each layer is small enough to neglect the vertical fluctuations of the horizontal velocities and concentrations inside. That is, we define

$$
\varepsilon=\max _{(t, x)}\left|I_{\alpha}(t, x)\right|, \quad \text { for all } \alpha
$$

and we suppose that $\varepsilon$ is a very small value. Then we have

$$
\partial_{x} \int_{I_{\alpha}} \widehat{\phi_{j, \alpha}} \widehat{u_{\alpha}} \mathrm{d} z=\mathcal{O}(\varepsilon) \text { for all } \alpha \text { and all } j
$$

We shall neglect the terms $\mathcal{O}(\varepsilon)$ in the further development. 
In light of Assumption 1, we obtain from (3.2) the following equations:

$$
\partial_{t}\left(h_{\alpha} \phi_{j, \alpha}\right)+\partial_{x}\left(h_{\alpha} \phi_{j, \alpha} u_{\alpha}\right)=\Delta_{-}^{\alpha} H_{j, \alpha+1 / 2}(\Phi), \quad \alpha=1, \ldots, M, \quad j=0,1, \ldots, N,
$$

where we define

$$
H_{j, \alpha+1 / 2}(\Phi):=\phi_{j, \alpha+1 / 2} G_{\alpha+1 / 2}-f_{j, \alpha+1 / 2}(\Phi) \quad \text { for } \alpha=0,1, \ldots, M \text { and } j=0,1, \ldots, N .
$$

Next, in view of the equalities

$$
\sum_{j=0}^{N} \phi_{j, \alpha+1 / 2}=1 \quad \text { for all } \alpha=0,1, \ldots, M
$$

we sum the equations (3.3) over $j=0,1, \ldots, N$ and take into account (2.8) to find the following equations concerning the thickness of the layers:

$$
\partial_{t} h_{\alpha}+\partial_{x}\left(h_{\alpha} u_{\alpha}\right)=\Delta_{-}^{\alpha} G_{\alpha+1 / 2}, \quad \alpha=1, \ldots, M
$$

Moreover, summing the horizontal components of equation (2.9) over $j=0,1, \ldots, N$ and integrating over $I_{\alpha}$ for all $\alpha=1, \ldots, M$, we obtain by the Leibniz integration rule the following equation, where $p_{\mathrm{T}}$ denotes the total pressure:

$$
\begin{aligned}
& \partial_{t} \int_{I_{\alpha}} \rho(\Phi) u \mathrm{~d} z+\partial_{x} \int_{I_{\alpha}} \rho(\Phi) u^{2} \mathrm{~d} z+\sum_{j=0}^{N} \rho_{j}\left[\phi_{j} \varpi_{j} u\right]_{z_{\alpha-1 / 2}}^{z_{\alpha+1 / 2}} \\
& =-\partial_{x} \int_{I_{\alpha}} p_{\mathrm{T}} \mathrm{d} z+\Delta_{-}^{\alpha}\left((\rho(\Phi) u)_{\alpha+1 / 2}\left(\partial_{t} z_{\alpha+1 / 2}+u_{\alpha+1 / 2} \partial_{x} z_{\alpha+1 / 2}\right)\right)+\Delta_{-}^{\alpha}\left(p_{\mathrm{T}, \alpha+1 / 2} \partial_{x} z_{\alpha+1 / 2}\right) .
\end{aligned}
$$

Let us denote the mixture density per layer by

$$
\rho_{\alpha}(\Phi):=\sum_{j=0}^{N} \rho_{j} \phi_{j, \alpha}=\rho_{0}+\sum_{j=1}^{N}\left(\rho_{j}-\rho_{0}\right) \phi_{j, \alpha} .
$$

Then we have

$$
\begin{aligned}
& \partial_{t}\left(h_{\alpha} \rho_{\alpha}(\Phi) u_{\alpha}\right)+\partial_{x}\left(h_{\alpha} \rho_{\alpha}(\Phi) u_{\alpha}^{2}\right)+\partial_{x} \int_{I_{\alpha}} p_{\mathrm{T}} \mathrm{d} z+\partial_{t} \int_{I_{\alpha}} \widehat{\rho_{\alpha}(\Phi)} \widehat{u_{\alpha}} \mathrm{d} z \\
& \quad+\partial_{x} \int_{I_{\alpha}}\left(\rho_{\alpha}(\Phi){\widehat{u_{\alpha}}}^{2}+\widehat{\rho_{\alpha}(\Phi)} u^{2}\right) \mathrm{d} z \\
& =\Delta_{-}^{\alpha}\left((\rho(\Phi) u)_{\alpha+1 / 2}\left(\partial_{t} z_{\alpha+1 / 2}+u_{\alpha+1 / 2} \partial_{x} z_{\alpha+1 / 2}\right)\right)-\sum_{j=1}^{N}\left[\rho_{j}\left(\phi_{j} \varpi+f_{j}(\Phi)\right) u\right]_{z_{\alpha-1 / 2}}^{z_{\alpha+1 / 2}} \\
& \quad-\rho_{0}\left[\phi_{0} \varpi_{0} u\right]_{z_{\alpha-1 / 2}}^{z_{\alpha+1 / 2}}+\Delta_{-}^{\alpha}\left(p_{\mathrm{T}, \alpha+1 / 2} \partial_{x} z_{\alpha+1 / 2}\right) \\
& =\sum_{j=0}^{N} \rho_{j} \Delta_{-}^{\alpha}\left(u_{\alpha+1 / 2} H_{j, \alpha+1 / 2}(\Phi)\right)+\Delta_{-}^{\alpha}\left(p_{\mathrm{T}, \alpha+1 / 2} \partial_{x} z_{\alpha+1 / 2}\right) .
\end{aligned}
$$

By Assumption 1, since we neglect the vertical fluctuation functions and since

$$
\widehat{\rho_{\alpha}(\Phi)}=\sum_{j=0}^{N} \rho_{j} \widehat{\phi_{j, \alpha}}
$$


we have

$$
\partial_{t} \int_{I_{\alpha}} \widehat{\rho_{\alpha}(\Phi)} \widehat{u_{\alpha}} \mathrm{d} z+\partial_{x} \int_{I_{\alpha}}\left(\rho_{\alpha}(\Phi){\widehat{u_{\alpha}}}^{2}+\widehat{\rho_{\alpha}(\Phi)} u^{2}\right) \mathrm{d} z=\mathcal{O}(\varepsilon) \quad \text { for all } \alpha=1, \ldots, M .
$$

If we neglect the terms of order $\mathcal{O}(\varepsilon)$, we get the following equations:

$$
\begin{aligned}
& \partial_{t}\left(h_{\alpha} \rho_{\alpha}(\Phi) u_{\alpha}\right)+\partial_{x}\left(h_{\alpha} \rho_{\alpha}(\Phi) u_{\alpha}^{2}\right)+\partial_{x}\left(h_{\alpha} p_{\mathrm{T}, \alpha}\right) \\
& =\sum_{j=0}^{N} \rho_{j} \Delta_{-}^{\alpha}\left(u_{\alpha+1 / 2} H_{j, \alpha+1 / 2}(\Phi)\right)+\Delta_{-}^{\alpha}\left(p_{\mathrm{T}, \alpha+1 / 2} \partial_{x} z_{\alpha+1 / 2}\right), \quad \alpha=1, \ldots, M
\end{aligned}
$$

Assumption 2. We assume that we have a shallow domain. In this case, the total pressure can be assumed to be hydrostatic, i.e., $\partial_{z} p_{\mathrm{T}}=-\rho(\Phi) g$.

Then for $z \in I_{\alpha}$,

$$
\begin{aligned}
p_{\mathrm{T}}(t, x, z) & =p_{\mathrm{S}}(t, x)+\sum_{\beta=\alpha+1}^{M}\left(\int_{I_{\beta}} \rho(\Phi) g \mathrm{~d} z^{\prime}\right)+\int_{z}^{z_{\alpha+1 / 2}} \rho(\Phi) g \mathrm{~d} z^{\prime} \\
& =p_{\mathrm{S}}(t, x)+g \sum_{\beta=\alpha+1}^{M} h_{\beta} \rho_{\beta}(\Phi)+\int_{z}^{z_{\alpha+1 / 2}} \rho(\Phi) g \mathrm{~d} z^{\prime},
\end{aligned}
$$

where $p_{\mathrm{S}}$ stands for the pressure at the free surface. Then the equality

$$
p_{\mathrm{T}, \alpha+1 / 2}=p_{\mathrm{S}}+\sum_{\beta=\alpha+1}^{M} h_{\beta} \rho_{\beta}(\Phi) g
$$

holds and we have

$$
\begin{aligned}
p_{\mathrm{T}, \alpha}(t, x) & =p_{\mathrm{T}, \alpha+1 / 2}+\frac{1}{h_{\alpha}} \int_{I_{\alpha}} \int_{z}^{z_{\alpha+1 / 2}} \rho(\Phi) g \mathrm{~d} z^{\prime} \mathrm{d} z \\
& =p_{\mathrm{T}, \alpha+1 / 2}+\frac{1}{h_{\alpha}} \int_{I_{\alpha}} \rho_{\alpha}(\Phi) g\left(z_{\alpha+1 / 2}-z\right) \mathrm{d} z+\frac{1}{h_{\alpha}} \int_{I_{\alpha}} \int_{z}^{z_{\alpha+1 / 2}} \widehat{\rho_{\alpha}(\Phi)} g \mathrm{~d} z^{\prime} \mathrm{d} z .
\end{aligned}
$$

By Assumption 1 we have that

$$
\frac{1}{h_{\alpha}} \int_{I_{\alpha}} \int_{z}^{z_{\alpha+1 / 2}} \widehat{\rho_{\alpha}(\Phi)} g \mathrm{~d} z^{\prime} \mathrm{d} z=\mathcal{O}(\varepsilon) .
$$

By neglecting this term of $\mathcal{O}(\varepsilon)$ we have

$$
p_{\mathrm{T}, \alpha}(t, x)=p_{\mathrm{S}}+g\left(\frac{1}{2} h_{\alpha} \rho_{\alpha}(\Phi)+\sum_{\beta=\alpha+1}^{M} h_{\beta} \rho_{\beta}(\Phi)\right) .
$$

In addition, we point out the expansion

$$
\Delta_{-}^{\alpha}\left(p_{\mathrm{T}, \alpha+1 / 2} \partial_{x} z_{\alpha+1 / 2}\right)=\left(p_{\mathrm{S}}+g \sum_{\beta=\alpha+1}^{M} h_{\beta} \rho_{\beta}(\Phi)\right) \partial_{x} h_{\alpha}-h_{\alpha} \rho_{\alpha} g\left(\partial_{x} z_{\mathrm{B}}+\sum_{\beta=1}^{\alpha-1} \partial_{x} h_{\beta}\right) .
$$


The final model is constituted by the equations (3.3), (3.5), (3.6) and is summarized here:

$$
\left\{\begin{array}{l}
\partial_{t} h_{\alpha}+\partial_{x}\left(h_{\alpha} u_{\alpha}\right)=\Delta_{-}^{\alpha} G_{\alpha+1 / 2} \\
\partial_{t}\left(h_{\alpha} \phi_{j, \alpha}\right)+\partial_{x}\left(h_{\alpha} \phi_{j, \alpha} u_{\alpha}\right)=\Delta_{-}^{\alpha} H_{j, \alpha+1 / 2}(\Phi) \\
\partial_{t}\left(h_{\alpha} \rho_{\alpha}(\Phi) u_{\alpha}\right)+\partial_{x}\left(h_{\alpha} \rho_{\alpha}(\Phi) u_{\alpha}^{2}\right)+\partial_{x}\left(h_{\alpha}\left[p_{\mathrm{S}}+\frac{1}{2} g h_{\alpha} \rho_{\alpha}(\Phi)+g \sum_{\beta=\alpha+1}^{M} h_{\beta} \rho_{\beta}(\Phi)\right]\right) \\
=\sum_{j=0}^{N} \rho_{j} \Delta_{-}^{\alpha}\left(u_{\alpha+1 / 2} H_{j, \alpha+1 / 2}(\Phi)\right)+\left(p_{\mathrm{S}}+g \sum_{\beta=\alpha+1}^{M} h_{\beta} \rho_{\beta}(\Phi)\right) \partial_{x} h_{\alpha} \\
\quad-h_{\alpha} \rho_{\alpha} g\left(\partial_{x} z_{\mathrm{B}}+\sum_{\beta=1}^{\alpha-1} \partial_{x} h_{\beta}\right)
\end{array}\right.
$$

where $H_{j, \alpha+1 / 2}(\Phi)$ is defined by (3.4) and $f_{j, \alpha+1 / 2}(\Phi)$ is given by (2.4). The quantities $u_{\alpha+1 / 2}$ and $G_{\alpha+1 / 2}$ are specified in what follows. Let us remark that the positions of the artificial interfaces $z_{\alpha+1 / 2}$ must be set. In this paper, they are defined by considering layers having thickness proportional to the total height. As we see in the rest of the section, this closure implies the definition of the transfer terms $G_{\alpha+1 / 2}$ for all $\alpha$.

To that end, we set the following hypothesis on the heights of the layers.

Assumption 3. For $\alpha=1, \ldots, M, h_{\alpha}=l_{\alpha} h$ with $l_{\alpha}$ a positive constant. Hence we have

$$
\sum_{\alpha=1}^{M} l_{\alpha}=1 .
$$

From that hypothesis, we get the global continuity equation

$$
\partial_{t} h+\partial_{x}\left(h \sum_{\beta=1}^{M} l_{\beta} u_{\beta}\right)=G_{M+1 / 2}-G_{1 / 2} .
$$

Now, the system (3.7) reduces to

$$
\left\{\begin{array}{l}
\partial_{t} h+\partial_{x}\left(h \sum_{\beta=1}^{M} l_{\beta} u_{\beta}\right)=G_{M+1 / 2}-G_{1 / 2}, \\
\partial_{t}\left(h \phi_{j, \alpha}\right)+\partial_{x}\left(h \phi_{j, \alpha} u_{\alpha}\right)=\frac{1}{l_{\alpha}} \Delta_{-}^{\alpha}\left(H_{j, \alpha+1 / 2}(\Phi)\right), \\
\partial_{t}\left(h \rho_{\alpha}(\Phi) u_{\alpha}\right)+\partial_{x}\left(h \rho_{\alpha}(\Phi) u_{\alpha}^{2}\right)+\partial_{x}\left(h p_{\mathrm{S}}+g h^{2}\left[\frac{1}{2} l_{\alpha} \rho_{\alpha}(\Phi)+\sum_{\beta=\alpha+1}^{M} l_{\beta} \rho_{\beta}(\Phi)\right]\right) \\
=\sum_{j=0}^{N} \frac{\rho_{j}}{l_{\alpha}} \Delta_{-}^{\alpha}\left(u_{\alpha+1 / 2} H_{j, \alpha+1 / 2}(\Phi)\right)+\left(p_{\mathrm{S}}+g h \sum_{\beta=\alpha+1}^{M} l_{\beta} \rho_{\beta}(\Phi)\right) \partial_{x} h \\
\quad-g h \rho_{\alpha}\left(\partial_{x} z_{\mathrm{B}}+\sum_{\beta=1}^{\alpha-1} l_{\beta} \partial_{x} h\right) .
\end{array}\right.
$$

Moreover, by summing up from 1 to $\alpha$ the first equation of (3.7), taking into account (3.9) and that we have set $h_{\beta}=l_{\beta}$ for all $\beta$, we obtain that

$$
G_{\alpha+1 / 2}=G_{1 / 2}+\sum_{\beta=1}^{\alpha} l_{\beta}\left(\partial_{t} h+\partial_{x}\left(h u_{\beta}\right)\right)
$$




$$
=G_{1 / 2}\left(1-\sum_{\beta=1}^{\alpha} l_{\beta}\right)+G_{M+1 / 2}\left(\sum_{\beta=1}^{\alpha} l_{\beta}\right)+\sum_{\beta=1}^{\alpha} l_{\beta}\left(\partial_{x}\left(h u_{\beta}\right)-\sum_{\gamma=1}^{M} \partial_{x}\left(l_{\gamma} h u_{\gamma}\right)\right)
$$

Taking into account that the terms $G_{\alpha+1 / 2}$ represent the transfer between the layers $\alpha$ and $\alpha+1$ across the common interface, a natural assumption is to suppose that there is no transfer between the fluid and the bottom or at the surface. Thus, we assume $G_{1 / 2}=G_{M+1 / 2}=0$. Of course, one could eventually consider variants of the model presented here by changing these definitions. For instance, we could include an interchange of sediment at the bottom interface by effects of erosion and deposition. If we set $G_{1 / 2}=G_{M+1 / 2}=0$, we obtain

$$
G_{\alpha+1 / 2}=\sum_{\gamma=1}^{M} \xi_{\alpha, \gamma} \partial_{x}\left(h u_{\gamma}\right), \quad \alpha=1, \ldots, M,
$$

where for $\alpha, \gamma \in\{1, \ldots, M\}$, we define

$$
\xi_{\alpha, \gamma}:=\sum_{\beta=1}^{\alpha}\left(\delta_{\beta \gamma}-l_{\beta}\right) l_{\gamma}= \begin{cases}\left(1-\left(l_{1}+\cdots+l_{\alpha}\right)\right) l_{\gamma} & \text { if } \gamma \leq \alpha \\ -\left(l_{1}+\cdots+l_{\alpha}\right) l_{\gamma} & \text { otherwise }\end{cases}
$$

where $\delta_{\beta \gamma}$ is the standard Kronecker symbol.

Then, note that system (3.10) has $(N+1) M+1$ unknowns and equations, if $u_{\alpha+1 / 2}$ and $\phi_{j, \alpha+1 / 2}$ are set. To define $u_{\alpha+1 / 2}$ and $\phi_{j, \alpha+1 / 2}$ we may use an approach similar to the one used in [6] by considering an upwind scheme, that is, for $\alpha=0,1, \ldots, M$ and $j=1, \ldots, N$ we set

$$
u_{\alpha+1 / 2}=\left\{\begin{array}{ll}
u_{\alpha+1} & \text { if } G_{\alpha+1 / 2} \geq 0, \\
u_{\alpha} & \text { otherwise }
\end{array} \quad \phi_{j, \alpha+1 / 2}= \begin{cases}\phi_{j, \alpha+1} & \text { if } H_{j, \alpha+1 / 2}(\Phi) \geq 0, \\
\phi_{j, \alpha} & \text { otherwise }\end{cases}\right.
$$

where the superscript $n$ stands for the previous time point $t=t_{n}$. For $\alpha=0,1, \ldots, M$ and $j=1, \ldots, N$ we may rewrite (3.12) as follows:

$$
u_{\alpha+1 / 2}=\varepsilon_{\alpha+1 / 2} u_{\alpha}+\left(1-\varepsilon_{\alpha+1 / 2}\right) u_{\alpha+1}, \quad \phi_{j, \alpha+1 / 2}=\theta_{j, \alpha+1 / 2} \phi_{j, \alpha}+\left(1-\theta_{j, \alpha+1 / 2}\right) \phi_{j, \alpha+1},
$$

where $\varepsilon_{\alpha+1 / 2} \in[0,1]$ and $\theta_{j, \alpha+1 / 2} \in[0,1]$ are given by

$$
\varepsilon_{\alpha+1 / 2}=\frac{1}{2}\left(1-\operatorname{sgn}\left(G_{\alpha+1 / 2}^{n}\right)\right), \quad \theta_{j, \alpha+1 / 2}=\frac{1}{2}\left(1-\operatorname{sgn}\left(H_{j, \alpha+1 / 2}^{n}(\Phi)\right)\right) .
$$

Another possible definition for $u_{\alpha+1 / 2}$ and $\phi_{j, \alpha+1 / 2}$ is to replace the equalities (3.13) by $\varepsilon_{\alpha+1 / 2}=$ $\theta_{j, \alpha+1 / 2}=l_{\alpha} /\left(l_{\alpha}+l_{\alpha+1}\right)$, or even $\varepsilon_{\alpha+1 / 2}=\theta_{j, \alpha+1 / 2}=1 / 2$.

Remark 2. In light of (3.8) we have $\xi_{M, \gamma}=0$ for all $\gamma=1, \ldots, M$. In addition, setting $\xi_{0, \gamma}=0$ for all $\gamma=1, \ldots, M$, we notice that $\xi_{\alpha, \gamma}=\xi_{\alpha-1, \gamma}+\left(\delta_{\alpha \gamma}-l_{\alpha}\right) l_{\gamma}$ for all $\alpha, \gamma=1, \ldots, M$.

By a straightforward computation (see Appendix A) we can rewrite the system (3.10) in the more compact form

$$
\partial_{t} \boldsymbol{w}+\partial_{x} \boldsymbol{F}(\boldsymbol{w})+\boldsymbol{B}(\boldsymbol{w}) \partial_{x} \boldsymbol{w}=\boldsymbol{S}(\boldsymbol{w}) \partial_{x} H+\boldsymbol{G}(\boldsymbol{w}),
$$

where $\boldsymbol{w} \in \mathbb{R}^{n}\left(n \in \mathbb{N}^{*}\right)$ is the unknown vector, $\boldsymbol{F}: \mathbb{R}^{n} \rightarrow \mathbb{R}^{n}$ is a regular vectorial function, $\boldsymbol{B}: \mathbb{R}^{n} \rightarrow \mathcal{M}_{n}(\mathbb{R})$ is a matrix function, where $\mathcal{M}_{n}(\mathbb{R})$ is the space of real $n \times n$ matrices, $\boldsymbol{S}, \boldsymbol{G}$ : $\mathbb{R}^{n} \rightarrow \mathbb{R}^{n}$ are vectorial functions, and $H: \mathbb{R}^{n} \rightarrow \mathbb{R}$ is a real scalar function. The form (3.14) constitutes a classical simplified model type for multiphase or multilayer flows in the literature. More precisely, we introduce the notation $r_{j, \alpha}:=\phi_{j, \alpha} h$ for $j=0,1, \ldots, N$ and $q_{\alpha}:=\rho_{\alpha}(\Phi) h u_{\alpha}$, and define the vector

$$
\boldsymbol{w}:=(h, \boldsymbol{q}, \boldsymbol{r})^{\mathrm{T}} \in \mathbb{R}^{((N+1) M+1)}
$$


where

$$
\begin{gathered}
h \in \mathbb{R}, \quad \boldsymbol{q}=\left(q_{1}, q_{2}, \ldots, q_{M}\right)^{\mathrm{T}} \in \mathbb{R}^{M}, \\
\boldsymbol{r}=\left(r_{1,1}, r_{2,1}, \ldots, r_{N, 1}, \ldots, r_{1, \alpha}, r_{2, \alpha}, \ldots, r_{N, \alpha}, \ldots, r_{1, M}, r_{2, M}, \ldots, r_{N, M}\right)^{\mathrm{T}} \in \mathbb{R}^{N M} .
\end{gathered}
$$

The algebraic expressions of the different terms in (3.14) for the current multilayer model are detailed in Appendix A.

Remark 3. The multilayer system approximates the full 3D hydrostatic free-surface polydisperse system, which in general is not a hyperbolic system. Some results about hyperbolicity can be found for the $1 D$ vertical polydisperse system in [10, 20]. For example, it was shown that the MLB model is strictly hyperbolic for arbitrary $N$ species with equal-density particles. On the other hand, for multilayer models, to our knowledge, hyperbolicity is an open problem. In [6] the authors prove hyperbolicity when applied to approximate the hydrostatic Navier-Stokes equations with constant density for the case of two layers.

\section{Numerical approach}

The approach is based on a standard finite volume method combined with a two-step splitting procedure. The splitting consists in ruling out, from the first step, the contribution of the vertical numerical fluxes $f_{j, \alpha+1 / 2}(\Phi)$ for $j=1, \ldots, N$ and $\alpha=0,1, \ldots, M$. However, since the number of variables for the models is $(N+1) M+1$, the size of the system is considerable, especially for large numbers of layers and particle species. Therefore, to reduce the computational cost, instead of solving (3.14) directly, we introduce the variables

$$
m_{\alpha}=\rho_{\alpha}(\Phi) h .
$$

From (3.14) we obtain the following system:

$$
\left\{\begin{array}{l}
\partial_{t} h+\partial_{x}\left(h \sum_{\beta=1}^{M} l_{\beta} u_{\beta}\right)=0, \\
\partial_{t} m_{\alpha}+\partial_{x} q_{\alpha}=\frac{1}{l_{\alpha}} \sum_{j=0}^{N} \rho_{j} \Delta_{-}^{\alpha} H_{j, \alpha+1 / 2}(\Phi), \\
\partial_{t} q_{\alpha}+\partial_{x}\left(\frac{q_{\alpha}^{2}}{m_{\alpha}}\right)+\partial_{x}\left(g h\left[\frac{1}{2} l_{\alpha} m_{\alpha}+\sum_{\beta=\alpha+1}^{M} l_{\beta} m_{\beta}\right]\right) \\
=\sum_{j=0}^{N} \frac{\rho_{j}}{l_{\alpha}} \Delta_{-}^{\alpha}\left(u_{\alpha+1 / 2} H_{j, \alpha+1 / 2}(\Phi)\right)+\left(p_{\mathrm{S}}+g h \sum_{\beta=\alpha+1}^{M} l_{\beta} \rho_{\beta}(\Phi)\right) \partial_{x} h \\
\quad-g h \rho_{\alpha}\left(\partial_{x} z_{\mathrm{B}}+\sum_{\beta=1}^{\alpha-1} l_{\beta} \partial_{x} h\right) .
\end{array}\right.
$$

For simplicity, for the numerical simulations we set $p_{\mathrm{S}}=0$. In a similar manner as for (3.10), we also write the system (4.2) in a compact form (see Appendix B), namely as

$$
\partial_{t} \widetilde{\boldsymbol{w}}+\partial_{x} \widetilde{\boldsymbol{F}}(\widetilde{\boldsymbol{w}})+\widetilde{\boldsymbol{B}}(\widetilde{\boldsymbol{w}}) \partial_{x} \widetilde{\boldsymbol{w}}=\widetilde{\boldsymbol{S}}(\widetilde{\boldsymbol{w}}) \partial_{x} \widetilde{H}+\widetilde{\boldsymbol{G}}(\widetilde{\boldsymbol{w}}),
$$

where we have introduced the vector of unknowns

$$
\widetilde{\boldsymbol{w}}:=(h, \boldsymbol{m}, \boldsymbol{q})^{\mathrm{T}} \in \mathbb{R}^{2 M+1}
$$

and we have used the notation (4.1) to set $\boldsymbol{m}:=\left(m_{1}, m_{2}, \ldots, m_{M}\right)^{\mathrm{T}} \in \mathbb{R}^{M}$. 
While (3.14) involves an unknown vector $\boldsymbol{w}$ of $(N+1) M+1$ components, (4.3) deals with an unknown vector $\widetilde{\boldsymbol{w}}$ of size $2 M+1$. Since $N \geq 1, \widetilde{\boldsymbol{w}}$ is at most of the same size as $\boldsymbol{w}$. However, the larger the number $N$ of solid species becomes, the smaller is the size of (4.3) compared with (3.14). Thus, solving (4.3) is computationally less expensive than solving (3.14). We therefore propose to solve the original system (3.14) over one time step by solving first (4.3) (Step 1), and then to use an upwinding technique to obtain the variables $r_{j, \alpha}$ (see (4.16) and (4.17)) (Step 2). We now state in detail the two steps of this procedure.

\section{Step 1}

We subdivide the horizontal spatial domain into standard computational cells $I_{i}=\left[x_{i-1 / 2}, x_{i+1 / 2}\right]$, and then apply a finite volume scheme for (4.3), where we exclude the vertical flux contributions by subtracting the term $\widetilde{\boldsymbol{G}}(\widetilde{\boldsymbol{w}})$ in the right-hand side. The resulting system has the form

$$
\widetilde{\boldsymbol{W}}_{t}+\mathcal{A}(\widetilde{\boldsymbol{W}}) \cdot \widetilde{\boldsymbol{W}}_{x}=0
$$

where $\widetilde{\boldsymbol{W}}$ is the concatenated vector $\widetilde{\boldsymbol{W}}:=(\widetilde{\boldsymbol{w}}, \widetilde{H})^{\mathrm{T}} \in \Omega \subset \mathbb{R}^{2(M+1)}$ and

$$
\mathcal{A}(\widetilde{\boldsymbol{W}})=\left[\begin{array}{c|c}
\widetilde{\boldsymbol{A}}(\widetilde{\boldsymbol{w}}) & \widetilde{\boldsymbol{S}}(\widetilde{\boldsymbol{w}}) \\
\hline 0 & 0
\end{array}\right]
$$

where $\widetilde{\boldsymbol{A}}(\widetilde{\boldsymbol{w}})=\widetilde{\boldsymbol{B}}(\widetilde{\boldsymbol{w}})+\widetilde{\boldsymbol{J}}(\widetilde{\boldsymbol{w}})$, being $\widetilde{\boldsymbol{J}}(\widetilde{\boldsymbol{w}})$ the Jacobian matrix of $\widetilde{\boldsymbol{F}}(\widetilde{\boldsymbol{w}})$ (see equations (B.4) and (B.5) in Appendix B).

Solutions of (4.5) may develop discontinuities and, due to the non-divergence form of the equations, the notion of weak solution in the sense of distributions cannot be used. The theory introduced by Dal Maso, LeFloch, and Murat [19] is followed here to define weak solutions. This theory allows one to define the nonconservative product $\mathcal{A}(\widetilde{\boldsymbol{W}}) \cdot \widetilde{\boldsymbol{W}}_{x}$ as a bounded measure provided a family of Lipschitz continuous paths $\Psi:[0,1] \times \Omega \times \Omega \rightarrow \Omega$ is prescribed, which must satisfy certain natural regularity conditions, in particular

$$
\Psi\left(0 ; \widetilde{\boldsymbol{W}}_{\mathrm{L}}, \widetilde{\boldsymbol{W}}_{\mathrm{R}}\right)=\widetilde{\boldsymbol{W}}_{\mathrm{L}}, \quad \Psi\left(1 ; \widetilde{\boldsymbol{W}}_{\mathrm{L}}, \widetilde{\boldsymbol{W}}_{\mathrm{R}}\right)=\widetilde{\boldsymbol{W}}_{\mathrm{R}},
$$

and

$$
\Psi(s ; \widetilde{\boldsymbol{W}}, \widetilde{\boldsymbol{W}})=\widetilde{\boldsymbol{W}} \text { for all } s \in[0,1] .
$$

For example, a family of straight segments can be set:

$$
\Psi\left(s ; \widetilde{\boldsymbol{W}}_{\mathrm{L}}, \widetilde{\boldsymbol{W}}_{\mathrm{R}}\right)=\widetilde{\boldsymbol{W}}_{\mathrm{L}}+s\left(\widetilde{\boldsymbol{W}}_{\mathrm{R}}-\widetilde{\boldsymbol{W}}_{\mathrm{L}}\right), \quad s \in[0,1] .
$$

The numerical schemes considered in this work are based on a decomposition of a generalized Roe matrix for (4.5) defined by Toumi in [38]: given a family of paths $\Psi$, a function $\mathcal{A}_{\Psi}: \Omega \times \Omega \mapsto$ $\mathcal{M}_{2(M+1) \times 2(M+1))}(\mathbb{R})$ is called a Roe linearization if it satisfies the following properties:

- for any $\widetilde{\boldsymbol{W}}_{\mathrm{L}}, \widetilde{\boldsymbol{W}}_{\mathrm{R}} \in \Omega, \mathcal{A}_{\Psi}\left(\widetilde{\boldsymbol{W}}_{\mathrm{L}}, \widetilde{\boldsymbol{W}}_{\mathrm{R}}\right)$ has $2(M+1)$ distinct real eigenvalues,

- for every $\widetilde{\boldsymbol{W}} \in \Omega$,

$$
\mathcal{A}_{\Psi}(\widetilde{\boldsymbol{W}}, \widetilde{\boldsymbol{W}})=\mathcal{A}(\widetilde{\boldsymbol{W}})
$$

- for any $\widetilde{\boldsymbol{W}}_{\mathrm{L}}, \widetilde{\boldsymbol{W}}_{\mathrm{R}} \in \Omega$,

$$
\mathcal{A}_{\Psi}\left(\widetilde{\boldsymbol{W}}_{\mathrm{L}}, \widetilde{\boldsymbol{W}}_{\mathrm{R}}\right) \cdot\left(\widetilde{\boldsymbol{W}}_{\mathrm{R}}-\widetilde{\boldsymbol{W}}_{\mathrm{L}}\right)=\int_{0}^{1} \mathcal{A}\left(\Psi\left(s ; \widetilde{\boldsymbol{W}}_{\mathrm{L}}, \widetilde{\boldsymbol{W}}_{\mathrm{R}}\right)\right) \frac{\partial \Psi}{\partial s}\left(s ; \widetilde{\boldsymbol{W}}_{\mathrm{L}}, \widetilde{\boldsymbol{W}}_{\mathrm{R}}\right) \mathrm{d} s .
$$


Following [30], we consider Roe linearizations for system (4.5) given by

$$
\mathcal{A}_{\Psi}\left(\widetilde{\boldsymbol{W}}_{\mathrm{L}}, \widetilde{\boldsymbol{W}}_{\mathrm{R}}\right)=\left[\begin{array}{c|c}
\widetilde{A}_{\Psi}\left(\widetilde{\boldsymbol{W}}_{\mathrm{L}}, \widetilde{\boldsymbol{W}}_{\mathrm{R}}\right) & \widetilde{S}_{\Psi}\left(\widetilde{\boldsymbol{W}}_{\mathrm{L}}, \widetilde{\boldsymbol{W}}_{\mathrm{R}}\right) \\
\hline 0 & 0
\end{array},\right.
$$

where

$$
A_{\Psi}\left(\widetilde{\boldsymbol{W}}_{\mathrm{L}}, \widetilde{\boldsymbol{W}}_{\mathrm{R}}\right)=\widetilde{\mathcal{J}}\left(\widetilde{\boldsymbol{w}}_{\mathrm{L}}, \widetilde{\boldsymbol{w}}_{\mathrm{R}}\right)+\widetilde{B}_{\Psi}\left(W_{\mathrm{L}}, W_{\mathrm{R}}\right) .
$$

Here, $\mathcal{J}\left(\widetilde{\boldsymbol{w}}_{\mathrm{L}}, \widetilde{\boldsymbol{w}}_{\mathrm{R}}\right)$ is a Roe linearization of the Jacobian of the flux $\widetilde{\boldsymbol{F}}(\widetilde{\boldsymbol{w}})$ in the usual sense:

$$
\widetilde{\mathcal{J}}\left(\widetilde{\boldsymbol{w}}_{\mathrm{L}}, \widetilde{\boldsymbol{w}}_{\mathrm{R}}\right) \cdot\left(\widetilde{\boldsymbol{w}}_{\mathrm{R}}-\widetilde{\boldsymbol{w}}_{\mathrm{L}}\right)=\widetilde{\boldsymbol{F}}\left(\widetilde{\boldsymbol{w}}_{\mathrm{R}}\right)-\widetilde{\boldsymbol{F}}\left(\widetilde{\boldsymbol{w}}_{\mathrm{L}}\right),
$$

where $\widetilde{B}_{\Psi}\left(\widetilde{\boldsymbol{W}}_{\mathrm{L}}, \widetilde{\boldsymbol{W}}_{\mathrm{R}}\right)$ is a matrix satisfying

$$
\widetilde{B}_{\Psi}\left(\widetilde{\boldsymbol{W}}_{\mathrm{L}}, \widetilde{\boldsymbol{W}}_{\mathrm{R}}\right) \cdot\left(\widetilde{\boldsymbol{w}}_{\mathrm{R}}-\widetilde{\boldsymbol{w}}_{\mathrm{L}}\right)=\int_{0}^{1} \widetilde{B}\left(\Psi\left(s ; \widetilde{\boldsymbol{W}}_{\mathrm{L}}, \widetilde{\boldsymbol{W}}_{\mathrm{R}}\right)\right) \frac{\partial \Psi_{\widetilde{\boldsymbol{w}}}}{\partial s}\left(s ; W_{\mathrm{L}}, W_{\mathrm{R}}\right) \mathrm{d} s,
$$

and $\widetilde{S}_{\Psi}\left(\widetilde{\boldsymbol{W}}_{\mathrm{L}}, \widetilde{\boldsymbol{W}}_{\mathrm{R}}\right)$ is a vector satisfying

$$
\widetilde{S}_{\Psi}\left(\widetilde{\boldsymbol{W}}_{\mathrm{L}}, \widetilde{\boldsymbol{W}}_{\mathrm{R}}\right)\left(H_{\mathrm{R}}-H_{\mathrm{L}}\right)=\int_{0}^{1} \widetilde{S}\left(\Psi\left(s ; \widetilde{\boldsymbol{W}}_{\mathrm{L}}, \widetilde{\boldsymbol{W}}_{\mathrm{R}}\right)\right) \frac{\partial \Psi_{H}}{\partial s}\left(s ; \widetilde{\boldsymbol{W}}_{\mathrm{L}}, \widetilde{\boldsymbol{W}}_{\mathrm{R}}\right) \mathrm{d} s .
$$

It can be easily shown that if (4.10)-(4.12) are satisfied, then the matrix defined by (4.8)-(4.9) is a Roe linearization provided it has $2(M+1)$ different real eigenvalues. For the numerical tests that we present in the following section we have not obtained any complex eigenvalue. Nevertheless, we have so far not been able to prove that the system is always hyperbolic.

We consider here path-conservative numerical schemes in the sense defined by Parés in [29]. Applied to the system (4.3), the scheme is of the form

$$
\widetilde{\boldsymbol{w}}_{i}^{n+1 / 2}=\widetilde{\boldsymbol{w}}_{i}^{n}-\frac{\Delta t}{\Delta x}\left(\mathcal{F}_{i+1 / 2}^{n}-\mathcal{F}_{i-1 / 2}^{n}+\frac{1}{2}\left(\mathcal{B}_{i+1 / 2}^{n}+\mathcal{B}_{i-1 / 2}^{n}\right)\right)
$$

along with appropriate initial conditions $\widetilde{\boldsymbol{w}}_{i}^{0}$, and where the expressions $\mathcal{F}_{i+1 / 2}^{n}$ and $\mathcal{B}_{i+1 / 2}^{n}$ are defined as follows:

$$
\begin{aligned}
& \mathcal{F}_{i+1 / 2}^{n}:=\frac{1}{2}\left(\widetilde{\boldsymbol{F}}\left(\widetilde{\boldsymbol{w}}_{i}^{n}\right)+\widetilde{\boldsymbol{F}}\left(\widetilde{\boldsymbol{w}}_{i+1}^{n}\right)\right)-\frac{1}{2} \boldsymbol{Q}_{i+1 / 2}^{n}\left(\widetilde{\boldsymbol{w}}_{i+1}^{n}-\widetilde{\boldsymbol{w}}_{i}^{n}-\boldsymbol{\Lambda}_{i+1 / 2}^{n} \widetilde{\boldsymbol{S}}_{i+1 / 2}^{n}\left(\widetilde{H}_{i+1}^{n}-\widetilde{H}_{i}^{n}\right)\right), \\
& \mathcal{B}_{i+1 / 2}^{n}=\widetilde{B}_{i+1 / 2}^{n}\left(\widetilde{\boldsymbol{w}}_{i+1}^{n}-\widetilde{\boldsymbol{w}}_{i}^{n}\right)-\widetilde{S}_{i+1 / 2}^{n}\left(\widetilde{H}_{i+1}^{n}-\widetilde{H}_{i}^{n}\right),
\end{aligned}
$$

where

$$
\widetilde{B}_{i+1 / 2}^{n}:=\widetilde{B}_{\Psi}\left(\widetilde{\boldsymbol{W}}_{i+1}^{n}, \widetilde{\boldsymbol{W}}_{i}^{n}\right) \text { and } \quad \widetilde{S}_{i+1 / 2}^{n}:=\widetilde{S}_{\Psi}\left(\widetilde{\boldsymbol{W}}_{i+1}^{n}, \widetilde{\boldsymbol{W}}_{i}^{n}\right) .
$$

The matrix $\boldsymbol{\Lambda}_{i+1 / 2}^{n}$ represents an approximation of the inverse of $\widetilde{A}_{i+1 / 2}^{n}=\widetilde{A}_{\Psi}\left(\widetilde{\boldsymbol{W}}_{i+1}^{n}, \widetilde{\boldsymbol{W}}_{i}^{n}\right)$. In addition, $\boldsymbol{Q}_{i+1 / 2}^{n}$ is the numerical viscosity matrix whose definition identifies the particular finite volume method used. For example, the Roe method is defined by $\boldsymbol{Q}_{i+1 / 2}^{n}=\left|\widetilde{\boldsymbol{A}}_{i+1 / 2}^{n}\right|$. An interesting alternative to the Roe method for systems with a great number of unknowns are PVM ("polynomial viscosity matrix") methods (see [15]).

In the sequel, for sake of simplicity, we shall omit the upper index $n$ in the notation of $\mathcal{F}_{i+1 / 2}^{n}$, and then we denote the components of this vector merely by

$$
\mathcal{F}_{i+1 / 2}=\left(\mathcal{F}_{i+1 / 2}^{h}, \mathcal{F}_{i+1 / 2}^{m_{1}}, \ldots, \mathcal{F}_{i+1 / 2}^{m_{M}}, \mathcal{F}_{i+1 / 2}^{q_{1}}, \ldots, \mathcal{F}_{i+1 / 2}^{q_{M}}\right)^{\mathrm{T}} .
$$

On the other hand, if we denote by $\boldsymbol{F}^{r_{j, \alpha}}$ the components of $\boldsymbol{F}$ corresponding to the variables $r_{j, \alpha}$, and by $\widetilde{\boldsymbol{F}}^{m_{\alpha}}$ the components of $\widetilde{\boldsymbol{F}}$ corresponding to the variables $m_{\alpha}$, note that we have the relationship

$$
\boldsymbol{F}^{r_{j, \alpha}}=\frac{r_{j, \alpha}}{m_{\alpha}} \widetilde{\boldsymbol{F}}^{m_{\alpha}}
$$


Moreover, utilizing that the concentration of the different species are passive scalars in the system, we approximate (4.15) by an upwind scheme related to the numerical flux of the total concentration of each species.

Then, from the previous components of the numerical fluxes, we determine those corresponding to the variables $r_{j, \alpha}$ using the following an upwinding formula

$$
\mathcal{F}_{i+1 / 2}^{r_{j, \alpha}}:=\left\{\begin{array}{ll}
\frac{\left(r_{j, \alpha}\right)_{i}}{\left(m_{\alpha}\right)_{i}} \mathcal{F}_{i+1 / 2}^{m_{\alpha}} & \text { if } \mathcal{F}_{i+1 / 2}^{m_{\alpha}}>0, \\
\frac{\left(r_{j, \alpha}\right)_{i+1}}{\left(m_{\alpha}\right)_{i+1}} \mathcal{F}_{i+1 / 2}^{m_{\alpha}} & \text { otherwise, }
\end{array} \quad j=1, \ldots, N,\right.
$$

and once again, with suitable initial data $\left(r_{j, \alpha}\right)_{i}^{0}$, we set the scheme

$$
\left(r_{j, \alpha}\right)_{i}^{n+1 / 2}=\left(r_{j, \alpha}\right)_{i}^{n}-\frac{\Delta t}{\Delta x}\left(\mathcal{F}_{i+1 / 2}^{r_{j, \alpha}}-\mathcal{F}_{i-1 / 2}^{r_{j, \alpha}}+\frac{1}{2}\left(\mathcal{B}_{i+1 / 2}^{r_{j, \alpha}}+\mathcal{B}_{i-1 / 2}^{r_{j, \alpha}}\right)\right) .
$$

The quantity $\mathcal{B}_{i+1 / 2}^{r_{j, \alpha}}$ is defined analogously to (4.14) with the subtracted parts, corresponding to the variables $r_{j, \alpha}$, from the matrix $\boldsymbol{B}(\boldsymbol{w})$ in (3.14). Indeed, there is no action of the vector $\boldsymbol{S}(\boldsymbol{w})$ here (see Appendix A) and we still exclude the contribution of the vertical fluxes incorporated within $\boldsymbol{G}(\boldsymbol{w})$ for the splitting purpose. At this step, we obtain from (4.13) the intermediate solution

$$
\widetilde{\boldsymbol{w}}_{i}^{n+1 / 2}=\left(h_{i}^{n+1 / 2}, \boldsymbol{m}_{i}^{n+1 / 2}, \boldsymbol{q}_{i}^{n+1 / 2}\right)^{\mathrm{T}},
$$

where

$$
\boldsymbol{m}_{i}^{n+1 / 2}=\left(\left(m_{1}\right)_{i}^{n+1 / 2}, \ldots,\left(m_{M}\right)_{i}^{n+1 / 2}\right)^{\mathrm{T}}, \quad \boldsymbol{q}_{i}^{n+1 / 2}=\left(\left(q_{1}\right)_{i}^{n+1 / 2}, \ldots,\left(q_{M}\right)_{i}^{n+1 / 2}\right)^{\mathrm{T}},
$$

and then from (4.17), we get the intermediate solution

$$
\boldsymbol{r}_{i}^{n+1 / 2}=\left(\ldots,\left(r_{1, \alpha}\right)_{i}^{n+1 / 2},\left(r_{2, \alpha}\right)_{i}^{n+1 / 2}, \ldots,\left(r_{N, \alpha}\right)_{i}^{n+1 / 2}, \ldots\right)^{\mathrm{T}} .
$$

\section{Step 2}

We complete the numerical procedure by including the contribution of the numerical vertical fluxes expressed by the matrices $\widetilde{\boldsymbol{G}}(\widetilde{\boldsymbol{w}})$ and $\boldsymbol{G}(\boldsymbol{w})$ in the right-hand sides of (4.3) and (3.14), respectively (see Appendices A and B). These numerical vertical fluxes, namely $f_{j, \alpha+1 / 2}(\Phi)$ for all $j=1, \ldots, N$ and $\alpha=0,1, \ldots, M$, are computed by using the formula of [12, Scheme 8] stated for a single-layer model. Hence, we set

$$
\begin{aligned}
f_{j, \alpha+1 / 2}\left(\Phi_{\alpha}, \Phi_{\alpha+1}\right)= & \frac{1}{2}\left(\phi_{j, \alpha} v_{j}^{\mathrm{MLB}}\left(\Phi_{\alpha}\right)+\phi_{j, \alpha+1} v_{j}^{\mathrm{MLB}}\left(\Phi_{\alpha+1}\right)\right)-\frac{E_{\alpha+1}}{2}\left(\phi_{j, \alpha+1}-\phi_{j, \alpha}\right) \\
& -\frac{\phi_{j, \alpha}}{2}\left|v_{j}^{\mathrm{MLB}}\left(\Phi_{\alpha+1}\right)-v_{j}^{\mathrm{MLB}}\left(\Phi_{\alpha}\right)\right| \operatorname{sgn}\left(\phi_{j, \alpha+1}-\phi_{j, \alpha}\right),
\end{aligned}
$$

where we have used the notation $\Phi_{\alpha}:=\left(\phi_{1, \alpha}, \ldots, \phi_{N, \alpha}\right)^{\mathrm{T}}, E_{\alpha}:=\max _{j=1, \ldots, N}\left|v_{j}^{\mathrm{MLB}}\left(\Phi_{\alpha}\right)\right|$, and the hindered settling velocities $v_{j}^{\mathrm{MLB}}(\Phi)$ are given by $(2.4)$.

Let us denote by $\widetilde{\boldsymbol{G}}_{\boldsymbol{q}}(\widetilde{\boldsymbol{w}})$ the components of $\widetilde{\boldsymbol{G}}(\widetilde{\boldsymbol{w}})$ corresponding to the variables $\boldsymbol{q}$ in (4.3). Analogously, we denote by $\boldsymbol{G}_{\boldsymbol{r}}(\boldsymbol{w})$, the components of $\boldsymbol{G}(\boldsymbol{w})$ corresponding to the variables $\boldsymbol{r}$ in (3.15). Then we set the following updates:

$$
\begin{aligned}
& h_{i}^{n+1}=h_{i}^{n+1 / 2}, \\
& \boldsymbol{q}_{i}^{n+1}=\boldsymbol{q}_{i}^{n+1 / 2}+\Delta t \widetilde{\boldsymbol{G}}_{q}\left(\widetilde{\boldsymbol{w}}_{i}^{n+1 / 2}\right), \\
& \boldsymbol{r}_{i}^{n+1}=\boldsymbol{r}_{i}^{n+1 / 2}+\Delta t \boldsymbol{G}_{r}\left(\boldsymbol{w}_{i}^{n+1 / 2}\right),
\end{aligned}
$$




$$
\begin{aligned}
& \left(\phi_{j, \alpha}\right)_{i}^{n+1}=\frac{\left(r_{j, \alpha}\right)_{i}^{n+1}}{h_{i}^{n+1}}, \quad j=1,2, \ldots, N, \\
& \left(m_{\alpha}\right)_{i}^{n+1}=\rho_{0} h_{i}^{n+1}+\sum_{j=1}^{N}\left(\rho_{j}-\rho_{0}\right)\left(r_{j, \alpha}\right)_{i}^{n+1},
\end{aligned}
$$

where $\boldsymbol{w}_{i}^{n+1 / 2}=\left(h_{i}^{n+1 / 2}, \boldsymbol{q}_{i}^{n+1 / 2}, \boldsymbol{r}_{i}^{n+1 / 2}\right)^{\mathrm{T}}$ as introduced in (3.15). We may also define

$$
\left(r_{0, \alpha}\right)_{i}^{n+1}=h_{i}^{n+1}-\sum_{j=1}^{N}\left(r_{j, \alpha}\right)_{i}^{n+1}, \quad\left(\phi_{0, \alpha}\right)_{i}^{n+1}=1-\sum_{j=1}^{N}\left(\phi_{j, \alpha}\right)_{i}^{n+1},
$$

which represent the pure liquid mass and volumetric concentration, respectively, in layer $\alpha$. Then there follows

$$
\left(m_{\alpha}\right)_{i}^{n+1}=\sum_{j=0}^{N} \rho_{j}\left(\phi_{j, \alpha}\right)_{i}^{n+1} h_{i}^{n+1}
$$

which in the case of equal-density spheres $\rho_{1}=\cdots=\rho_{N}=: \rho_{\mathrm{s}}$ reduces to

$$
\left(m_{\alpha}\right)_{i}^{n+1}=\rho_{\mathrm{s}}\left(1-\left(\phi_{0, \alpha}\right)_{i}^{n+1}\right) .
$$

Recall that $\phi_{j, \alpha}$ for $j=1, \ldots, N$ represents the volumetric concentration of the sediment species $j$ in layer $\alpha$ and one should have $0 \leq \phi_{j, \alpha} \leq 1$ for all $j=0,1, \ldots, N$. To ensure this property, the numerical fluxes are limited.

Finally, let us remark that this method defined in these two steps is deduced as a combination of first order finite volume solvers (see $[12,30]$ ). And the global method is also a first order scheme.

\section{$5 \quad$ Numerical tests}

We use the model developed in the current work to simulate the settling of bidisperse $(N=2)$ suspensions in 2D domains with various bottom geometries. More precisely, we present in Test 1 one example where the bottom has a bump and in Test 2 and 3 two examples with two different kinds of hollow. The parameters are the solid species diameters $d_{1}=4.96 \times 10^{-4} \mathrm{~m}$ and $d_{2}=$ $1.25 \times 10^{-4} \mathrm{~m}$, the density of both solid species is $\rho_{1}=\rho_{2}=2790 \mathrm{~kg} / \mathrm{m}^{3}$, the density of the fluid is $\rho_{0}=1208 \mathrm{~kg} / \mathrm{m}^{3}$, the gravity constant is $g=9.81 \mathrm{~m} / \mathrm{s}^{2}$, and the viscosity of the pure fluid $\mu_{0}=0.02416$ Pa s. (These parameters correspond to experimental data by Schneider et al. [35].) For each horizontal layer $(\alpha=1, \ldots, M)$, the hindrance function $(2.5)$ is used with the exponent $\lambda=4.7$ and a maximum total solid concentration $\phi_{\max }=0.68$. Unless said otherwise, we use SI units in what follows.

\section{Preliminary Test: One-dimensional vertical sedimentation}

First of all, we simulate the settling of a bidisperse sedimentation in a vertical column of height $H=0.3 \mathrm{~m}$ according to the original experiment in [35], which has become a standard example for numerical methods for polydisperse sedimentation (see e.g., [11] and the references cited in that paper). We point out the interaction between the two solid species. This preliminary test will explain some of the forthcoming observations in the $2 \mathrm{D}$ cases. We discretize the height interval with 50 nodes $z_{i}$ and choose the initial solid concentrations $\phi_{1}(t=0)=0.1$ and $\phi_{2}(t=0)=0.05$. Figure 2 displays the simulated concentrations at several times, where we have rescaled height to unity.

In accordance with analytical, experimental and numerical evidence $[7,8,11,13,26,35]$, we observe that the larger species settles more rapidly to the bottom, and forms a sediment layer with a small content of the second (smaller) species only. Most of the smaller species form a second thin layer of sediment which is void of particles of the larger species. The steady state is reached at $t=200 \mathrm{~s}$. 


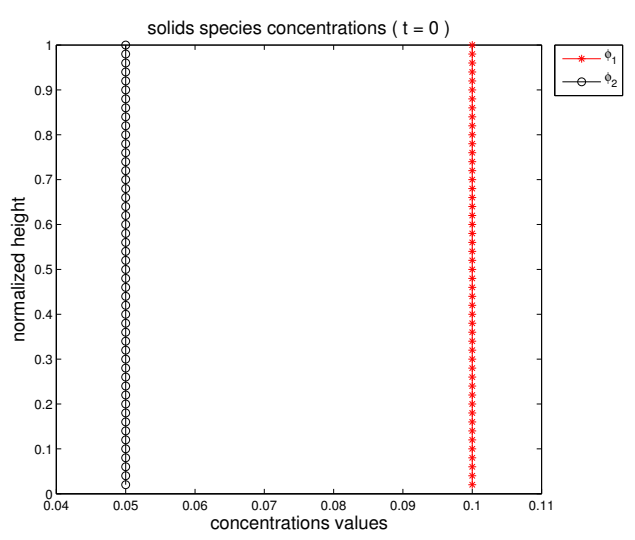

(a) $t=0 \mathrm{~s}$.

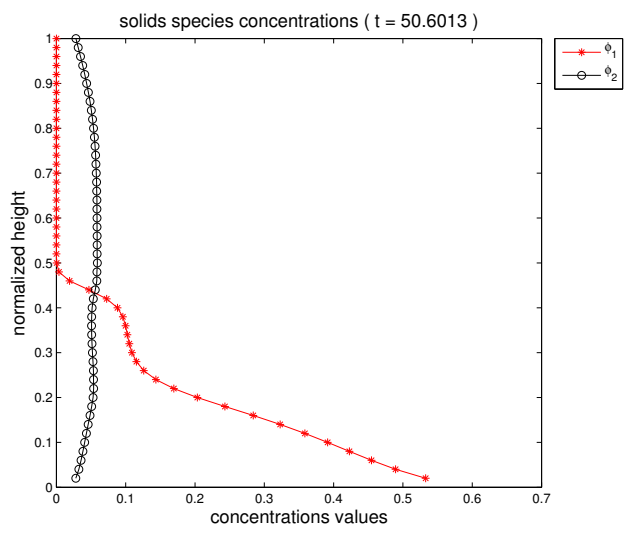

(c) $t=50 \mathrm{~s}$.

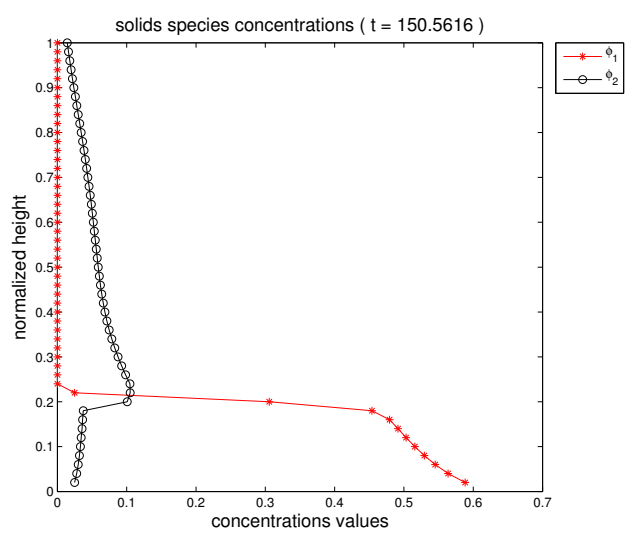

(e) $t=150 \mathrm{~s}$.

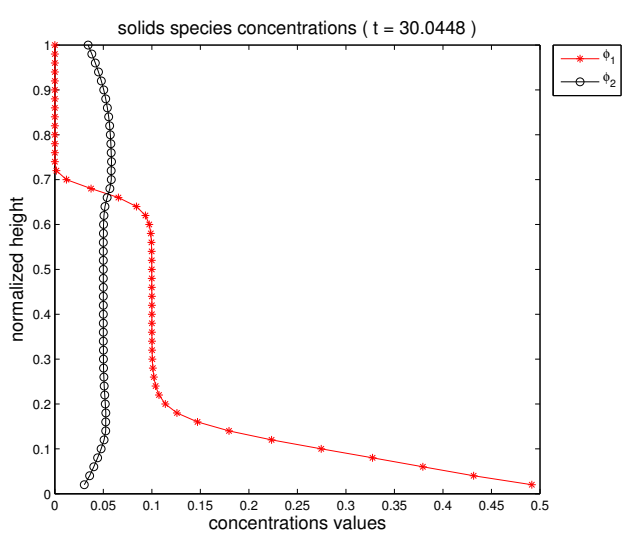

(b) $t=30 \mathrm{~s}$.

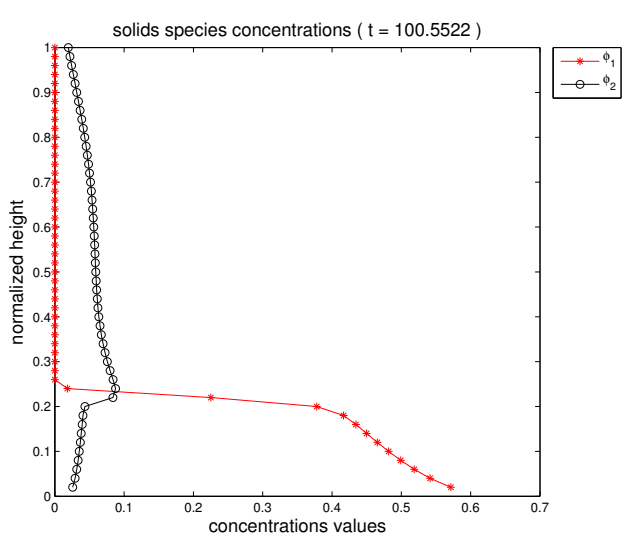

(d) $t=100 \mathrm{~s}$.

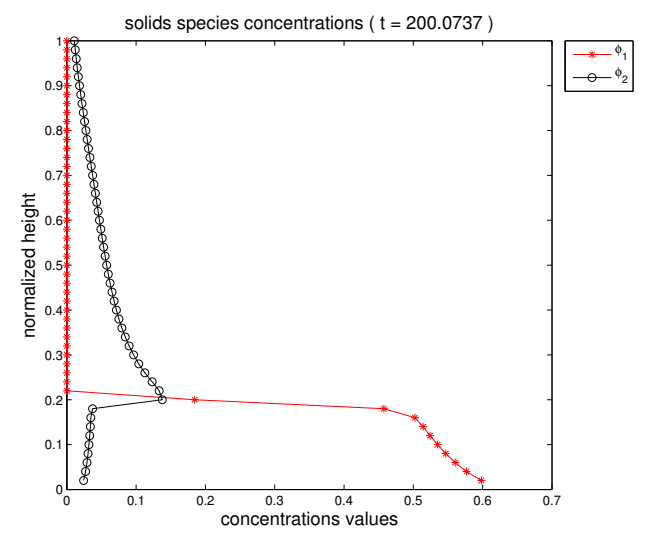

(f) $t=200 \mathrm{~s}$.

Figure 2: Preliminary Test: one-dimensional vertical bidisperse sedimentation: concentrations with respect the height at times $t=0 \mathrm{~s}$ (initial datum) and $t=30,50,100,150,200 \mathrm{~s}$ with $\phi_{1}(t=0)=0.1$ and $\phi_{2}(t=0)=0.05$. 


\subsection{Test 1: Sedimentation in a domain with a bump}

We simulate the sedimentation in a 2D channel of horizontal length $L=1$ which we discretize with 300 nodes $x_{i}$. The vertical direction is discretized using $M=5$ horizontal layers with a CFL number equal to 0.8 . The bottom elevation is given by

$$
z_{\mathrm{B}}(x)=0.2 \exp \left(-40(x-0.5)^{2}\right) .
$$

and we consider the following initial data: $h(t=0)=0.3-z_{\mathrm{B}}$ and

$$
q_{\alpha}(t=0)=0, \quad \phi_{1, \alpha}(t=0)=0.01, \quad \phi_{2, \alpha}(t=0)=0.005 \quad \text { for all } \alpha=1, \ldots, M .
$$

Figures 3-6 report four instants $(t=1,5,15,30 \mathrm{~s})$ of the sedimentation when we consider closed end walls. The closed end walls are simulated by imposing the boundary condition that the velocity vanishes at the two vertical end walls (no-slip boundary condition), i.e.,

$$
\left.u_{\alpha}\right|_{x=0}=\left.u_{\alpha}\right|_{x=L}=0,\left.\quad \varpi_{\alpha}\right|_{x=0}=\left.\varpi_{\alpha}\right|_{x=L}=0 \text { for all } \alpha .
$$

In Figures 3-6, we present the simulated concentrations in each layer by profiles and a colormap, together with the horizontal velocities $u_{\alpha}$ of those layers. Figure 7 represents the velocity vectors $\left(u_{\alpha}, \varpi_{\alpha}\right)$ of the layers for the selected times. The vertical component $\varpi_{\alpha}$ of the velocity vector is computed with a postprocessing technique using the divergence-free condition (2.2) and the kinematic condition at the bottom (see [6]).

Starting from the initial concentration distribution, we notice that the concentration of Species 1 decreases in the upper layers (near the free surface) and increases in the lower ones (near the bottom). This is noticeable in the values of $\phi_{1}$ in the lowest (bottom) and uppermost (free surface) layers (cf. Figure 3 (a)), while in the intermediate layers, $\phi_{1}$ varies very slightly only. In fact, the lowest (respectively, the uppermost) layer carries the highest (respectively, the lowest) value of $\phi_{1}$ all the time along the channel width. However, in the first moments, particles of Species 1 mostly settle just onto the top of the bump and as time evolves, they accumulate symmetrically on the foot of the bump in the lower corners of the domain. In what concerns the second species, we observe a slow symmetric increase in time of the concentration in the bottom layer just on the foot of the bump, whereas above the peak area of the bump, Species 2 is being concentrated quite rapidly in the free surface layer instead. Here again both the lowest and uppermost layers behave very differently from the intermediate ones. Nevertheless, all behave in a symmetric way. The situation described above can be explained by the velocity vector profiles, and in accordance with the interaction between the two solid species as we have seen with the one-dimensional vertical test previously. Indeed, as is illustrated in Figure 7, the bump and the closed end walls induce a circulation of the flow on each side of the bump. The circulation is clockwise to the left and counterclockwise to the right of the bump, and involves strong negative vertical components of the velocity above the bump's peak and along its sides. Hence the flow tries to move the solid particles downward. Therefore, from the interaction issue handled in the preliminary test, the first species accumulates below dripping the bump to fill the lower corners of the domain before and driving the second species away from below. That effect can be visualized better in Figure 6 (a)-(d). Especially in the area above the peak of the bump, there remain positive values of $\phi_{1}$ almost equally distributed in all the layers that prevent the Species 2 from settling into the bottom layer at this area.

Furthermore, we observe the effect of sedimentation on the horizontal velocities. We observe in Figure 7 (a) that these velocities are initially fairly small, and that they increase slowly in time (see Figure 7 (b)-(d)). The reason for this is that we start from the fluid at rest with relatively

low concentrations of solid species. Then the disruption induced by the solid species is not so strong. Next, we observe the combined influence of the bump, the closure of the end walls, and the weight of the suspended solids on the velocities. For instance, Figures $3(\mathrm{e})-6(\mathrm{e})$ for the total 
solid concentration $\phi$ and Figures 3(f)-6(f) for the horizontal velocity $u_{\alpha}$ indicate two types of characteristic behaviour in the velocities. If we focus on the bottom layer (the blue line here), one characteristic behaviour is an increase of velocity near the peak of the bump, whose value reaches zero at the top. This acceleration can be explained by the sliding of the sediments settled on the top of this bump. A second characteristic behaviour is a decrease of velocity from zero on the left of the domain. This is explained by the fall of flow from the top of the bump which pushes the sediment toward the left wall, where its movement is hindered by the accumulation of solids, and is stopped by boundary conditions. As we can see in the figures, the more concentrated the sediment is, the smaller the horizontal velocity becomes in that region. An analogous effect is observed in the right part of the domain.

Figure 8 reports three instants $(t=1,15,30 \mathrm{~s})$ of the sedimentation when we consider an open channel without end walls. The open ends are simulated by duplicating the data across each of the two vertical end walls as transparent boundary conditions. Figure 8 shows the concentrations of each of the solid species at these times. Naturally, profiles turn out similar to the case of closed end walls. However, the significant feature here is that the model renders the expected loss of solids through the boundaries as time evolves. This may be noticed by comparing the values in Figure 8 with those of Figures 3-6.

\subsection{Test 2: Sedimentation in a domain with a hollow}

We simulate now the sedimentation keeping the same 2D channel with the same discretization features and initial conditions that in previous test, but we change the bottom geometry by setting

$$
z_{\mathrm{B}}(x)=0.2\left(1-\exp \left(-40(x-0.5)^{2}\right)\right) .
$$

Figures 9-12 report four instants $(t=1,5,15,30 \mathrm{~s})$ of the sedimentation when we consider closed end walls. In Figures 9-12, we represent the values and colormap of the concentrations in each layer together with the horizontal velocities of those layers. Figure 13 represents the velocity vectors of the layers for the selected times.

From the initial concentrations distribution, we notice a rapid and a massive movement of Species 1 from the upper layers the lower ones. We observe that the lowest layer differs from the others by the accumulation of particles on the bottom of the hollow. As in Test 1, the level of concentration in the intermediate layers varies very slightly. Actually, in the first moments, the particles mostly settle outside the hollow just onto the top of each side and as time evolves, they accumulate symmetrically inside. Species 2 immediately accumulates on the bottom inside the hollow, and its concentration increases in time. Hence, the hollow appears to constitute a particle trap. Moreover, the species also accumulate quite quickly in the free surface layer in the upper corners of the domain.

The phenomena we have just described can be explained once more looking at the velocity vectors profiles and according to the interaction between the two solid species. As illustrated in Figure 13, the hollow and the closed end walls induce two opposite circulations in the flow separated by the vertical axis of symmetry axis at $x=0.5 \mathrm{~m}$. The circulation to the left is counterclockwise and that to the right is clockwise. Hence inside the hollow, the flow tries to move the solid species downward from each side whereas in the middle the moving is upward. Therefore from the interaction issue, Species 1 accumulates below dripping both sides of the hollow to concentrate essentially inside. That effect is better visualized in Figure 12. In addition, under the same flow regime, the hollow traps a portion of Species 2, while the other part is sent to the corners above by the circulation. Next, there remain some positive concentrations of Species 1 in all the layers in these regions, with a minor value in the free surface layer than in the two previous ones. Then, the concentration of Species 2 moved upwards by the circulation is kept in the free surface layer at the corners trying to occupy the place left by Species 1 . 


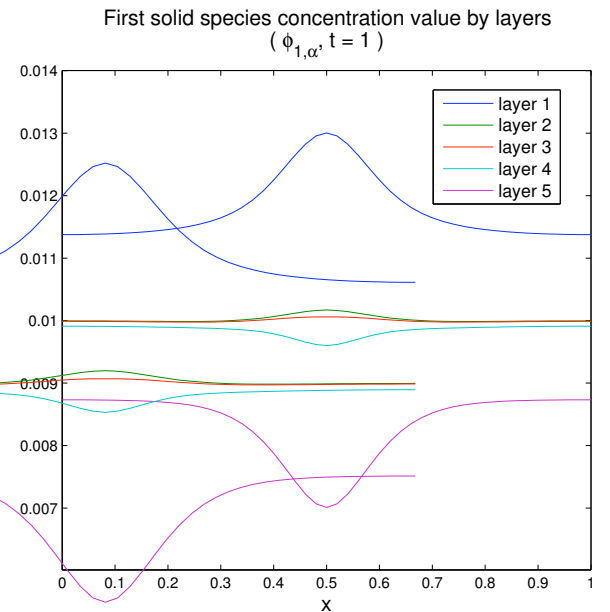

(a) $\phi_{1, \alpha}(t=1 \mathrm{~s})$.

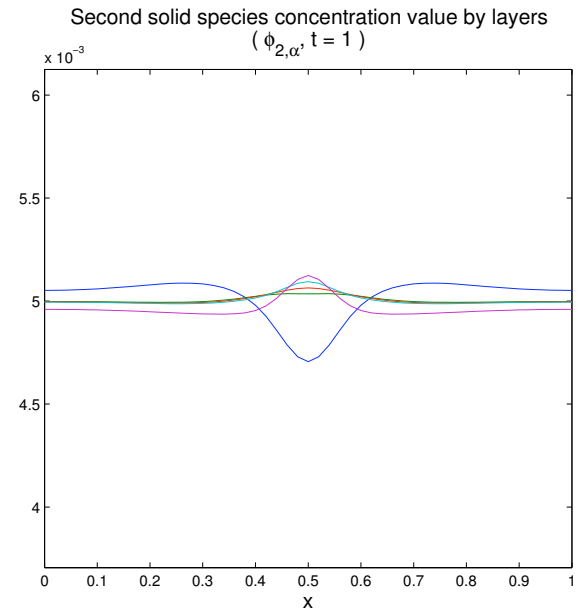

(c) $\phi_{2, \alpha}(t=1 \mathrm{~s})$.

Cumulative solids species concentration value by layers

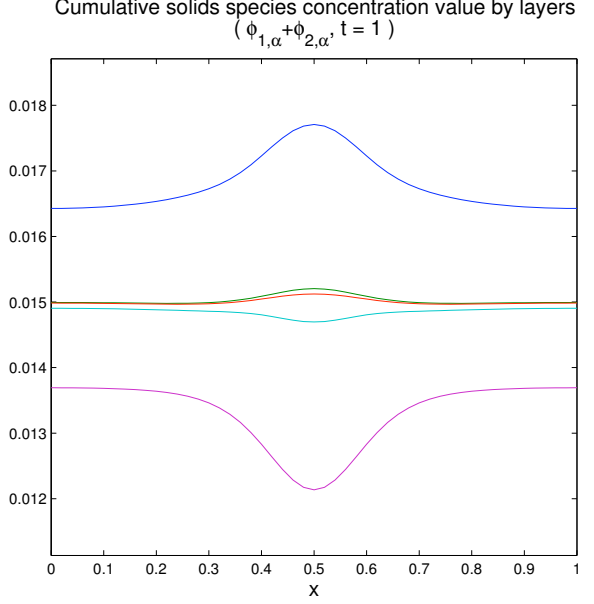

(e) $\left(\phi_{1, \alpha}+\phi_{2, \alpha}\right)(t=1 \mathrm{~s})$.

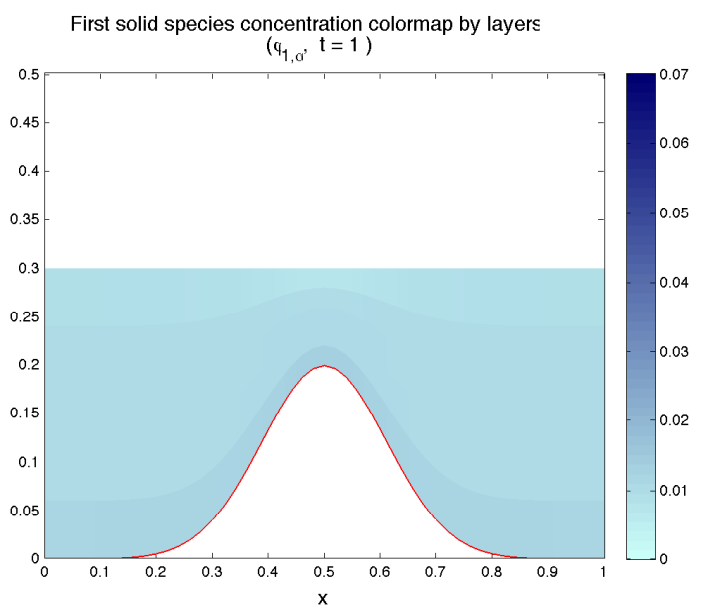

(b) $\phi_{1, \alpha}(t=1 \mathrm{~s})$.

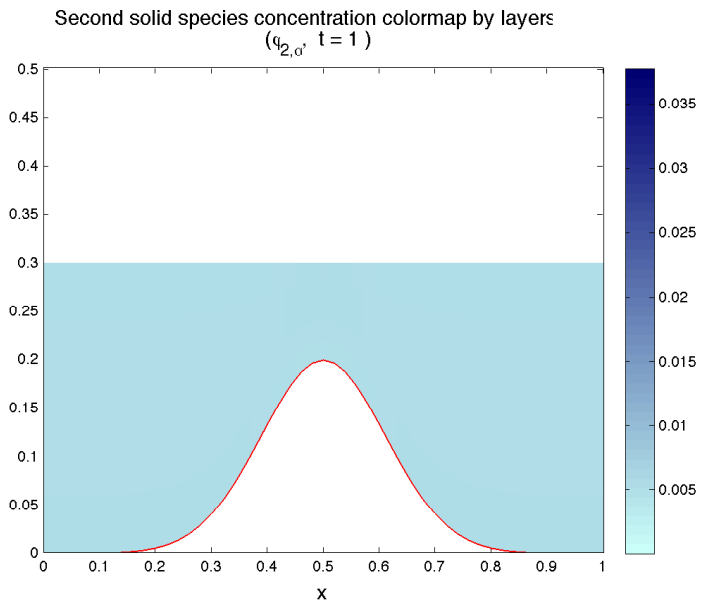

(d) $\phi_{2, \alpha}(t=1 \mathrm{~s})$.

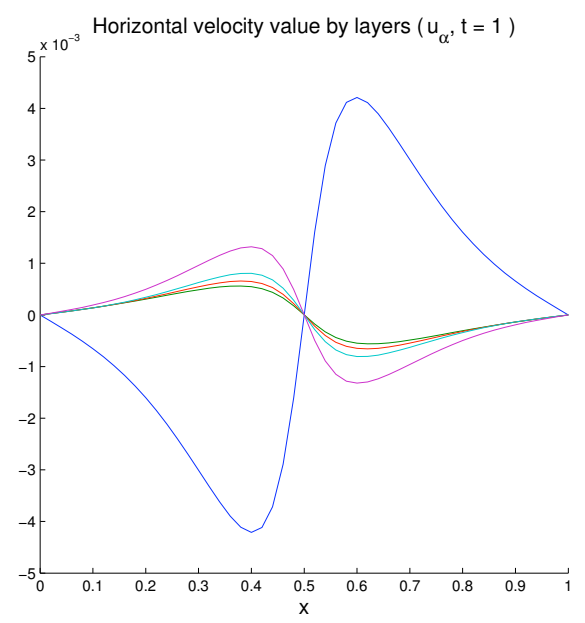

(f) $u_{\alpha}(t=1 \mathrm{~s})$.

Figure 3: Test 1: solid species concentrations and horizontal velocities by layers at time $t=1 \mathrm{~s}$, with closed end walls. 


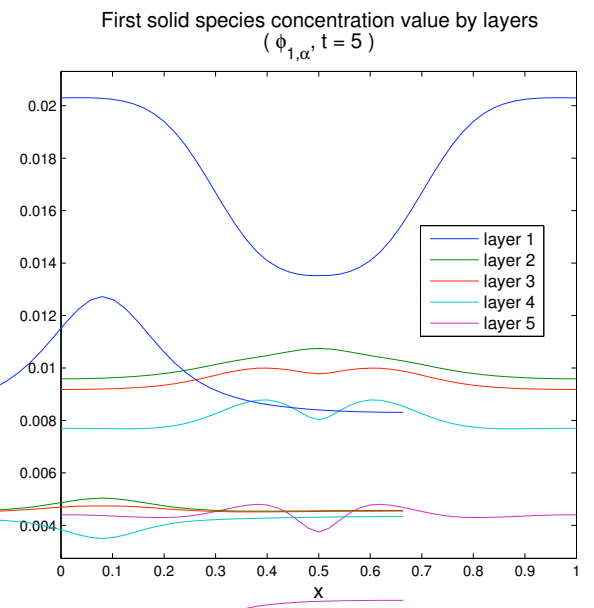

(a) $\phi_{1, \alpha}(t=5 \mathrm{~s})$.

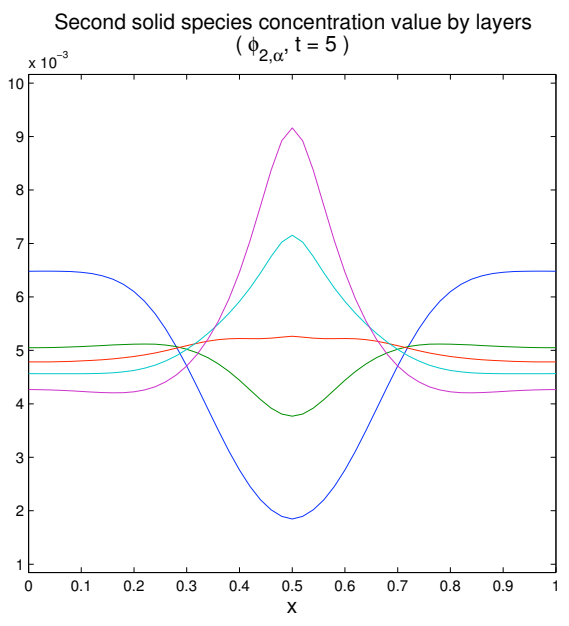

(c) $\phi_{2, \alpha}(t=5 \mathrm{~s})$.

Cumulative solids species concentration value by layers $\left(\phi_{1, \alpha}+\phi_{2, \alpha}, t=5\right)$

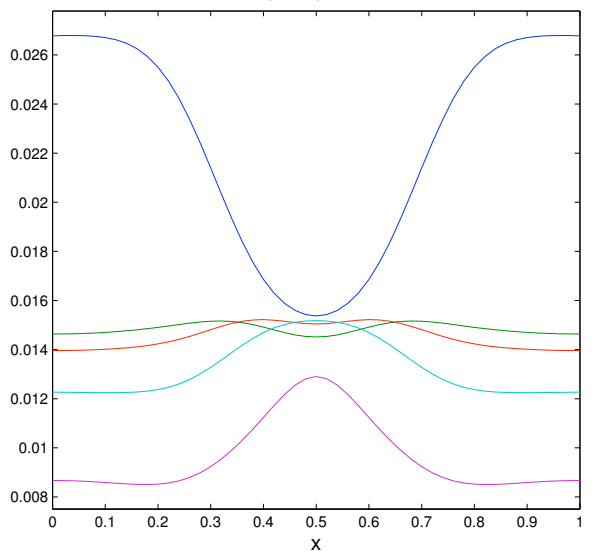

(e) $\left(\phi_{1, \alpha}+\phi_{2, \alpha}\right)(t=5 \mathrm{~s})$.

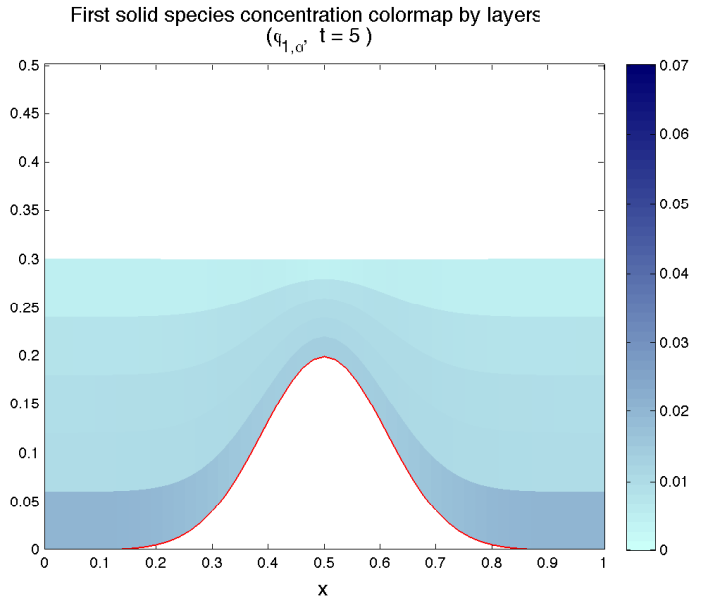

(b) $\phi_{1, \alpha}(t=5 \mathrm{~s})$.

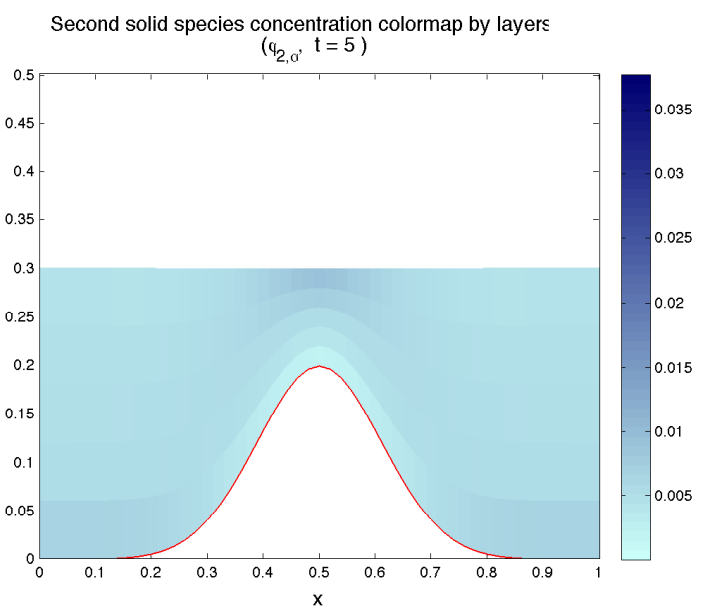

(d) $\phi_{2, \alpha}(t=5 \mathrm{~s})$.

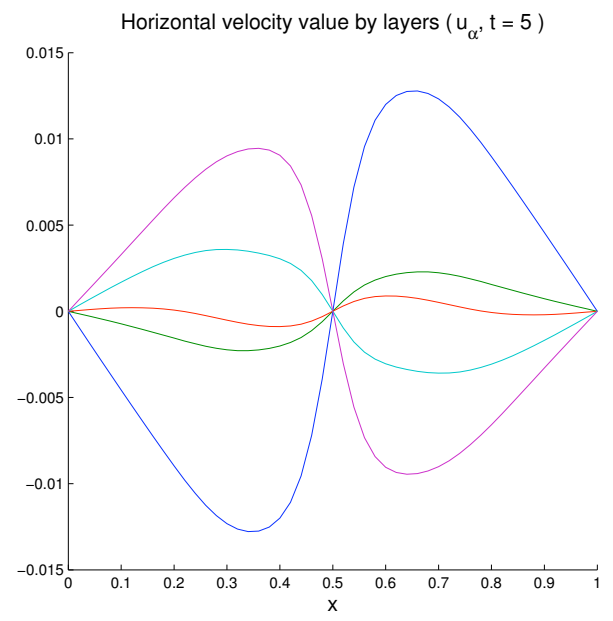

(f) $u_{\alpha}(t=5 \mathrm{~s})$.

Figure 4: Test 1: solid species concentrations and horizontal velocities by layers at time $t=5 \mathrm{~s}$, with closed end walls. 


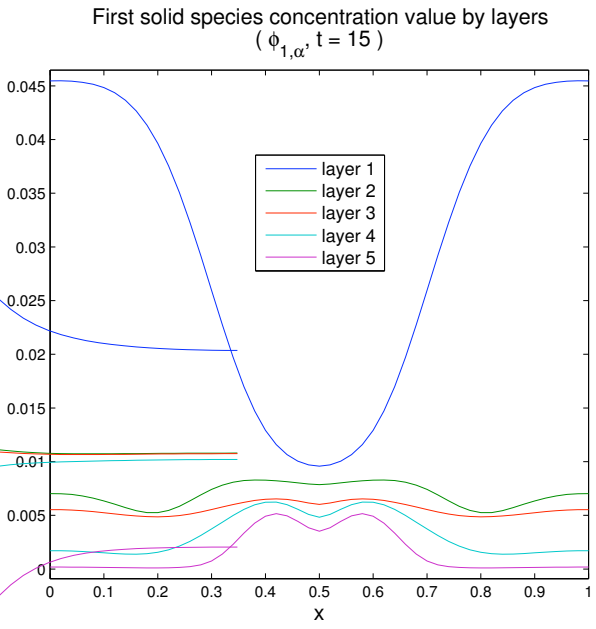

(a) $\phi_{1, \alpha}(t=15 \mathrm{~s})$.

Second solid species concentration value by layers

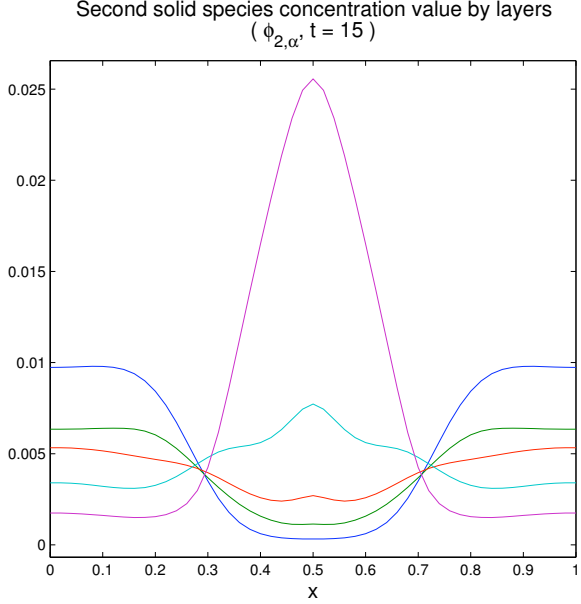

(c) $\phi_{2, \alpha}(t=15 \mathrm{~s})$.

Cumulative solids species concentration value by layers

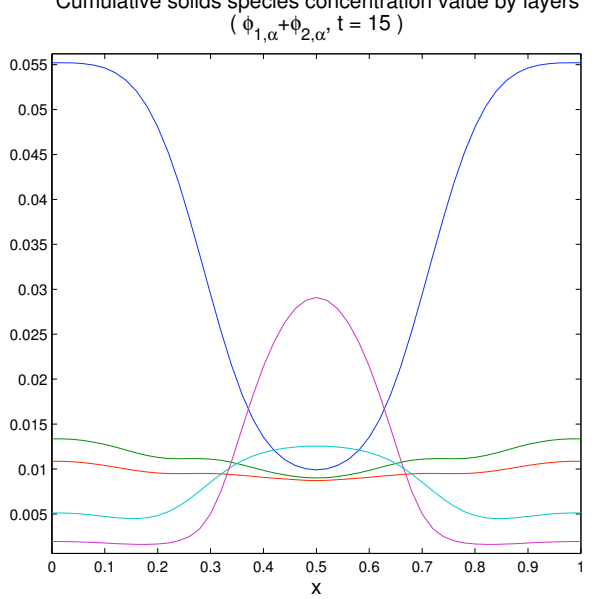

(e) $\left(\phi_{1, \alpha}+\phi_{2, \alpha}\right)(t=15 \mathrm{~s})$.

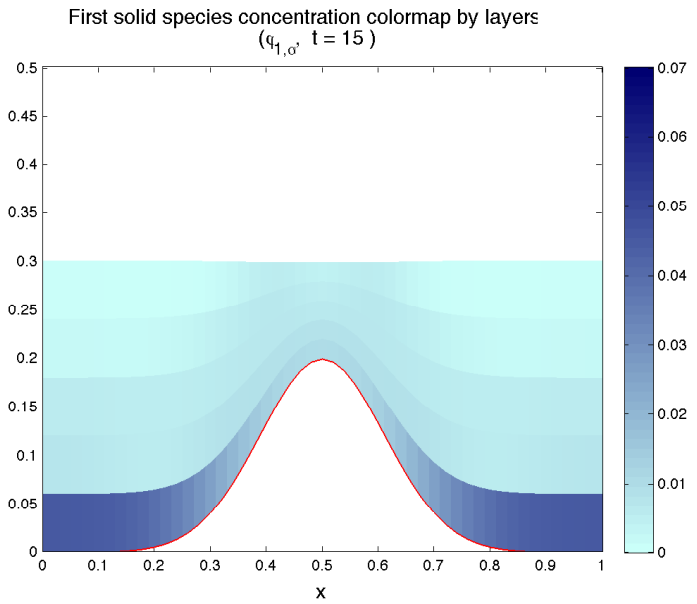

(b) $\phi_{1, \alpha}(t=15 \mathrm{~s})$.

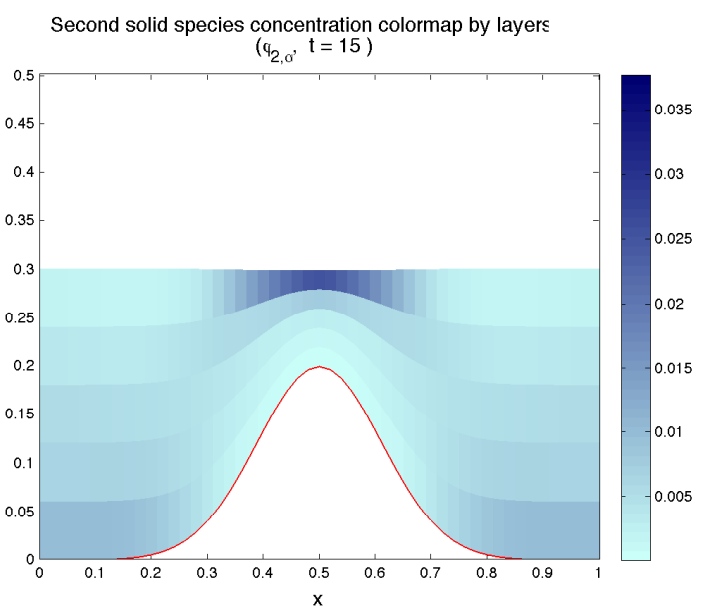

(d) $\phi_{2, \alpha}(t=15 \mathrm{~s})$.

Horizontal velocity value by layers $\left(u_{\alpha}, t=15\right)$

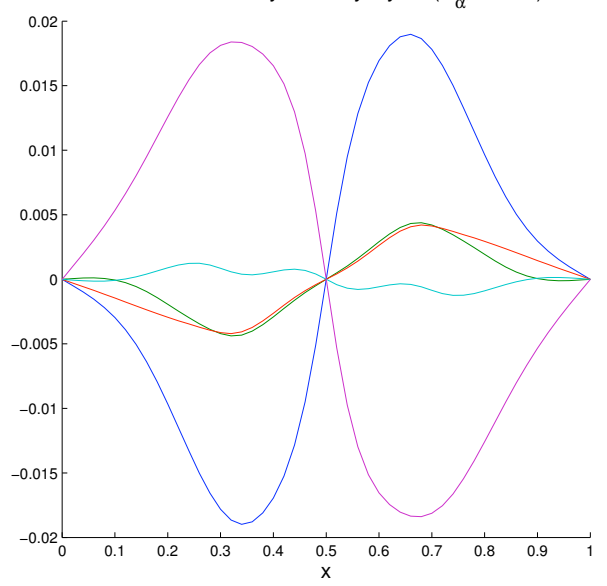

(f) $u_{\alpha}(t=15 \mathrm{~s})$

Figure 5: Test 1: solid species concentrations and horizontal velocities by layers at time $t=15 \mathrm{~s}$, with closed end walls. 


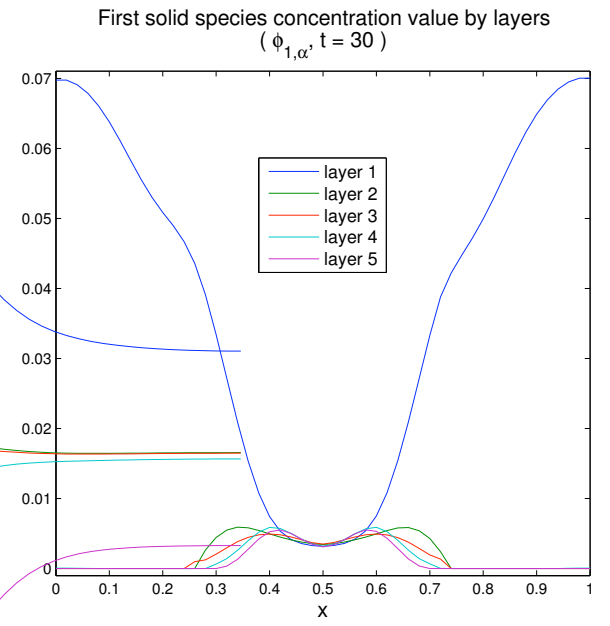

(a) $\phi_{1, \alpha}(t=30 \mathrm{~s})$.

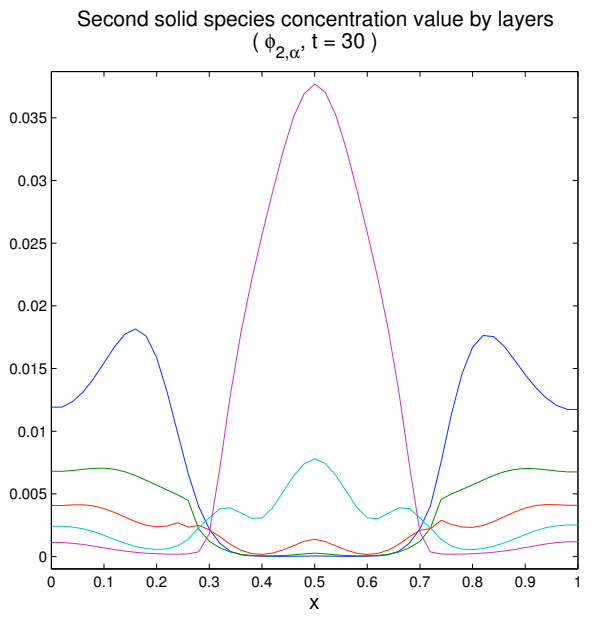

(c) $\phi_{2, \alpha}(t=30 \mathrm{~s})$.

Cumulative solids species concentration value by layers

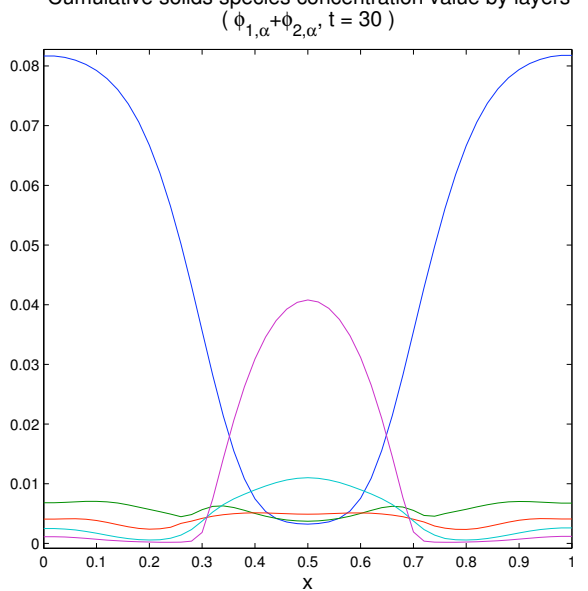

(e) $\left(\phi_{1, \alpha}+\phi_{2, \alpha}\right)(t=30 \mathrm{~s})$.

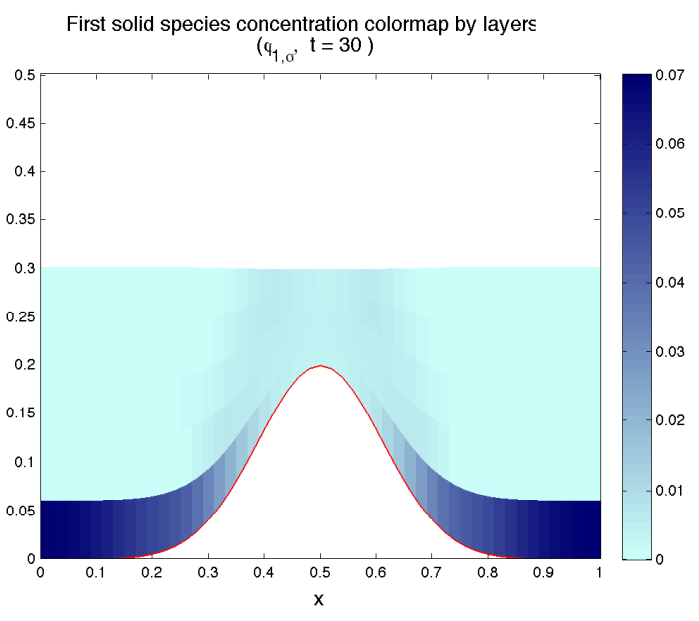

(b) $\phi_{1, \alpha}(t=30 \mathrm{~s})$.

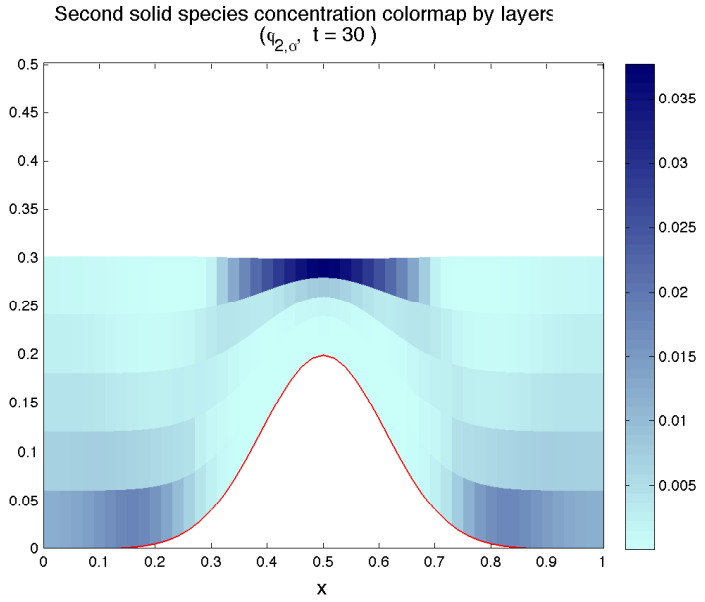

(d) $\phi_{2, \alpha}(t=30 \mathrm{~s})$.

Horizontal velocity value by layers $\left(u_{\alpha}, t=30\right)$

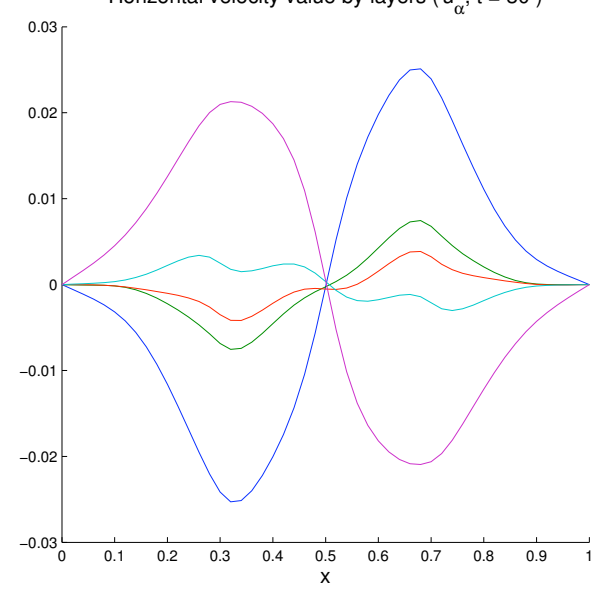

(f) $u_{\alpha}(t=30 \mathrm{~s})$

Figure 6: Test 1: solid species concentrations and horizontal velocities by layers at time $t=30 \mathrm{~s}$, with closed end walls. 


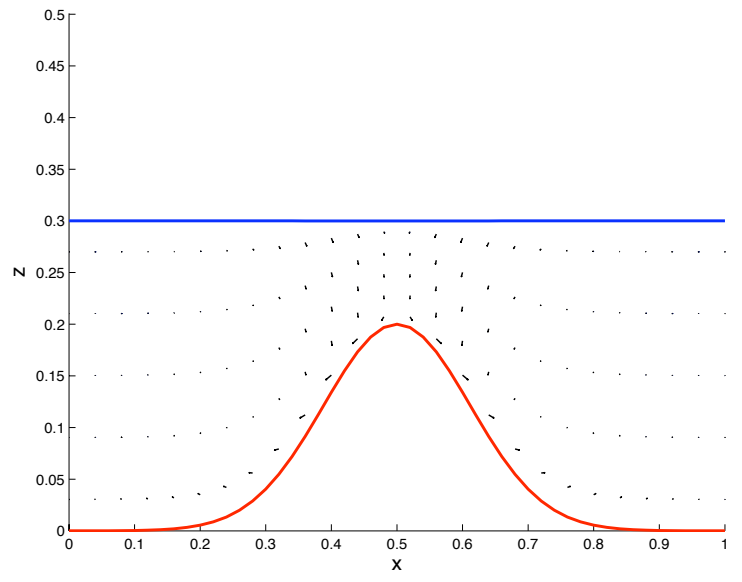

(a) $t=1 \mathrm{~s}$.

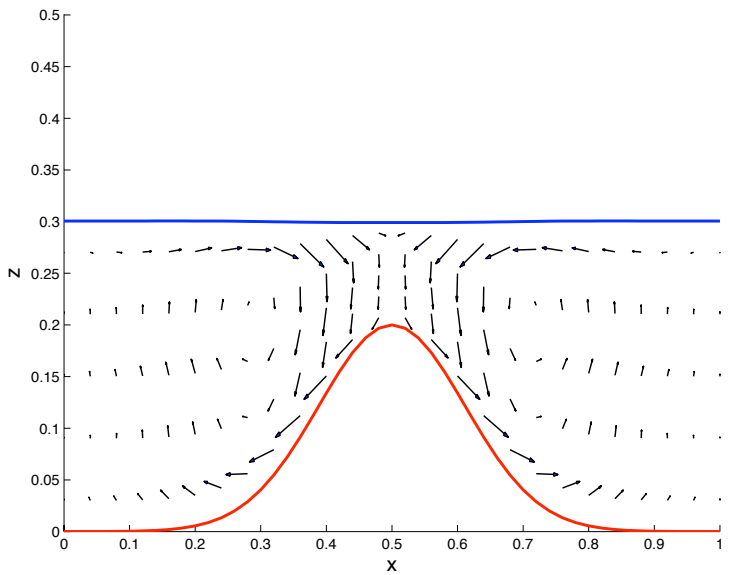

(c) $t=15 \mathrm{~s}$.

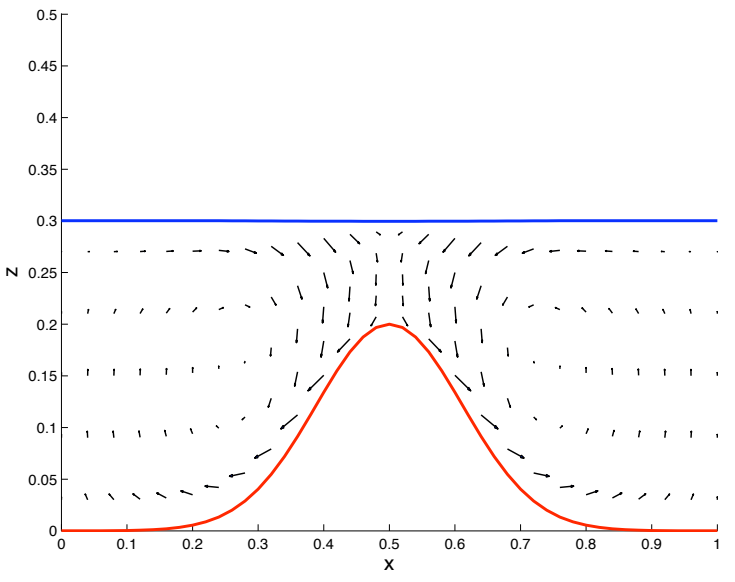

(b) $t=5 \mathrm{~s}$.

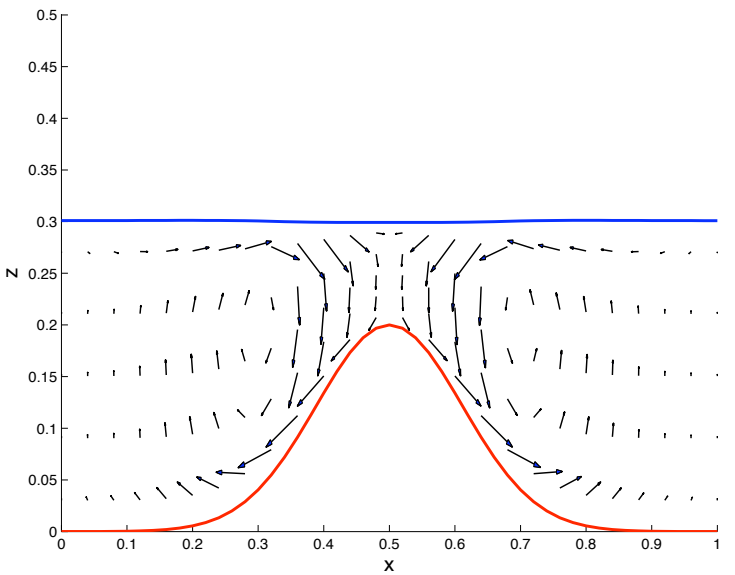

(d) $t=30 \mathrm{~s}$.

Figure 7: Test 1: velocity vectors by layers at the times $t=1,5,15,30 \mathrm{~s}$, with closed end walls (vector lengths multiplied by 2 ). 


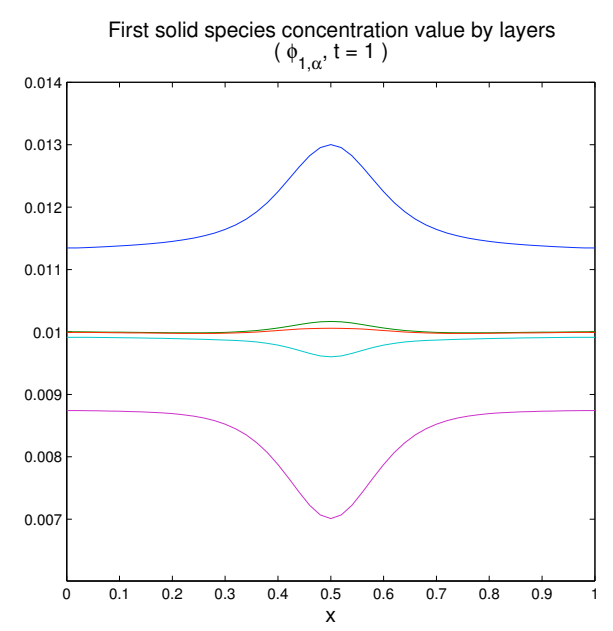

(a) $\phi_{1, \alpha}(t=1 \mathrm{~s})$.

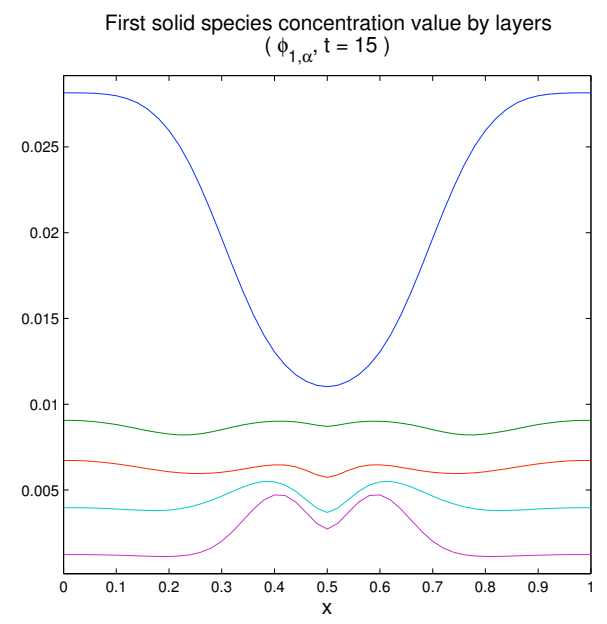

(c) $\phi_{1, \alpha}(t=15 \mathrm{~s})$.

First solid species concentration value by layers

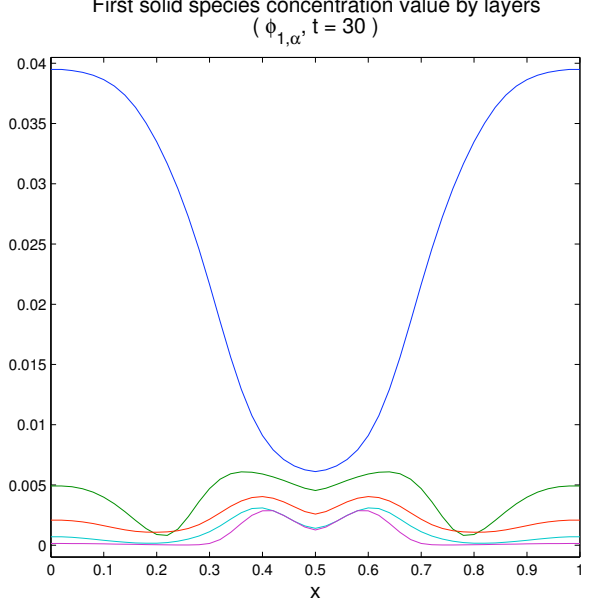

(e) $\phi_{1, \alpha}(t=30 \mathrm{~s})$.

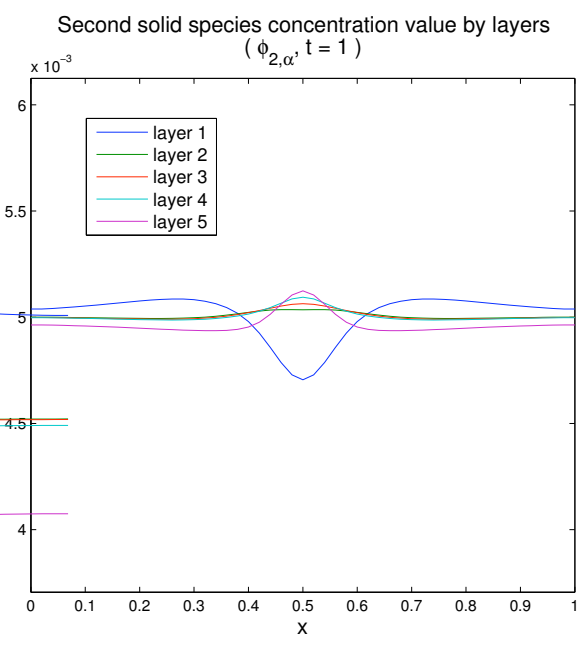

(b) $\phi_{2, \alpha}(t=1 \mathrm{~s})$.

Second solid species concentration value by layers $\left(\phi_{2, \alpha}, \mathrm{t}=15\right)$

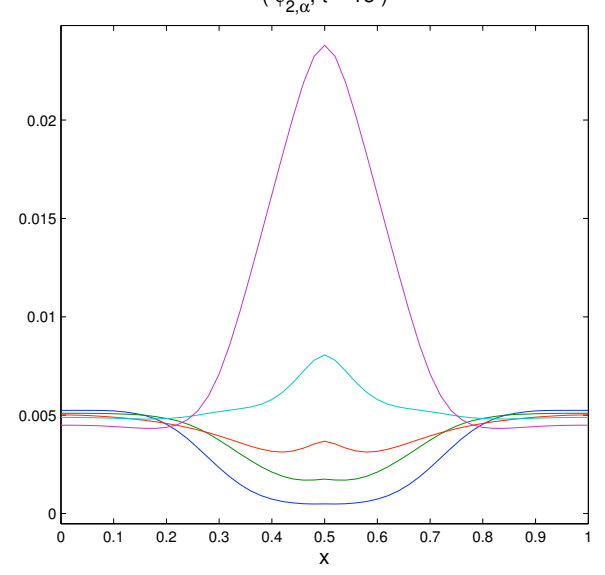

(d) $\phi_{2, \alpha}(t=15 \mathrm{~s})$.

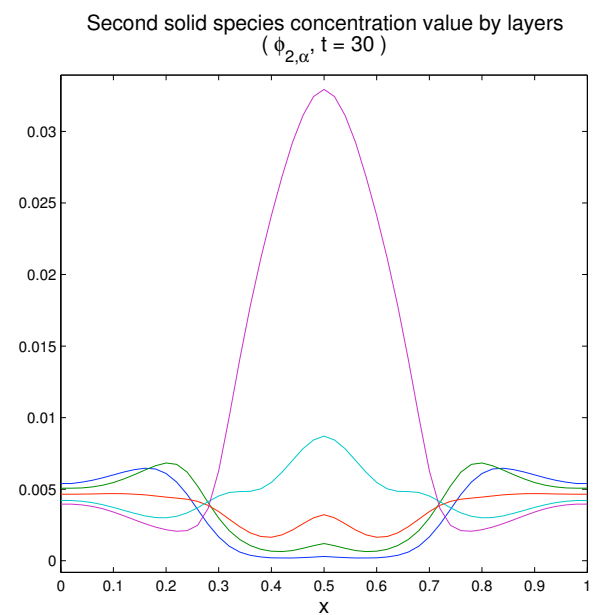

(f) $\phi_{2, \alpha}(t=30 \mathrm{~s})$.

Figure 8: Test 1: solid species concentrations by layers at times $t=1,15,30 \mathrm{~s}$, with open end walls. 
We observe again the effect of the sedimentation on the horizontal velocities. That effect is a combination of the influence of the hollow, the closure of the end walls and the weight in sediments on the velocities. Figures 9(e)-12(e) for the total solid concentrations and Figures 9(f) - 12(f) for the horizontal velocities again indicate two types of characteristic behaviour in the velocities. If we focus on the bottom layer, then one characteristic behaviour is a decreasing velocity value near the base of the hollow. This deceleration may be explained by the hindrance caused by the opposite slipping of the sediment settled on each slope of the hollow and going downward. The second characteristic behaviour is an increase of the velocity value from zero on the left of the domain. Starting with a layer stopped by boundary conditions, that is explained by the fall of flow inside the hollow filtering and getting the layer lighter in this area from the left to the right. As we can see in the figures, the lighter are the sediments the more is the horizontal velocity in that region.

\subsection{Test 3: Imposed velocity and sediment concentration as boundary condition}

In this test we change the form of the hollow and the boundary conditions. More precisely, we spread out the base of the hollow and employ open end boundary conditions along with a fixed horizontal velocity and fixed solid concentrations imposed at the left boundary.

We consider the same discretization features and initial conditions as in Tests 1 and 2. The bottom function $z_{\mathrm{B}}(x)$ is defined by

$$
z_{\mathrm{B}}(x)= \begin{cases}0 & \text { if }\left|x-\frac{1}{2}\right| \leq \frac{1}{8}, \\ p_{2}\left(\left|x-\frac{1}{2}\right|\right) & \text { if } \frac{1}{8} \leq\left|x-\frac{1}{2}\right| \leq \frac{1}{4}, \\ 0.2 & \text { if }\left|x-\frac{1}{2}\right| \geq \frac{1}{4},\end{cases}
$$

where $p_{2}(x)$ is the polynomial of degree two such that $p_{2}\left(\frac{1}{8}\right)=0, p_{2}\left(\frac{1}{4}\right)=0.2$ and $p_{2}^{\prime}\left(\frac{1}{8}\right)=0$. In what follows, we denote by $u_{\mathrm{b}, \alpha}$ the given left boundary velocity, and we impose the left boundary concentrations $\phi_{1, \mathrm{~b}, \alpha}=0.01$ and $\phi_{2, \mathrm{~b}, \alpha}=0.005$. This test aims at studying the effect of the given left boundary velocity on the sedimentation. Figures 14 and 15 report the concentrations of Species 1 and horizontal velocities at times $t=5,30 \mathrm{~s}$ with the respective imposed velocities $u_{\mathrm{b}, \alpha}=0.3 \mathrm{~ms}^{-1}$ and $u_{\mathrm{b}, \alpha}=0.5 \mathrm{~ms}^{-1}$. Figures 16 and 17 report the concentrations of the second species at the times $t=5 \mathrm{~s}$ and $t=30 \mathrm{~s}$ when $u_{\mathrm{b}, \alpha}=0.3 \mathrm{~ms}^{-1}$ and $u_{\mathrm{b}, \alpha}=0.5 \mathrm{~ms}^{-1}$, respectively. For Species 1, we notice a similar initial effect with the two values of $u_{\mathrm{b}, \alpha}$. That is the convection of the sediment toward the right inducing an asymmetric form in layers with an accumulation in the bottom layer, as shown in Figures 14(a) and 15(a), or equivalently in Figures 14(b) and 14(b). As the time evolves, there appears a great difference in the behaviour of both solutions with the two values of $u_{\mathrm{b}, \alpha}$. With the low velocity value, the particles accumulates almost symmetrically in the bottom inside the hollow with an increased concentration (see Figure 14(c) or 14(d)). However, the sedimentation with $u_{\mathrm{b}, \alpha}=0.5 \mathrm{~ms}^{-1}$ keeps the same asymmetric form as the beginning with a non-increased concentration despite the constant supply of particles through the entry boundary (Figure 15(c) or 15(d)). A similar behaviour is observed by Species 2 up to the interaction, already examined earlier, with Species 1, as illustrated in Figures 16 and 17. The previous events reveal clearly that the left boundary velocity $u_{b, \alpha}$ sweeps the sediments along the domain. However with the low velocity value experimented here $\left(u_{b, \alpha}=0.3 \mathrm{~ms}^{-1}\right)$, a major quantity of the provided sediments may stay in the domain dropping and accumulating in the bottom inside the hollow, while only a small quantity stays with the high velocity value $\left(u_{b, \alpha}=0.5 \mathrm{~ms}^{-1}\right)$. This velocity is high enough to carry a significant sediment concentration away from the domain trough the open right end wall. 


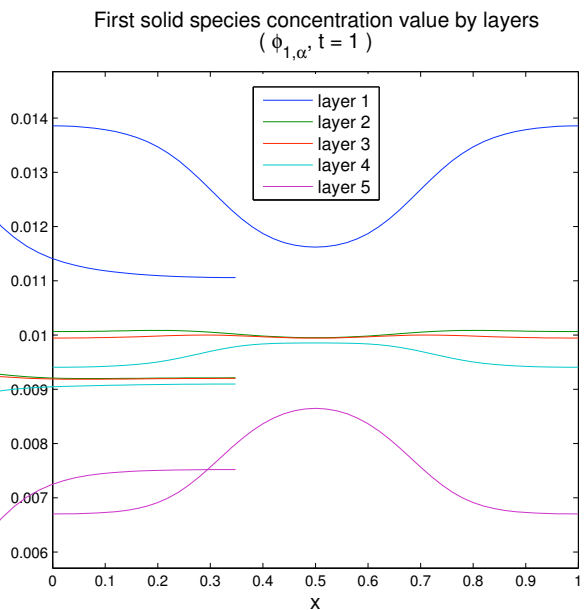

(a) $\phi_{1, \alpha}(t=1 \mathrm{~s})$.

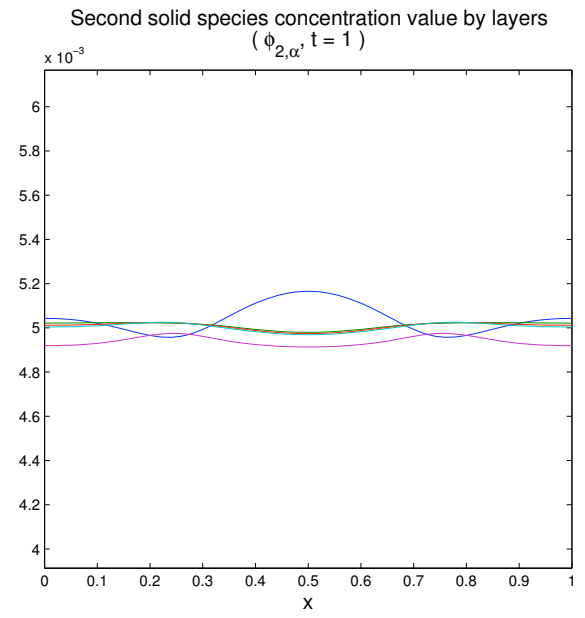

(c) $\phi_{2, \alpha}(t=1 \mathrm{~s})$.

Cumulative solids species concentration value by layers $\left(\phi_{1, \alpha}+\phi_{2, \alpha}, t=1\right)$

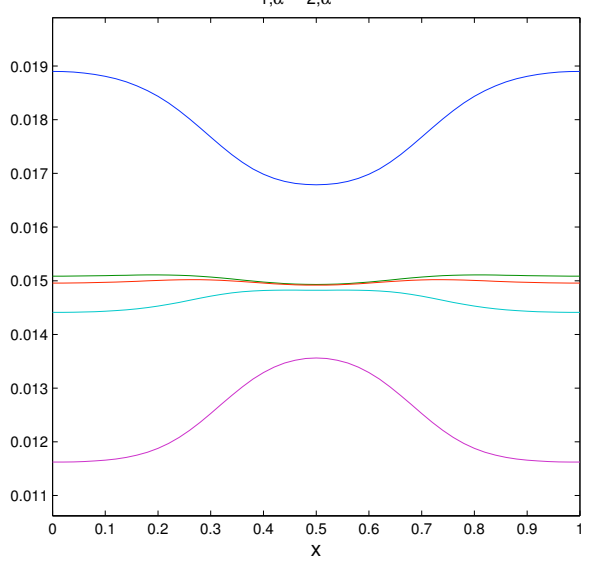

(e) $\left(\phi_{1, \alpha}+\phi_{2, \alpha}\right)(t=1 \mathrm{~s})$.

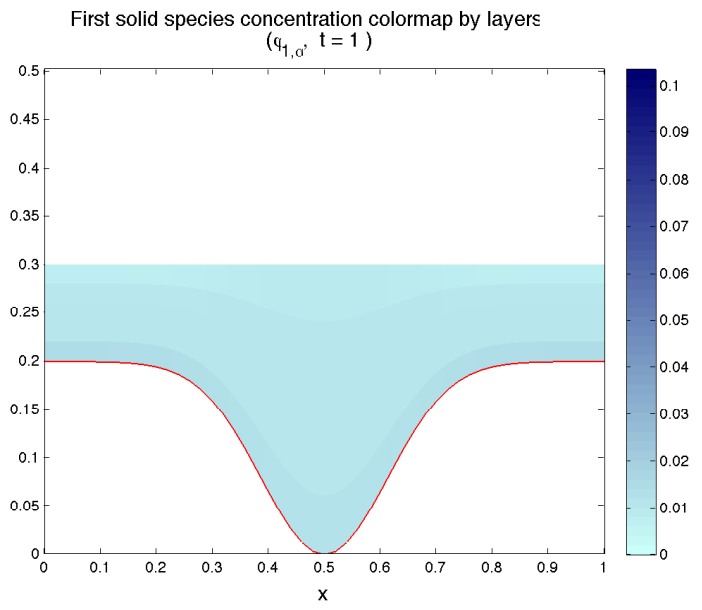

(b) $\phi_{1, \alpha}(t=1 \mathrm{~s})$.

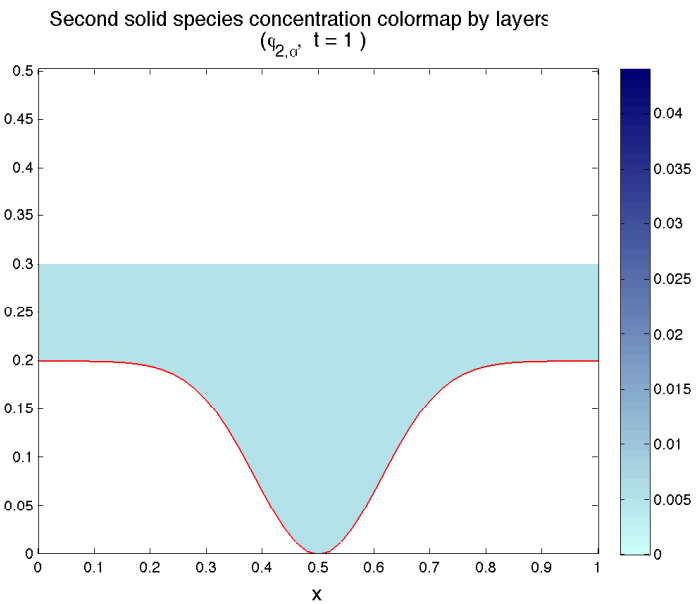

(d) $\phi_{2, \alpha}(t=1 \mathrm{~s})$.

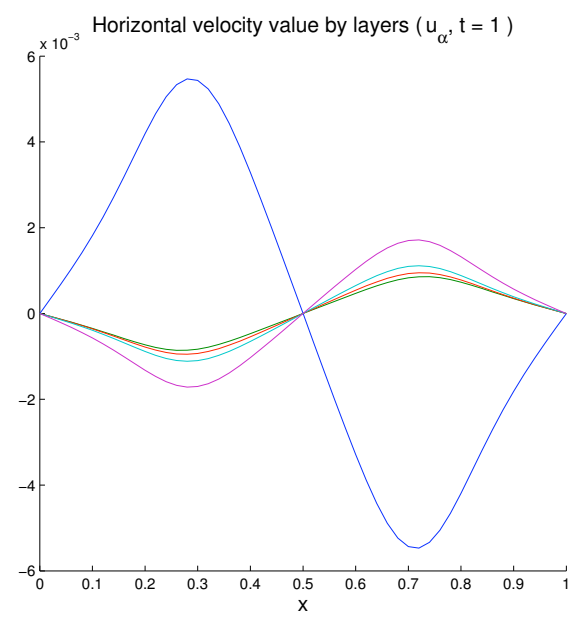

(f) $u_{\alpha}(t=1 \mathrm{~s})$.

Figure 9: Test 2: solid species concentrations and horizontal velocities by layers at the time $t=1$ $\mathrm{s}$, with closed end walls. 


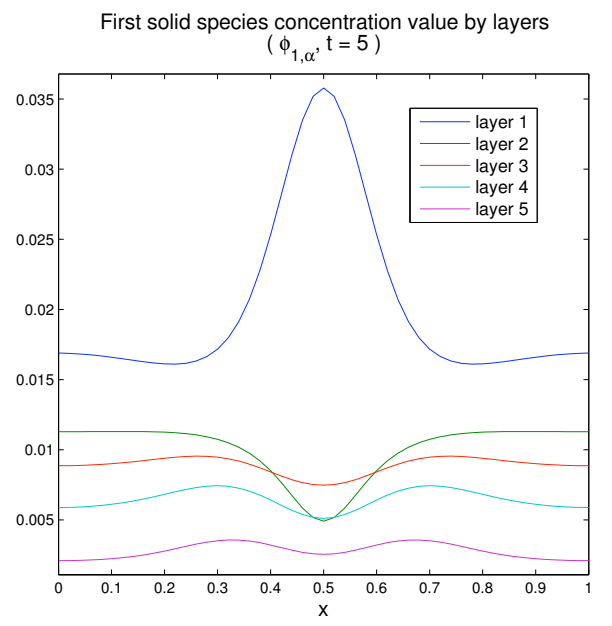

(a) $\phi_{1, \alpha}(t=5 \mathrm{~s})$.

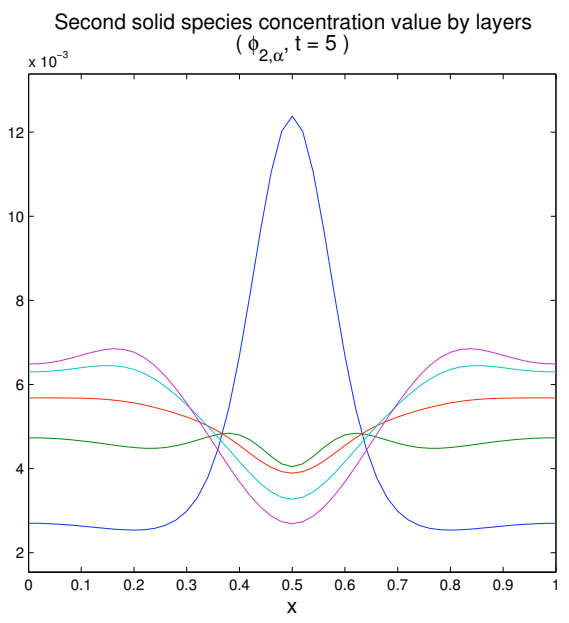

(c) $\phi_{2, \alpha}(t=5 \mathrm{~s})$.

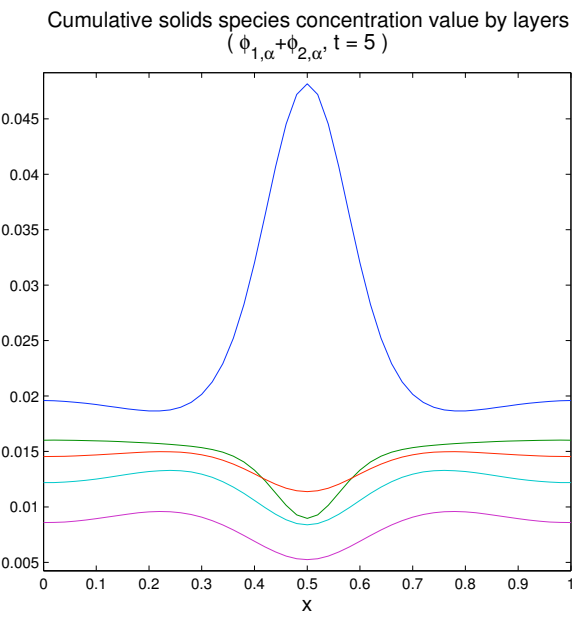

(e) $\left(\phi_{1, \alpha}+\phi_{2, \alpha}\right)(t=5 \mathrm{~s})$.

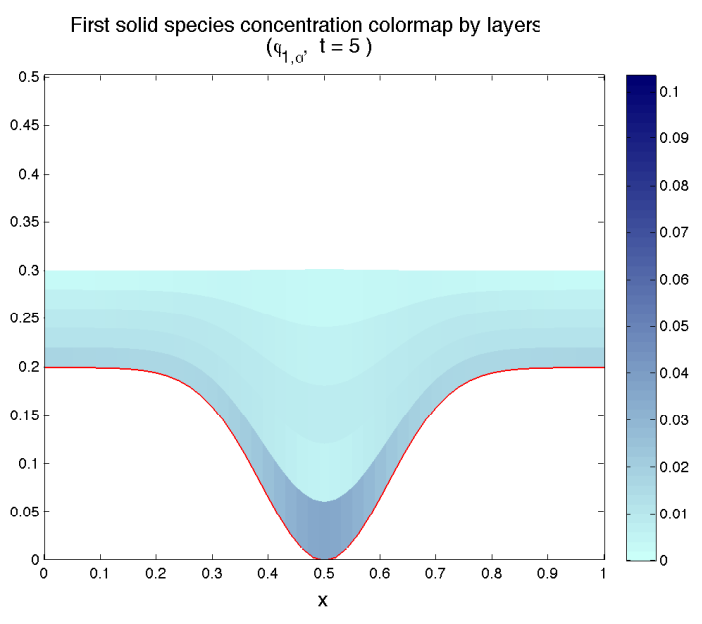

(b) $\phi_{1, \alpha}(t=5 \mathrm{~s})$.

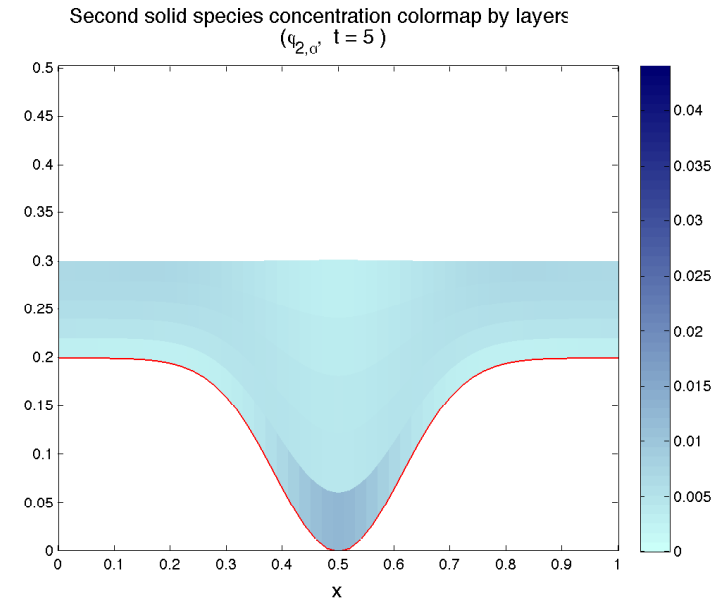

(d) $\phi_{2, \alpha}(t=5 \mathrm{~s})$.

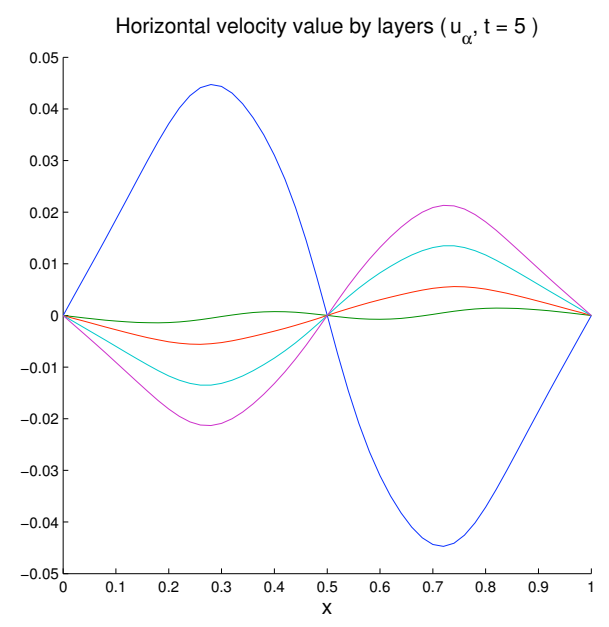

(f) $u_{\alpha}(t=5 \mathrm{~s})$.

Figure 10: Test 2: solid species concentrations and horizontal velocities by layers at the time $t=5$ $\mathrm{s}$, with closed end walls. 


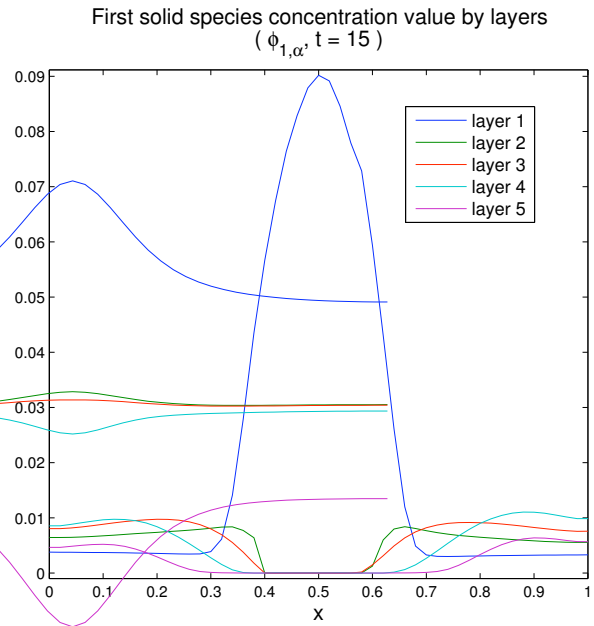

(a) $\phi_{1, \alpha}(t=15 \mathrm{~s})$.

Second solid species concentration value by layers $\left(\phi_{2, \alpha}, t=15\right)$

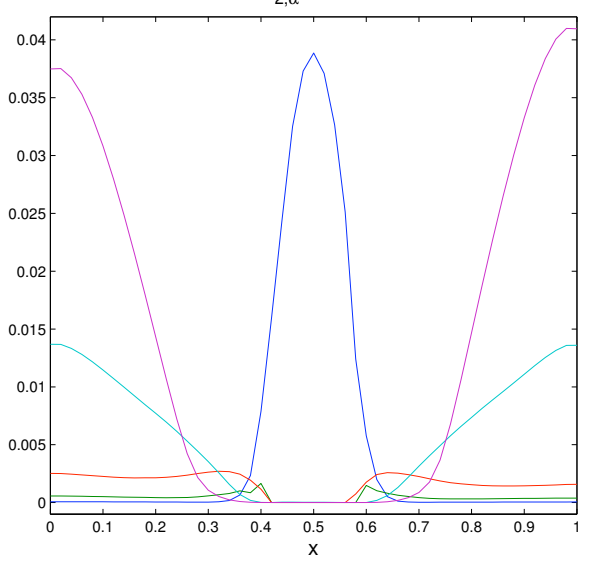

(c) $\phi_{2, \alpha}(t=15 \mathrm{~s})$.

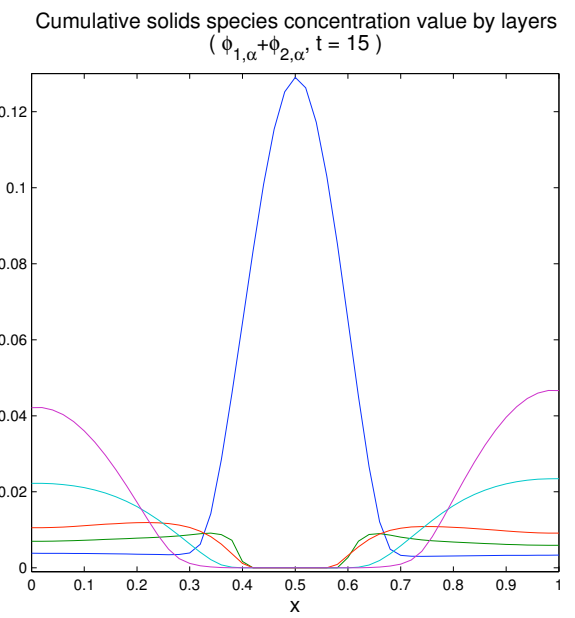

(e) $\left(\phi_{1, \alpha}+\phi_{2, \alpha}\right)(t=15 \mathrm{~s})$.

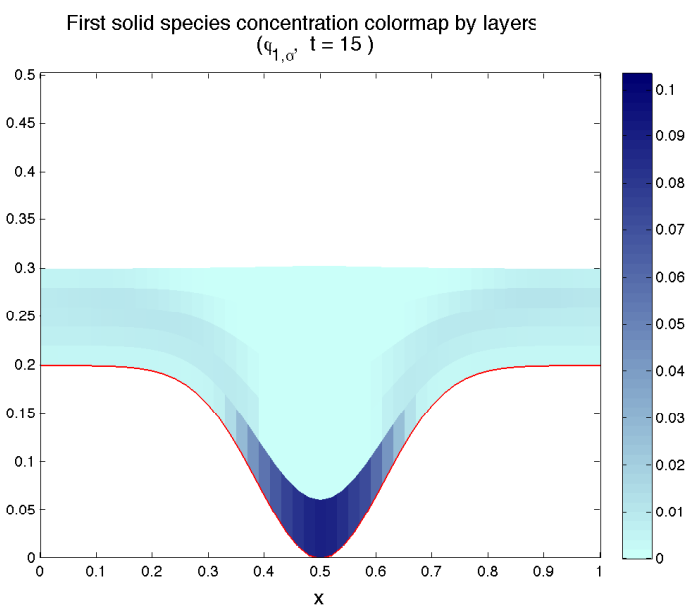

(b) $\phi_{1, \alpha}(t=15 \mathrm{~s})$.

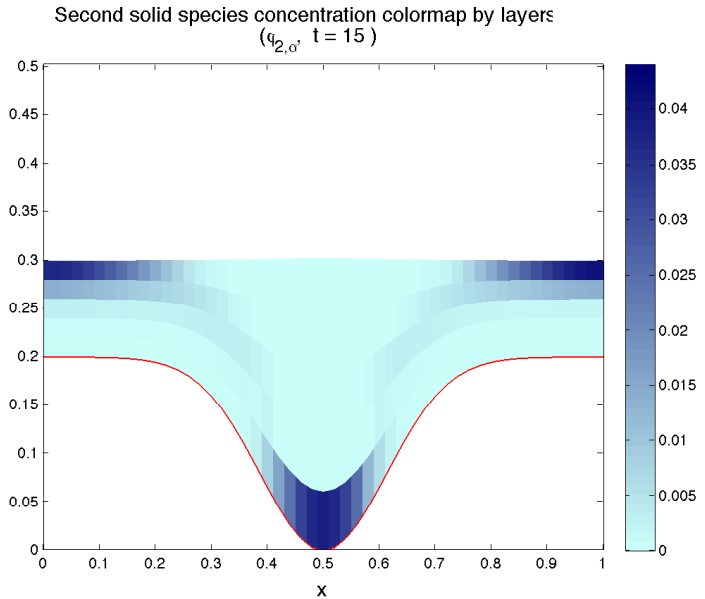

(d) $\phi_{2, \alpha}(t=15 \mathrm{~s})$.

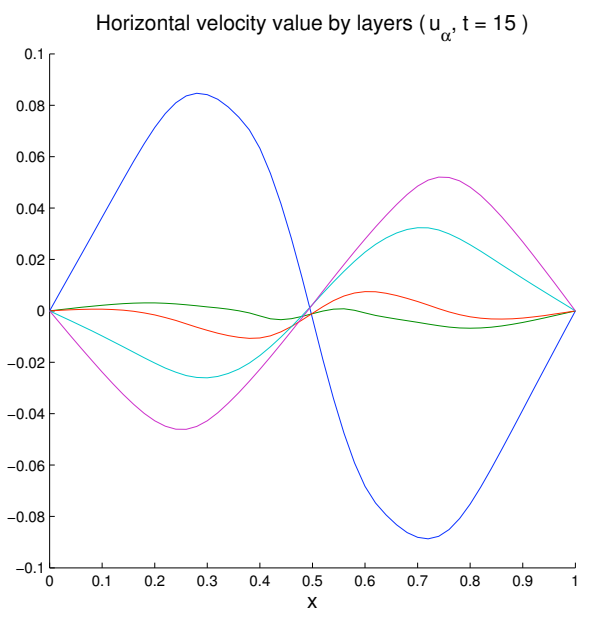

(f) $u_{\alpha}(t=15 \mathrm{~s})$.

Figure 11: Test 2: solid species concentrations and horizontal velocities by layers at the time $t=15$ $\mathrm{s}$, with closed end walls. 


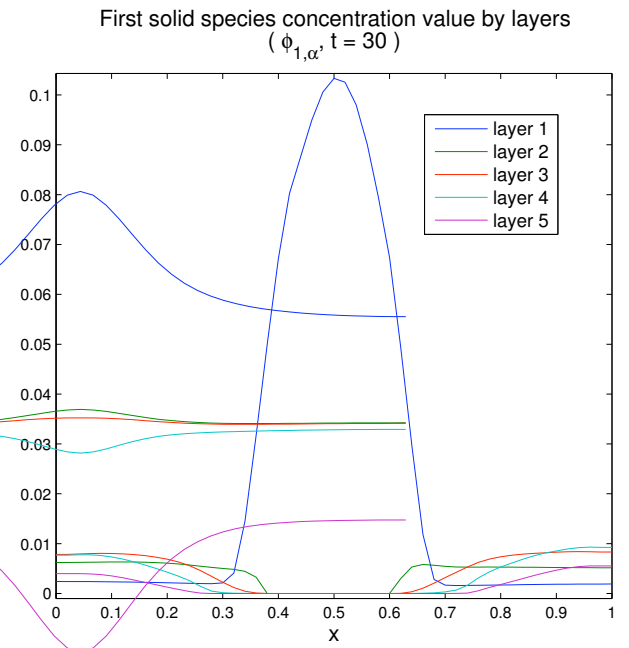

(a) $\phi_{1, \alpha}(t=30 \mathrm{~s})$.

Second solid species concentration value by layers $\left(\phi_{2, \alpha}, t=30\right)$

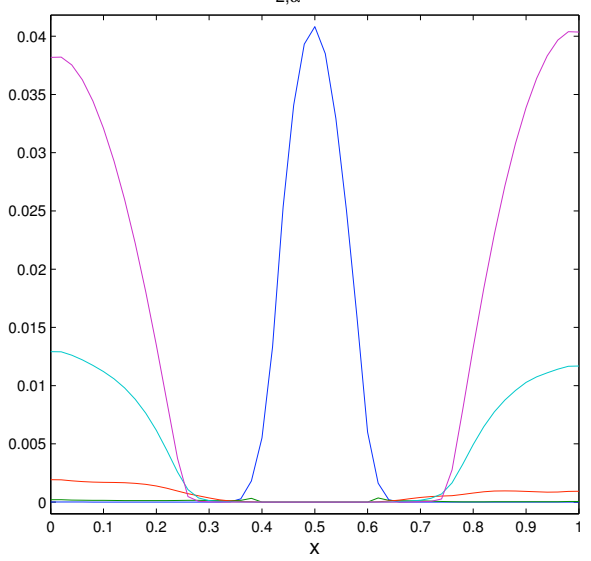

(c) $\phi_{2, \alpha}(t=30 \mathrm{~s})$.

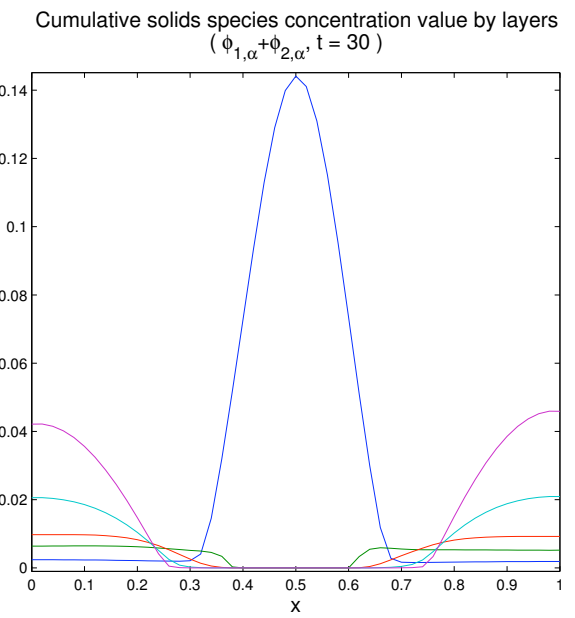

(e) $\left(\phi_{1, \alpha}+\phi_{2, \alpha}\right)(t=30 \mathrm{~s})$.

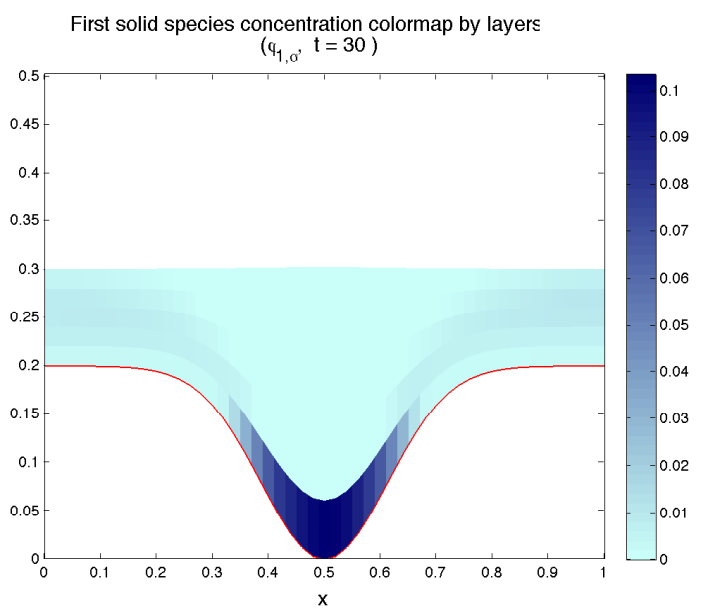

(b) $\phi_{1, \alpha}(t=30 \mathrm{~s})$.

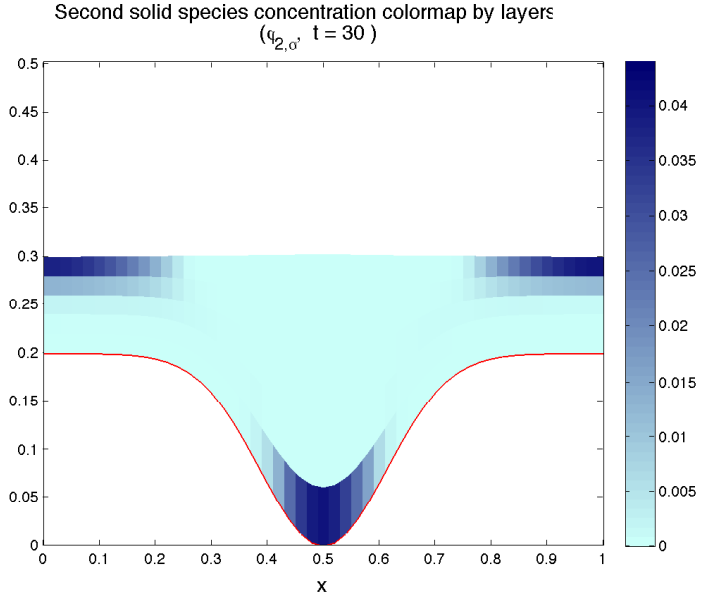

(d) $\phi_{2, \alpha}(t=30 \mathrm{~s})$.

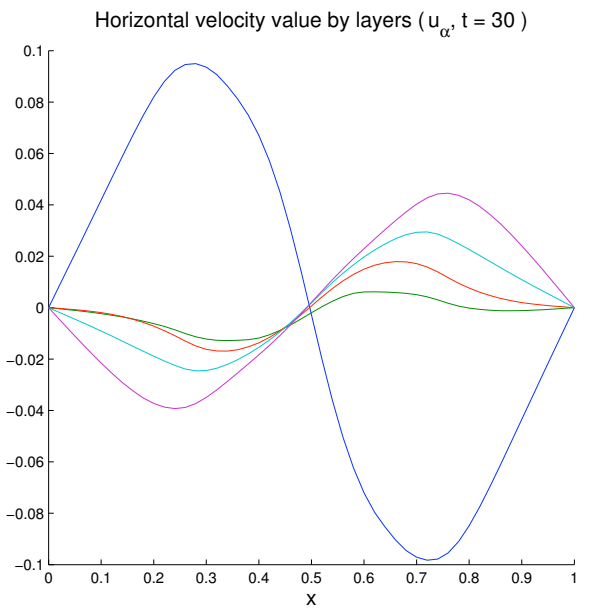

(f) $u_{\alpha}(t=30 \mathrm{~s})$.

Figure 12: Test 2: solid species concentrations and horizontal velocities by layers at the time $t=30$ $\mathrm{s}$, with closed end walls. 


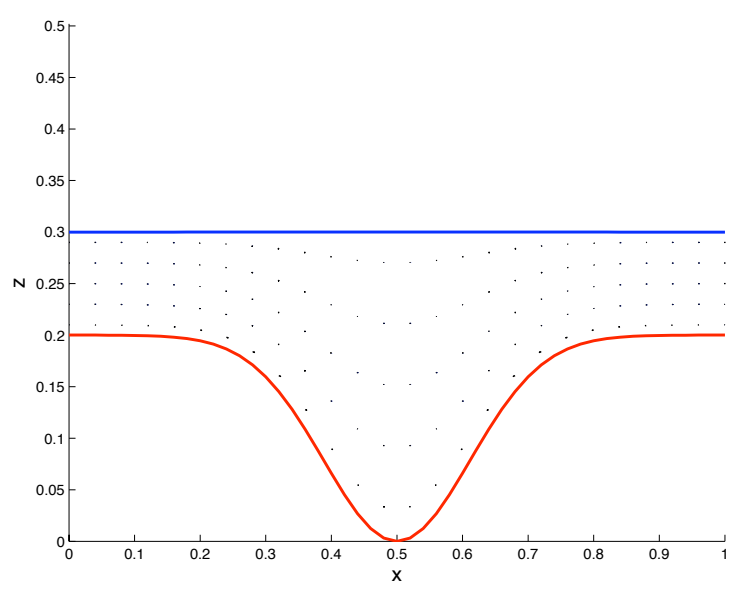

(a) $t=1 \mathrm{~s}$.

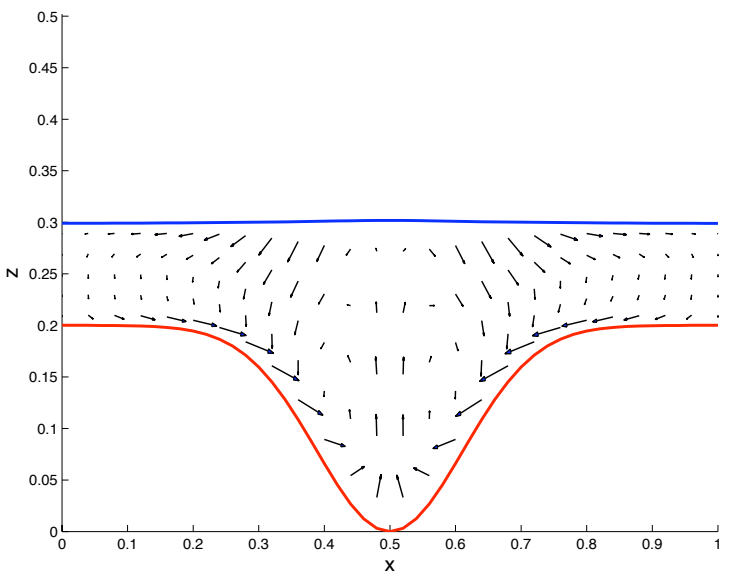

(c) $t=15 \mathrm{~s}$.

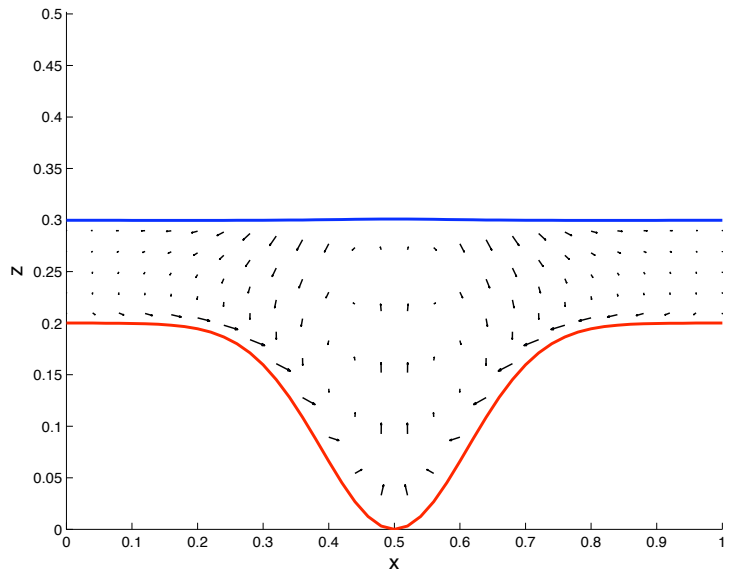

(b) $t=5 \mathrm{~s}$.

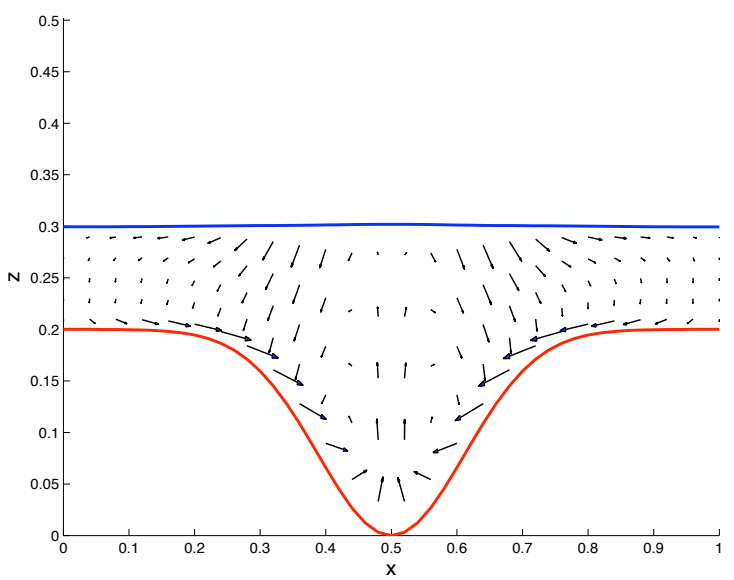

(d) $t=30 \mathrm{~s}$.

Figure 13: Test 2: velocity vectors $\left(u_{\alpha}, \varphi_{\alpha}\right)^{\mathrm{T}}$ by layers at the times $t=1,5,15,30 \mathrm{~s}$, with closed end walls. (Vector length divided by 2 ) 


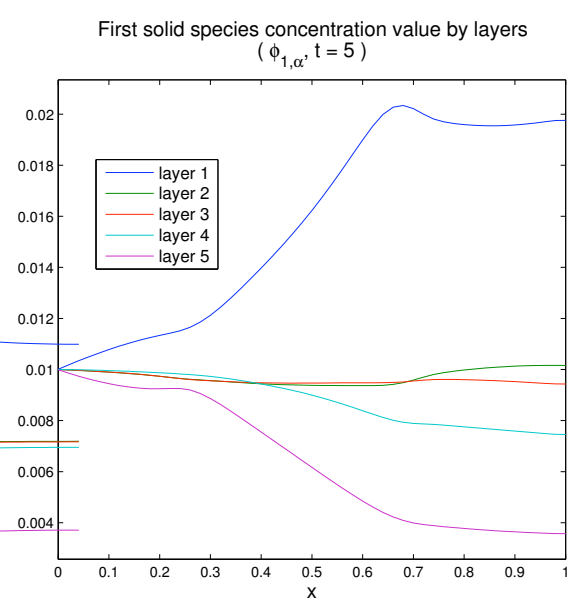

(a) $\phi_{1, \alpha}(t=5 \mathrm{~s})$.

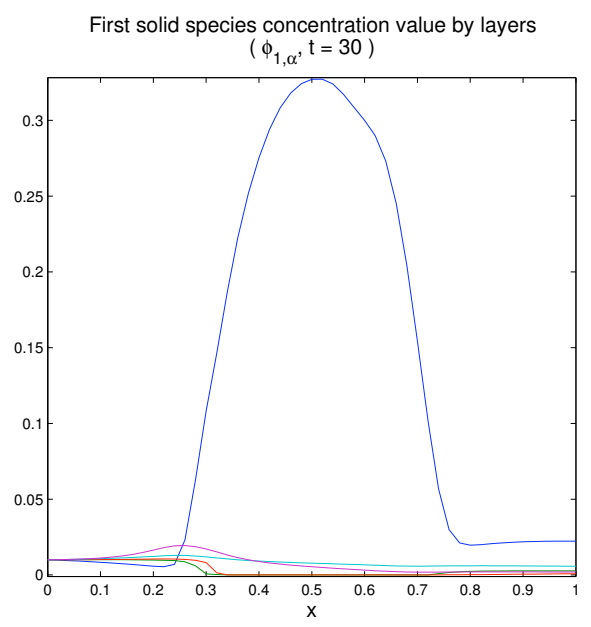

(c) $\phi_{1, \alpha}(t=30 \mathrm{~s})$.

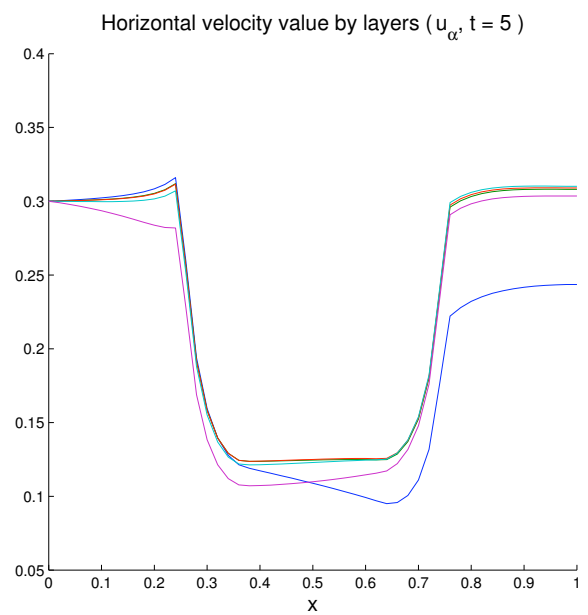

(e) $u_{\alpha}(t=5 \mathrm{~s})$.

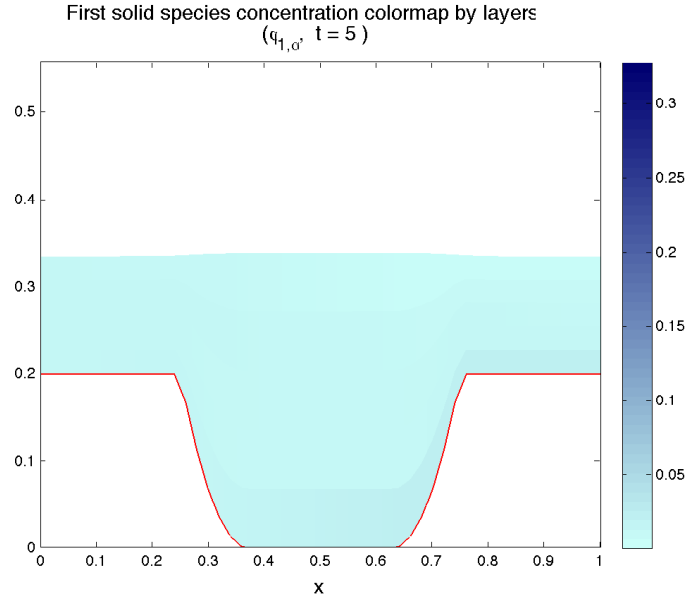

(b) $\phi_{1, \alpha}(t=5 \mathrm{~s})$.

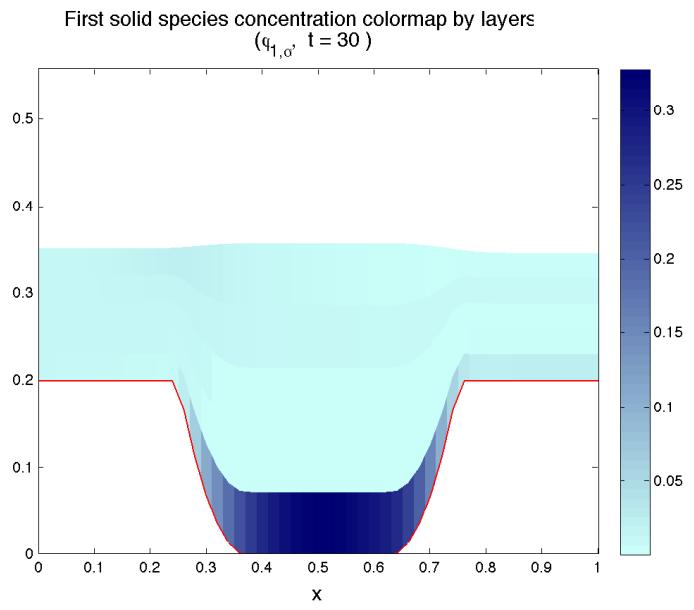

(d) $\phi_{1, \alpha}(t=30 \mathrm{~s})$.

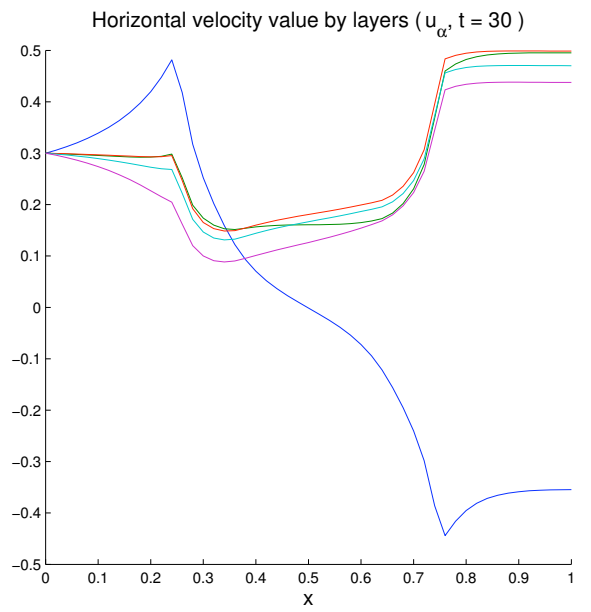

(f) $u_{\alpha}(t=30 \mathrm{~s})$

Figure 14: Test 3: first solid species concentrations and horizontal velocities by layers at the times $t=5,30 \mathrm{~s}$, for open end walls with $\phi_{1, b, \alpha}=0.01, \phi_{2, b, \alpha}=0.005$ and $u_{b, \alpha}=0.3 \mathrm{~ms}^{-1}$. 


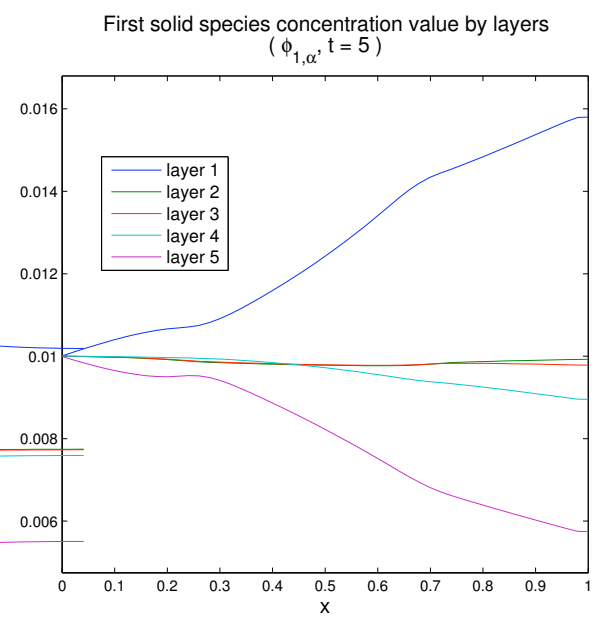

(a) $\phi_{1, \alpha}(t=5 \mathrm{~s})$.

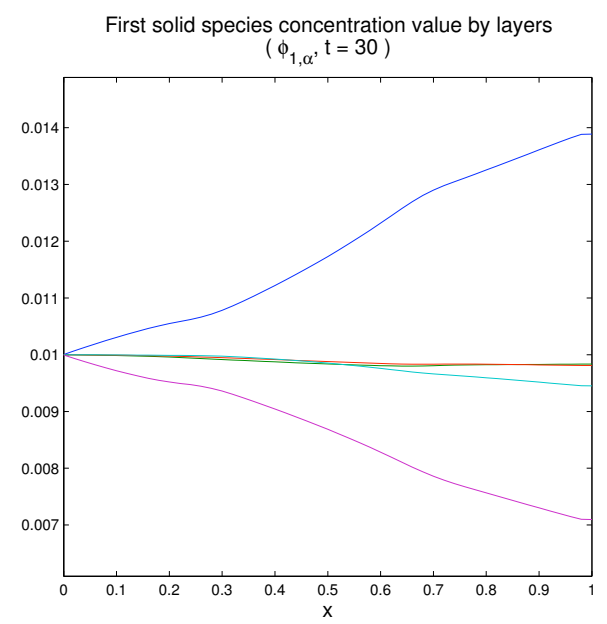

(c) $\phi_{1, \alpha}(t=30 \mathrm{~s})$.

Horizontal velocity value by layers $\left(\mathrm{u}_{\alpha}, \mathrm{t}=5\right)$

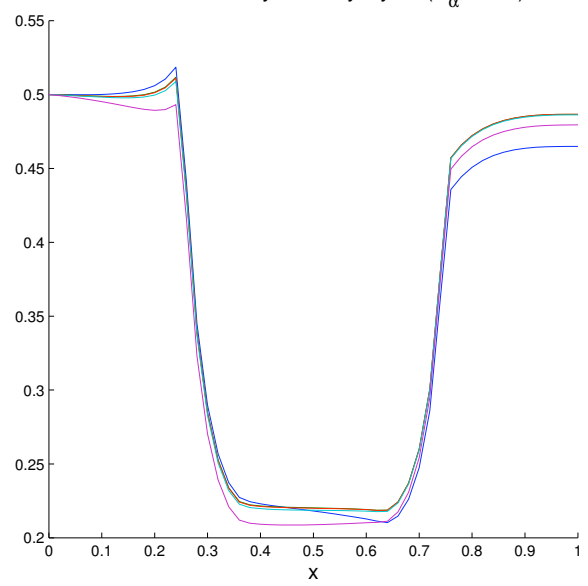

(e) $u_{\alpha}(t=5 \mathrm{~s})$.

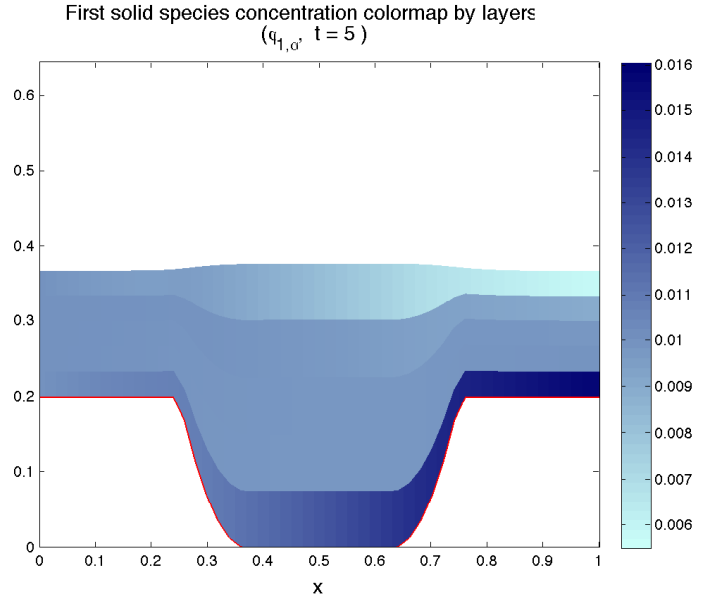

(b) $\phi_{1, \alpha}(t=5 \mathrm{~s})$.

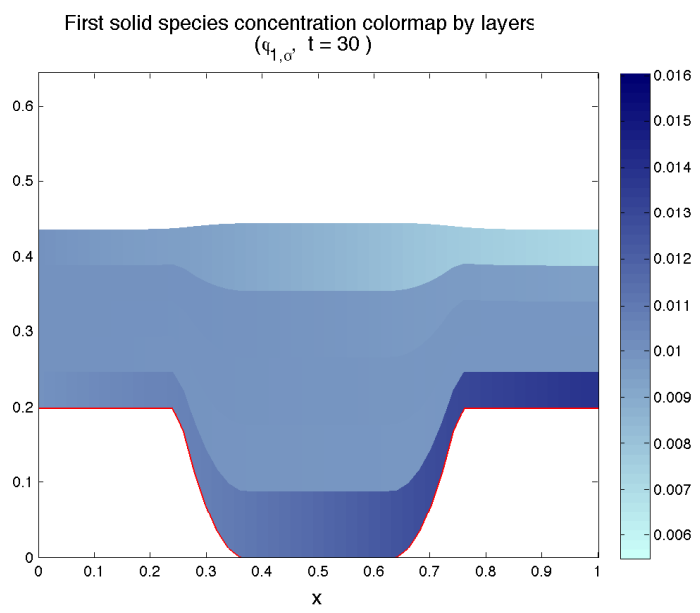

(d) $\phi_{1, \alpha}(t=30 \mathrm{~s})$.

Horizontal velocity value by layers $\left(\mathrm{u}_{\alpha}, \mathrm{t}=30\right)$

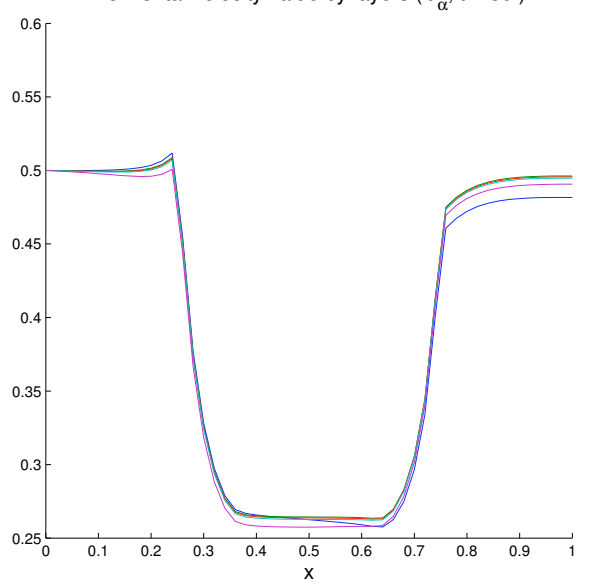

(f) $u_{\alpha}(t=30 \mathrm{~s})$.

Figure 15: Test 3: first solid species concentrations and horizontal velocities by layers at the times $t=5,30 \mathrm{~s}$, for open end walls with $\phi_{1, b, \alpha}=0.01, \phi_{2, b, \alpha}=0.005$ and $u_{b, \alpha}=0.5 \mathrm{~ms}^{-1}$. 


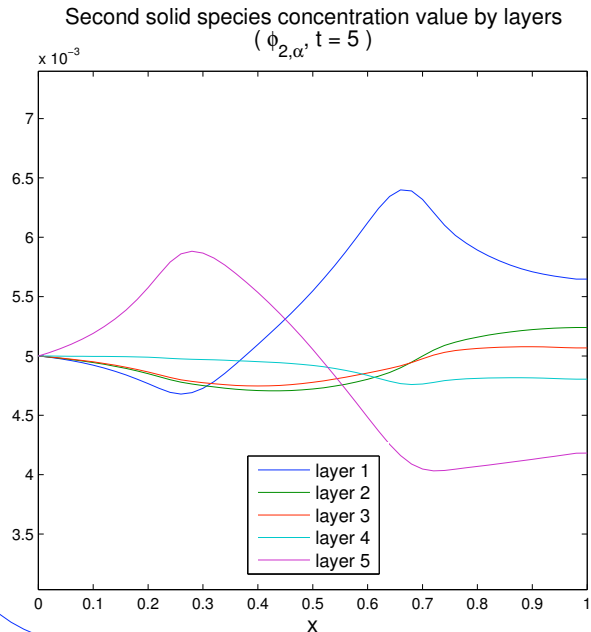

(a) $\phi_{2, \alpha}(t=5 \mathrm{~s})$.

Second solid species concentration value by layers

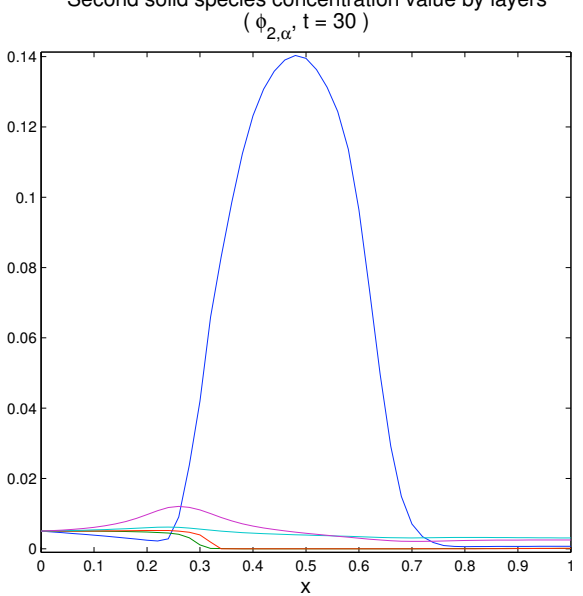

(c) $\phi_{2, \alpha}(t=30 \mathrm{~s})$.

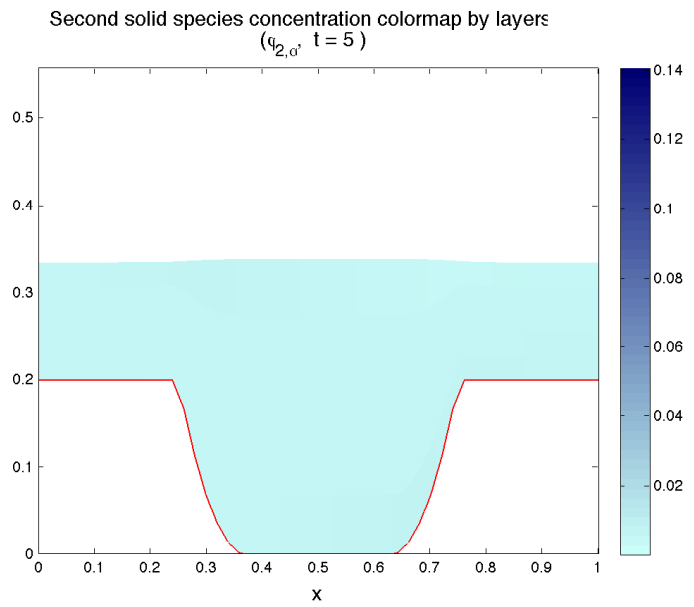

(b) $\phi_{2, \alpha}(t=5 \mathrm{~s})$.

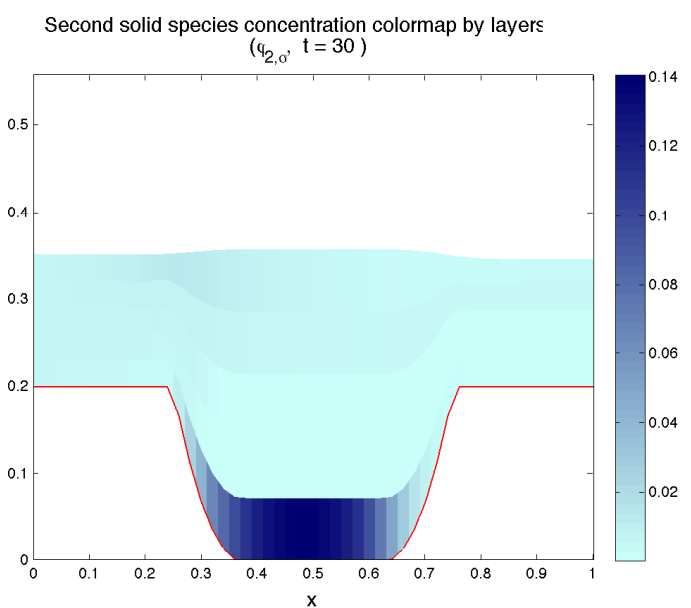

(d) $\phi_{2, \alpha}(t=30 \mathrm{~s})$.

Figure 16: Test 3: second solid species concentrations by layers at the times $t=5,30 \mathrm{~s}$, for open end walls with $\phi_{1, b, \alpha}=0.01, \phi_{2, b, \alpha}=0.005$ and $u_{b, \alpha}=0.3 \mathrm{~ms}^{-1}$. 


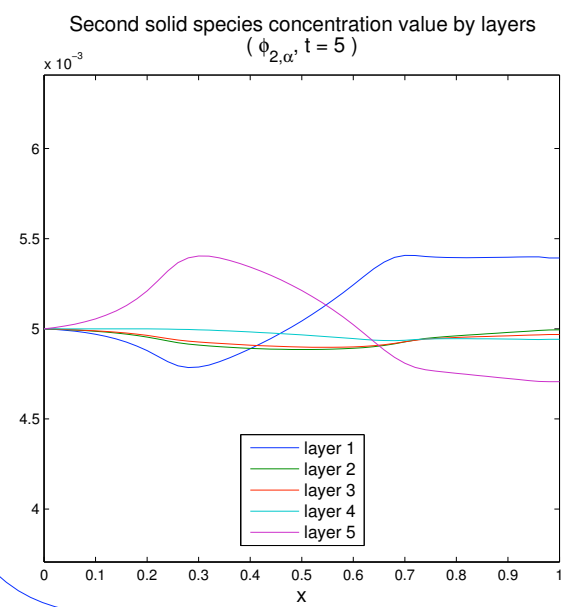

(a) $\phi_{2, \alpha}(t=5 \mathrm{~s})$.

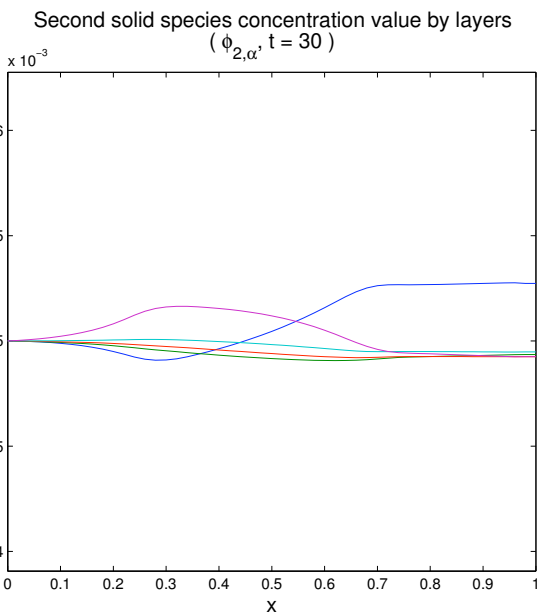

(c) $\phi_{2, \alpha}(t=30 \mathrm{~s})$.

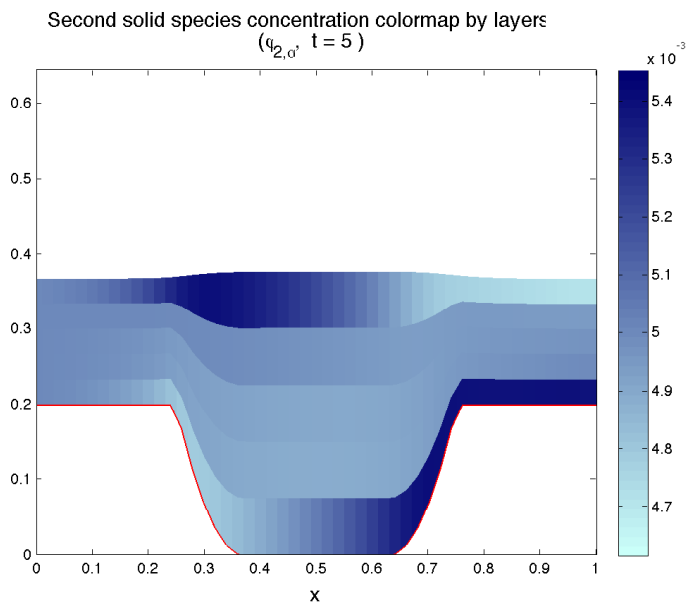

(b) $\phi_{2, \alpha}(t=5 \mathrm{~s})$.

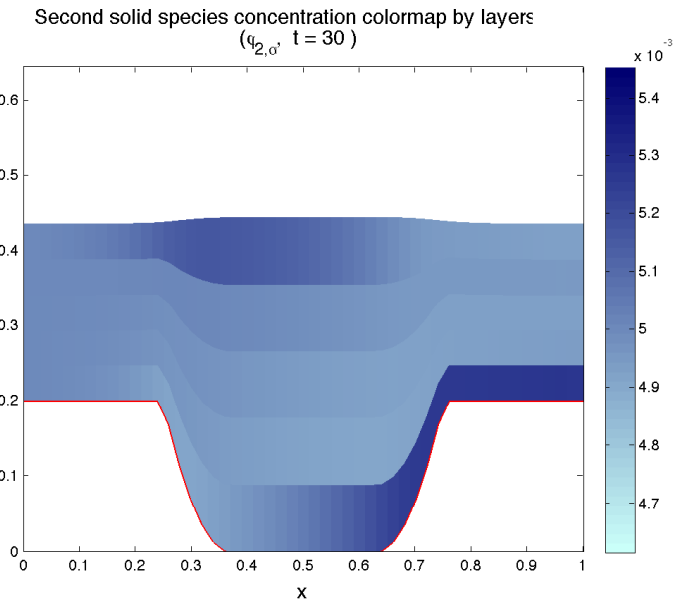

(d) $\phi_{2, \alpha}(t=30 \mathrm{~s})$.

Figure 17: Test 3: second solid species concentrations by layers at the times $t=5,30 \mathrm{~s}$, for open end walls with $\phi_{1, b, \alpha}=0.01, \phi_{2, b, \alpha}=0.005$ and $u_{b, \alpha}=0.5 \mathrm{~ms}^{-1}$. 


\section{Conclusions}

In this work we propose a multilayer approach of the MLB model to study polydisperse sedimentation processes in free-surface shallow domains. Then, a hydrostatic pressure is considered. This technique allows us to keep information on the vertical distribution of solid particle in the mixture. This is crucial in order to take into account the segregation of the particle species from each other, and from the fluid, taking place in the vertical direction of the gravity body force only. We have shown that the proposed model can be written under the structure of a system of nonlinear first-order balance equations with nonconservative products (equation (3.10)) with $(N+1) M+1$ unknowns, where $N$ is the number of species and $M$ the number of layers. Nevertheless, to approximate it, we do not consider a numerical solver applied directly to this system. We propose a different technique that allows us to diminish the number of unknowns of the effective transport matrix of the system. Concretely, by taking into account that the model implies system (4.2) with $2 M+1$ unknowns, we combine an approximation of this reduced system with an upwind approximation of the horizontal flux corresponding to the continuity equation for each one of the species. In the second step of the numerical solver, we include the effect of the vertical fluxes corresponding to the MLB model. Numerical results suggest that the multilayer shallow water model could be adequate in situations where the settling takes place from a suspension that undergoes horizontal movement. Typical situations of that kind should include the transport and deposition

of suspended particulate matter in rivers and estuaries. To this end, the model should be extended to two horizontal space dimensions, and additional mechanisms of sediment erosion should be built in. Experimental and theoretical analyses of such scenarios are cited in Section 1.1.

\section{A Appendix: definition in terms of the variable $w$}

Considering the unknown $\boldsymbol{w}$ as defined in (3.15), the equation (3.9) is merely rewritten as

$$
\partial_{t} h+\partial_{x}\left(\sum_{\beta=1}^{M} l_{\beta} \frac{q_{\beta}}{\rho_{\beta}(\Phi)}\right)=0 .
$$

Next, to handle the equation (3.3), we here expand the right-hand side for $j=1, \ldots, N$ :

$$
\begin{gathered}
\Delta_{-}^{\alpha} H_{j, \alpha+1 / 2}(\Phi)=\sum_{\gamma=1}^{M}\left(-\theta_{j, \alpha-1 / 2} \xi_{\alpha-1, \gamma} \phi_{j, \alpha-1}+\left(\theta_{j, \alpha+1 / 2} \xi_{\alpha, \gamma}-\left(1-\theta_{j, \alpha-1 / 2}\right) \xi_{\alpha-1, \gamma}\right) \phi_{j, \alpha}+\right. \\
\left.\left(1-\theta_{j, \alpha+1 / 2}\right) \xi_{\alpha, \gamma} \phi_{j, \alpha+1}\right) \partial_{x}\left(h u_{\gamma}\right)-\Delta_{-}^{\alpha} f_{j, \alpha+1 / 2}(\Phi) .
\end{gathered}
$$

Using that equality, we can rewrite equation (3.3) as

$$
\begin{aligned}
& \partial_{t} r_{j, \alpha}+\partial_{x}\left(r_{j, \alpha} u_{\alpha}\right)-\frac{1}{h l_{\alpha}} \sum_{\gamma=1}^{M}\left(-\theta_{j, \alpha-1 / 2} \xi_{\alpha-1, \gamma} r_{j, \alpha-1}+\left(\theta_{j, \alpha+1 / 2} \xi_{\alpha, \gamma}-\left(1-\theta_{j, \alpha-1 / 2}\right) \xi_{\alpha-1, \gamma}\right) r_{j, \alpha}\right. \\
& \left.+\left(1-\theta_{j, \alpha+1 / 2}\right) \xi_{\alpha, \gamma} r_{j, \alpha+1}\right) \partial_{x}\left(h u_{\gamma}\right)=-\frac{1}{l_{\alpha}} \Delta_{-}^{\alpha} f_{j, \alpha+1 / 2}(\Phi) .
\end{aligned}
$$

From now on, we will omit here the argument " $\Phi$ " in $\rho_{\alpha}(\Phi)$. Then, concerning the case of equation (3.6), which we can rewrite as

$$
\partial_{t}\left(h \rho_{\alpha} u_{\alpha}\right)+\partial_{x}\left(h \rho_{\alpha} u_{\alpha}^{2}\right)+\partial_{x}\left(h p_{\mathrm{S}}+g h^{2}\left[\frac{1}{2} l_{\alpha} \rho_{\alpha}+\sum_{\beta=\alpha+1}^{M} l_{\beta} \rho_{\beta}\right]\right)
$$




$$
=\sum_{j=0}^{N} \frac{\rho_{j}}{l_{\alpha}} \Delta_{-}^{\alpha}\left(u_{\alpha+1 / 2} H_{j, \alpha+1 / 2}(\Phi)\right)+\left(p_{\mathrm{S}}+g h \sum_{\beta=\alpha+1}^{M} l_{\beta} \rho_{\beta}\right) \partial_{x} h-g h L_{\alpha-1} \rho_{\alpha} \partial_{x} h-g h \rho_{\alpha} \partial_{x} z_{\mathrm{B}},
$$

let us notice that

$$
\Delta_{-}^{\alpha}\left(u_{\alpha+1 / 2} H_{0, \alpha+1 / 2}(\Phi)\right)=-\sum_{j=1}^{N} \Delta_{-}^{\alpha}\left(u_{\alpha+1 / 2} H_{j, \alpha+1 / 2}(\Phi)\right)+\Delta_{-}^{\alpha}\left(u_{\alpha+1 / 2} G_{\alpha+1 / 2}\right) .
$$

Then we get

$$
\sum_{j=0}^{N} \frac{\rho_{j}}{l_{\alpha}} \Delta_{-}^{\alpha}\left(u_{\alpha+1 / 2} H_{j, \alpha+1 / 2}(\Phi)\right)=\sum_{j=1}^{N} \frac{\bar{\rho}_{j}}{l_{\alpha}} \Delta_{-}^{\alpha}\left(u_{\alpha+1 / 2} H_{j, \alpha+1 / 2}(\Phi)\right)+\frac{\rho_{0}}{l_{\alpha}} \Delta_{-}^{\alpha}\left(u_{\alpha+1 / 2} G_{\alpha+1 / 2}\right),
$$

where we recall that $\bar{\rho}_{j}=\rho_{j}-\rho_{0}$ for all $j=1, \ldots, N$. In addition, expanding the quantity

$$
\Delta_{-}^{\alpha}\left(u_{\alpha+1 / 2} G_{\alpha+1 / 2}\right)=\sum_{\gamma=1}^{M} \Delta_{-}^{\alpha}\left(\left(\varepsilon_{\alpha+1 / 2} u_{\alpha}+\left(1-\varepsilon_{\alpha+1 / 2}\right) u_{\alpha+1}\right) \xi_{\alpha, \gamma}\right) \partial_{x}\left(h u_{\gamma}\right)
$$

as well as the following one,

$$
\begin{aligned}
& \Delta_{-}^{\alpha}\left(u_{\alpha+1 / 2} H_{j, \alpha+1 / 2}(\Phi)\right) \\
& =\sum_{\gamma=1}^{M} \Delta_{-}^{\alpha}\left(\left(\varepsilon_{\alpha+1 / 2} u_{\alpha}+\left(1-\varepsilon_{\alpha+1 / 2}\right) u_{\alpha+1}\right)\left(\theta_{j, \alpha+1 / 2} \phi_{j, \alpha}+\left(1-\theta_{j, \alpha+1 / 2}\right) \phi_{j, \alpha+1}\right) \xi_{\alpha, \gamma}\right) \partial_{x}\left(h u_{\gamma}\right) \\
& \quad-\Delta_{-}^{\alpha}\left(\left(\varepsilon_{\alpha+1 / 2} u_{\alpha}+\left(1-\varepsilon_{\alpha+1 / 2}\right) u_{\alpha+1}\right) f_{j, \alpha+1 / 2}(\Phi)\right),
\end{aligned}
$$

we may rewrite equation (3.6) as follows:

$$
\begin{aligned}
& \partial_{t}\left(h \rho_{\alpha} u_{\alpha}\right)+\partial_{x}\left(h \rho_{\alpha} u_{\alpha}^{2}+g h^{2}\left[\frac{1}{2} l_{\alpha} \rho_{\alpha}+\sum_{\beta=\alpha+1}^{M} l_{\beta} \rho_{\beta}\right]\right)+g h\left(L_{\alpha-1} \rho_{\alpha}-\sum_{\beta=\alpha+1}^{M} l_{\beta} \rho_{\beta}\right) \partial_{x} h \\
& -\sum_{\gamma=1}^{M} \frac{1}{h^{2} l_{\alpha}} \boldsymbol{b}_{\alpha, \gamma}^{(1)} \partial_{x}\left(h u_{\gamma}\right)=\frac{1}{h^{2} l_{\alpha}} \boldsymbol{c}_{\alpha}^{(1)}-g h \rho_{\alpha} \partial_{x} z_{\mathrm{B}}-h \partial_{x} p_{\mathrm{S}} .
\end{aligned}
$$

More precisely, recalling that

$$
\rho_{\gamma}(\Phi)=\rho_{0}+\frac{1}{h} \sum_{j=1}^{N} \bar{\rho}_{j} r_{j, \gamma} \quad \text { for all } \gamma=1, \ldots, M
$$

so that

$$
\partial_{x}\left(h u_{\gamma}\right)=\partial_{x}\left(\frac{q_{\gamma}}{\rho_{\gamma}}\right)=\frac{1}{\rho_{\gamma}} \partial_{x} q_{\gamma}-\frac{q_{\gamma}}{\rho_{\gamma}^{2}} \partial_{x} \rho_{\gamma}=\frac{1}{\rho_{\gamma}} \partial_{x} q_{\gamma}+\frac{\left(\rho_{\gamma}-\rho_{0}\right) q_{\gamma}}{h \rho_{\gamma}^{2}} \partial_{x} h-\frac{q_{\gamma}}{h \rho_{\gamma}^{2}} \sum_{j=1}^{N} \bar{\rho}_{j} \partial_{x} r_{j, \gamma}
$$

we can finally rewrite equation (3.6) as

$$
\begin{aligned}
& \partial_{t} q_{\alpha}+\partial_{x}\left(\frac{q_{\alpha}^{2}}{h \rho_{\alpha}}+g h^{2}\left[\frac{1}{2} l_{\alpha} \rho_{\alpha}+\sum_{\beta=\alpha+1}^{M} l_{\beta} \rho_{\beta}\right]\right)+g h\left(L_{\alpha-1} \rho_{\alpha}-\sum_{\beta=\alpha+1}^{M} l_{\beta} \rho_{\beta}\right) \partial_{x} h \\
& -\sum_{\gamma=1}^{M} \frac{1}{h^{2} l_{\alpha}} \boldsymbol{b}_{\alpha, \gamma}^{(1)}\left(\frac{1}{\rho_{\gamma}} \partial_{x} q_{\gamma}+\frac{\left(\rho_{\gamma}-\rho_{0}\right) q_{\gamma}}{h \rho_{\gamma}^{2}} \partial_{x} h-\frac{q_{\gamma}}{h \rho_{\gamma}^{2}} \sum_{j=1}^{N} \bar{\rho}_{j} \partial_{x} r_{j, \gamma}\right) \\
& =\frac{1}{h^{2} l_{\alpha}} \boldsymbol{c}_{\alpha}^{(1)}-g h \rho_{\alpha} \partial_{x} z_{\mathrm{B}}-h \partial_{x} p_{\mathrm{S}},
\end{aligned}
$$


where we define

$$
\begin{aligned}
\boldsymbol{b}_{\alpha, \gamma}^{(1)} & :=\Delta_{-}^{\alpha}\left(\left(\frac{\varepsilon_{\alpha+1 / 2} q_{\alpha}}{\rho_{\alpha}}+\frac{\left(1-\varepsilon_{\alpha+1 / 2}\right) q_{\alpha+1}}{\rho_{\alpha+1}}\right) \xi_{\alpha, \gamma}\left[h \rho_{\alpha+1}+\sum_{j=1}^{N} \bar{\rho}_{j} \theta_{j, \alpha+1 / 2}\left(r_{j, \alpha}-r_{j, \alpha+1}\right)\right]\right), \\
\boldsymbol{c}_{\alpha}^{(1)} & :=-\Delta_{-}^{\alpha}\left(\left(\frac{\varepsilon_{\alpha+1 / 2} q_{\alpha}}{\rho_{\alpha}(\Phi)}+\frac{\left(1-\varepsilon_{\alpha+1 / 2}\right) q_{\alpha+1}}{\rho_{\alpha+1}(\Phi)}\right) h \sum_{j=0}^{N} \rho_{j} f_{j, \alpha+1 / 2}(\Phi)\right) .
\end{aligned}
$$

To complete the system, we rewrite equation (A.2) as

$$
\partial_{t} r_{j, \alpha}+\partial_{x}\left(\frac{q_{\alpha} r_{j, \alpha}}{h \rho_{\alpha}}\right)-\sum_{\gamma=1}^{M} \frac{1}{h l_{\alpha}} \boldsymbol{b}_{j, \alpha, \gamma}^{(2)}\left(\frac{1}{\rho_{\gamma}} \partial_{x} q_{\gamma}+\frac{\left(\rho_{\gamma}-\rho_{0}\right) q_{\gamma}}{h \rho_{\gamma}^{2}} \partial_{x} h-\frac{q_{\gamma}}{h \rho_{\gamma}^{2}} \sum_{k=1}^{N} \bar{\rho}_{k} \partial_{x} r_{k, \gamma}\right)=\frac{1}{h l_{\alpha}} \boldsymbol{c}_{j, \alpha}^{(2)},
$$

where we define

$$
\begin{aligned}
\boldsymbol{b}_{j, \alpha, \gamma}^{(2)}:= & -\theta_{j, \alpha-1 / 2} \xi_{\alpha-1, \gamma} r_{j, \alpha-1}+\left(\theta_{j, \alpha+1 / 2} \xi_{\alpha, \gamma}-\left(1-\theta_{j, \alpha-1 / 2}\right) \xi_{\alpha-1, \gamma}\right) r_{j, \alpha} \\
& +\left(1-\theta_{j, \alpha+1 / 2}\right) \xi_{\alpha, \gamma} r_{j, \alpha+1}, \\
\boldsymbol{c}_{j, \alpha}^{(2)}:= & -h \Delta_{-}^{\alpha} f_{j, \alpha+1 / 2}(\Phi) .
\end{aligned}
$$

The system of $(N+1) M+1$ equations made up of the equations (A.1), (A.3) and (A.4) has the form (3.14) with the notation (3.15), (3.16) for the unknown vector. The scalar function $H$ is actually nothing else but the bottom function (i.e., $H=z_{\mathrm{B}}$ ). Let us introduce the integer index

$$
\mathrm{I}(\alpha, j)=(\alpha-1) N+j+M+1 \text { for } \alpha=1, \ldots, M \text { and } j=1, \ldots, N,
$$

which defines a one-to-one function I $:\{1, \ldots, M\} \times\{1, \ldots, N\} \rightarrow\{M+2, \ldots,(N+1) M+1\}$. Note that I has a well-defined inverse, which we denote by I. We now define the vectors $\boldsymbol{S}(\boldsymbol{w})=$ $\left(S_{\nu}(\boldsymbol{w})\right)_{\nu=1, \ldots,(N+1) M+1}, \boldsymbol{G}(\boldsymbol{w})=\left(G_{\nu}(\boldsymbol{w})\right)_{\nu=1, \ldots,(N+1) M+1}$ and $\boldsymbol{F}(\boldsymbol{w})=\left(F_{\nu}(\boldsymbol{w})\right)_{\nu=1, \ldots,(N+1) M+1}$.

$$
\begin{aligned}
& S_{\nu}(\boldsymbol{w})= \begin{cases}0 & \text { for } \nu=1 \text { and } \nu=M+2, \ldots,(N+1) M+1, \\
-g h \rho_{\alpha}, \quad \alpha=\nu-1 & \text { for } \nu=2, \cdots, M+1,\end{cases} \\
& G_{\nu}(\boldsymbol{w})= \begin{cases}G_{M+1 / 2}-G_{1 / 2} & \text { for } \nu=1, \\
\frac{\boldsymbol{c}_{\alpha}^{(1)}}{h^{2} l_{\alpha}}-h \partial_{x} p_{\mathrm{S}}, \quad \alpha=\nu-1 & \text { for } \nu=2, \ldots, M+1, \\
\frac{\boldsymbol{c}_{j, \alpha}^{(2)}}{h l_{\alpha}}, \quad(\alpha, j)=\hat{\mathrm{I}}(\nu) & \text { for } \nu=M+2, \ldots,(N+1) M+1,\end{cases}
\end{aligned}
$$

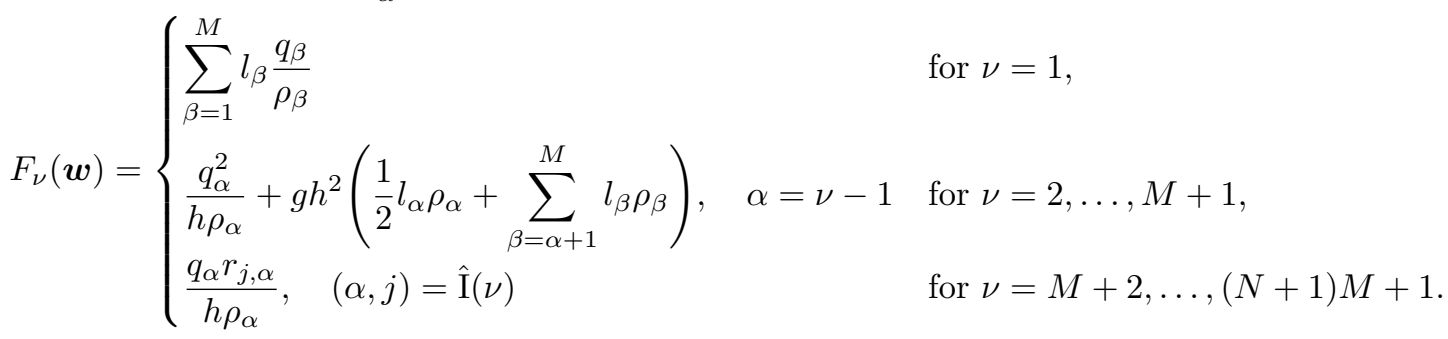


The matrix $\boldsymbol{B}(\boldsymbol{w})=\left(B_{\nu, \mu}(\boldsymbol{w})\right)_{\nu, \mu=1, \ldots,(N+1) M+1}$ within the equation (3.14) is defined by

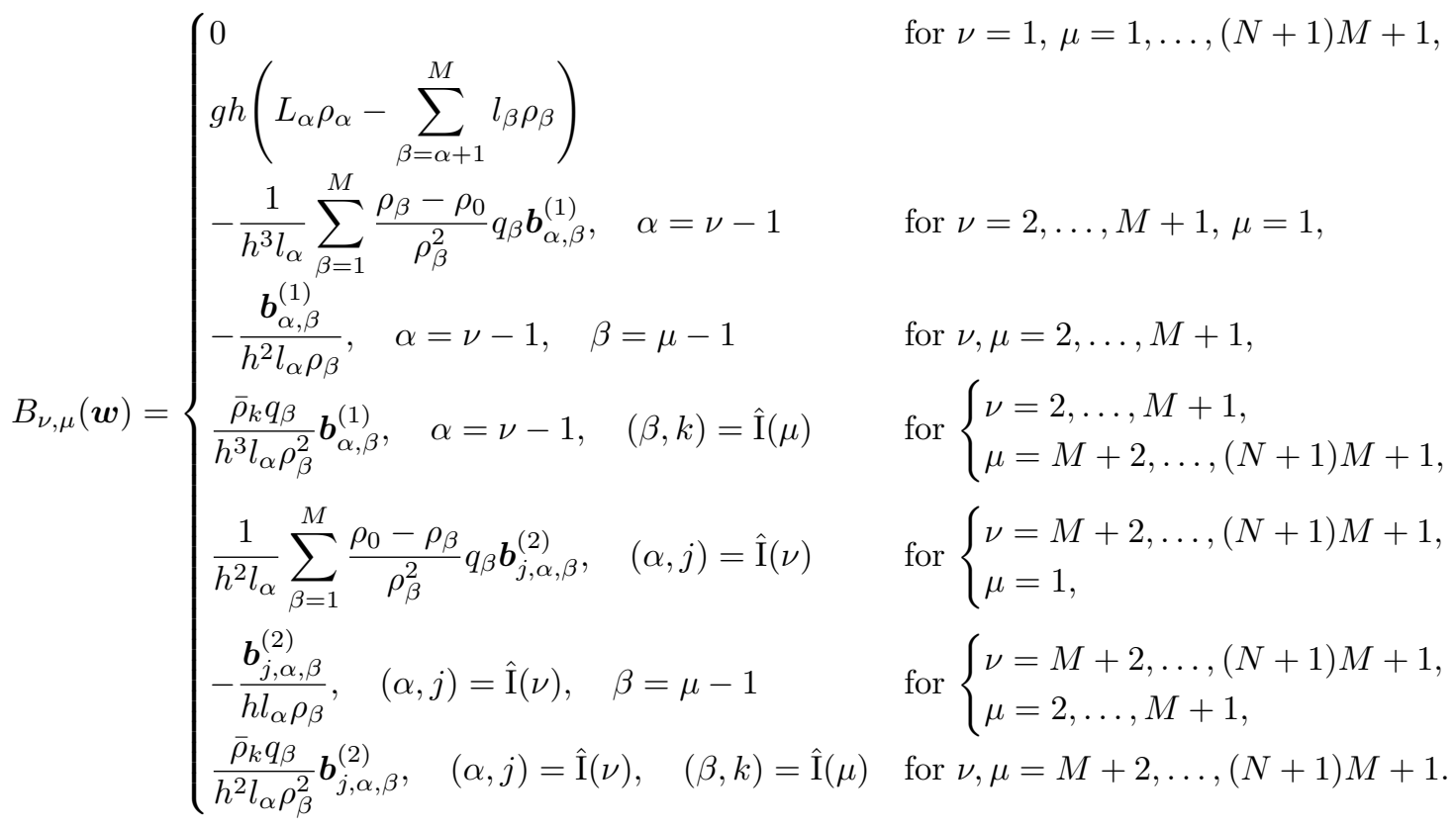

Equation (3.14) also can be rewritten in the form

$$
\partial_{t} \boldsymbol{w}+\boldsymbol{A}(\boldsymbol{w}) \partial_{x} \boldsymbol{w}=\boldsymbol{S}(\boldsymbol{w}) \partial_{x} H+\boldsymbol{G}(\boldsymbol{w}),
$$

where $\boldsymbol{A}(\boldsymbol{w})=\boldsymbol{B}(\boldsymbol{w})+\boldsymbol{J}(\boldsymbol{w})$, and $\boldsymbol{J}(\boldsymbol{w})=\partial \boldsymbol{F}(\boldsymbol{w}) / \partial \boldsymbol{w}$ is the Jacobian matrix of $\boldsymbol{F}$. We define, 
below, the Jacobian matrix $\boldsymbol{J}(\boldsymbol{w})=\left(J_{\nu, \mu}(\boldsymbol{w})\right)_{\nu, \mu=1, \ldots,(N+1) M+1} \in \mathcal{M}_{(N+1) M+1}(\mathbb{R})$.

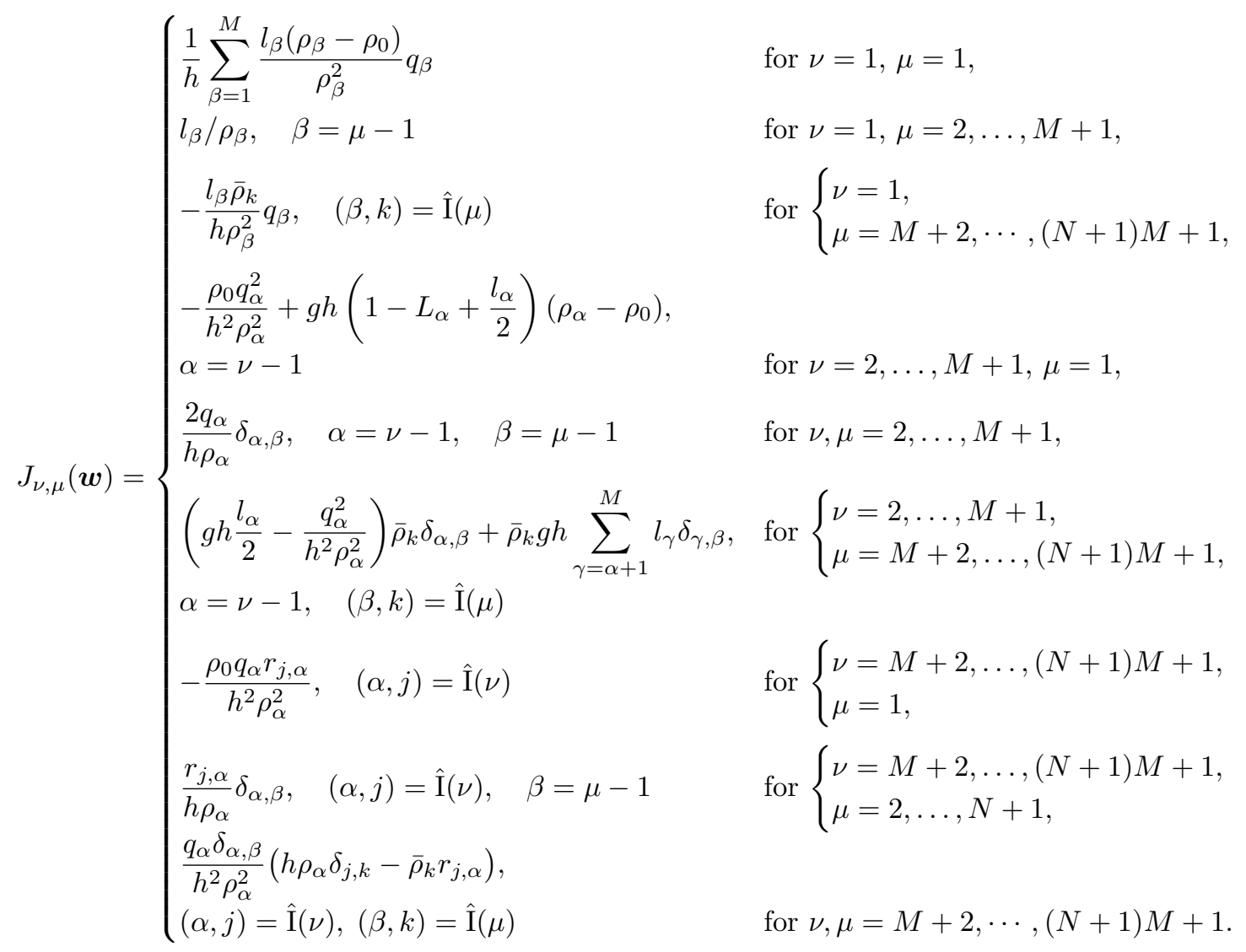

\section{B Appendix: definition in terms of the variable $\widetilde{w}$}

With the unknown vector $\widetilde{\boldsymbol{w}}$ in (4.4), the first equation of system (4.2) is merely rewritten as

$$
\partial_{t} h+\partial_{x}\left(\sum_{\beta=1}^{M} l_{\beta} \frac{h q_{\beta}}{m_{\beta}}\right)=0 .
$$

Next, considering the expression (3.11), we have

$$
\partial_{x}\left(h u_{\gamma}\right)=\frac{1}{\rho_{\gamma}(\Phi)} \partial_{x} q_{\gamma}-\frac{q_{\gamma}}{h \rho_{\gamma}^{2}(\Phi)} \partial_{x}\left(h \rho_{\gamma}(\Phi)\right)+\frac{q_{\gamma}}{h \rho_{\gamma}(\Phi)} \partial_{x} h=\frac{h}{m_{\gamma}} \partial_{x} q_{\gamma}-\frac{h q_{\gamma}}{m_{\gamma}^{2}} \partial_{x}\left(m_{\gamma}\right)+\frac{q_{\gamma}}{m_{\gamma}} \partial_{x} h,
$$

and then we exploit the previous computations (Appendix A) for the case of system (3.10) to complete the compact form of (4.2). More precisely, we rewrite the second and third parts of the system respectively as

$$
\begin{aligned}
& \partial_{t} m_{\alpha}+\partial_{x} q_{\alpha}-\sum_{\gamma=1}^{M} \frac{1}{h l_{\alpha}} \Delta_{-}^{\alpha}\left(\xi_{\alpha, \gamma} m_{\alpha+\frac{1}{\gamma} 2}\right)\left(\frac{h}{m_{\gamma}} \partial_{x} q_{\gamma}-\frac{h q_{\gamma}}{m_{\gamma}^{2}} \partial_{x}\left(m_{\gamma}\right)+\frac{q_{\gamma}}{m_{\gamma}} \partial_{x} h\right) \\
& =-\sum_{j=1}^{N} \frac{\bar{\rho}_{j}}{l_{\alpha}} \Delta_{-}^{\alpha} f_{j, \alpha+1 / 2}(\Phi)
\end{aligned}
$$


and

$$
\begin{aligned}
& \partial_{t} q_{\alpha}+\partial_{x}\left(\frac{q_{\alpha}^{2}}{m_{\alpha}}+g h\left[\frac{1}{2} l_{\alpha} m_{\alpha}+\sum_{\beta=\alpha+1}^{M} l_{\beta} m_{\beta}\right]\right)+g\left(L_{\alpha-1} m_{\alpha}-\sum_{\beta=\alpha+1}^{M} l_{\beta} m_{\beta}\right) \partial_{x} h \\
& -\sum_{\gamma=1}^{M} \frac{1}{h l_{\alpha}} \Delta_{-}^{\alpha}\left(\xi_{\alpha, \gamma} q_{\alpha+1 / 2}\right)\left(\frac{h}{m_{\gamma}} \partial_{x} q_{\gamma}-\frac{h q_{\gamma}}{m_{\gamma}^{2}} \partial_{x}\left(m_{\gamma}\right)+\frac{q_{\gamma}}{m_{\gamma}} \partial_{x} h\right) \\
& =-\sum_{j=1}^{N} \frac{\bar{\rho}_{j}}{l_{\alpha}} \Delta_{-}^{\alpha}\left(\frac{q_{\alpha+1 / 2}}{m_{\alpha+1 / 2}} f_{j, \alpha+1 / 2}(\Phi)\right)-g m_{\alpha} \partial_{x} z_{\mathrm{B}}-h \partial_{x} p_{\mathrm{S}}
\end{aligned}
$$

Here also, the system of $2 M+1$ equations made up of the equations (B.1), (B.2) and (B.3) has the form (4.3) with the notation (4.4) for the unknown vector. The scalar function $\widetilde{H}$ still represents the bottom function. The vectors $\widetilde{\boldsymbol{S}}(\widetilde{\boldsymbol{w}})=\left(\widetilde{S}_{\nu}(\widetilde{\boldsymbol{w}})\right)_{\nu=1, \ldots, 2 M+1}, \widetilde{\boldsymbol{G}}(\widetilde{\boldsymbol{w}})=\left(\widetilde{G}_{\nu}(\widetilde{\boldsymbol{w}})\right)_{\nu=1, \ldots, 2 M+1}$ and $\widetilde{\boldsymbol{F}}(\widetilde{\boldsymbol{w}})=\left(\widetilde{F}_{\nu}(\widetilde{\boldsymbol{w}})\right)_{\nu=1, \ldots, 2 M+1}$ are given by the respective expressions

$$
\begin{gathered}
\tilde{S}_{\nu}(\widetilde{\boldsymbol{w}})=\left\{\begin{array}{lr}
0 & \text { for } \nu=1, \ldots, M+1, \\
-g m_{\alpha}, \alpha=\nu-M-1 & \text { for } n=M+2, \cdots, 2 M+1,
\end{array}\right. \\
\widetilde{G}_{\nu}(\widetilde{\boldsymbol{w}})=\left\{\begin{array}{lr}
G_{M+1 / 2}-G_{1 / 2} & \text { for } \nu=1, \\
-\sum_{j=1}^{N} \frac{\bar{\rho}_{j}}{l_{\alpha}} \Delta_{-}^{\alpha} f_{j, \alpha+1 / 2}(\Phi), \alpha=\nu-1 & \text { for } \nu=2, \ldots, M+1, \\
-\sum_{j=1}^{N} \frac{\bar{\rho}_{j}}{l_{\alpha}} \Delta_{-}^{\alpha}\left(\frac{q_{\alpha+1 / 2}}{m_{\alpha+1 / 2}} f_{j, \alpha+1 / 2}(\Phi)\right)-h \partial_{x} p_{\mathrm{S}}, & \text { for } \nu=M+2, \ldots, 2 M+1, \\
\alpha=\nu-M-1 & \text { for } \nu=1,
\end{array}\right. \\
\widetilde{F}_{\nu}(\widetilde{\boldsymbol{w}})=\left\{\begin{array}{rr}
M & \text { for } \nu=2, \ldots, M+1, \\
\sum_{\beta=1}^{M} l_{\beta} \frac{h q_{\beta}}{m_{\beta}} \quad \alpha=\nu-1 & \text { for } \nu=M+2, \ldots, 2 M+1 . \\
\frac{q_{\alpha}^{2}}{m_{\alpha}}+g h\left(\frac{1}{2} l_{\alpha} m_{\alpha}+\sum_{\beta=\alpha+1}^{M} l_{\beta} m_{\beta}\right), & \alpha=\nu-M-1
\end{array}\right.
\end{gathered}
$$


The matrix $\widetilde{\boldsymbol{B}}(\widetilde{\boldsymbol{w}})=\left(\widetilde{B}_{\nu, \mu}(\widetilde{\boldsymbol{w}})\right)_{\nu, \mu=1, \ldots, 2 M+1}$ is defined by

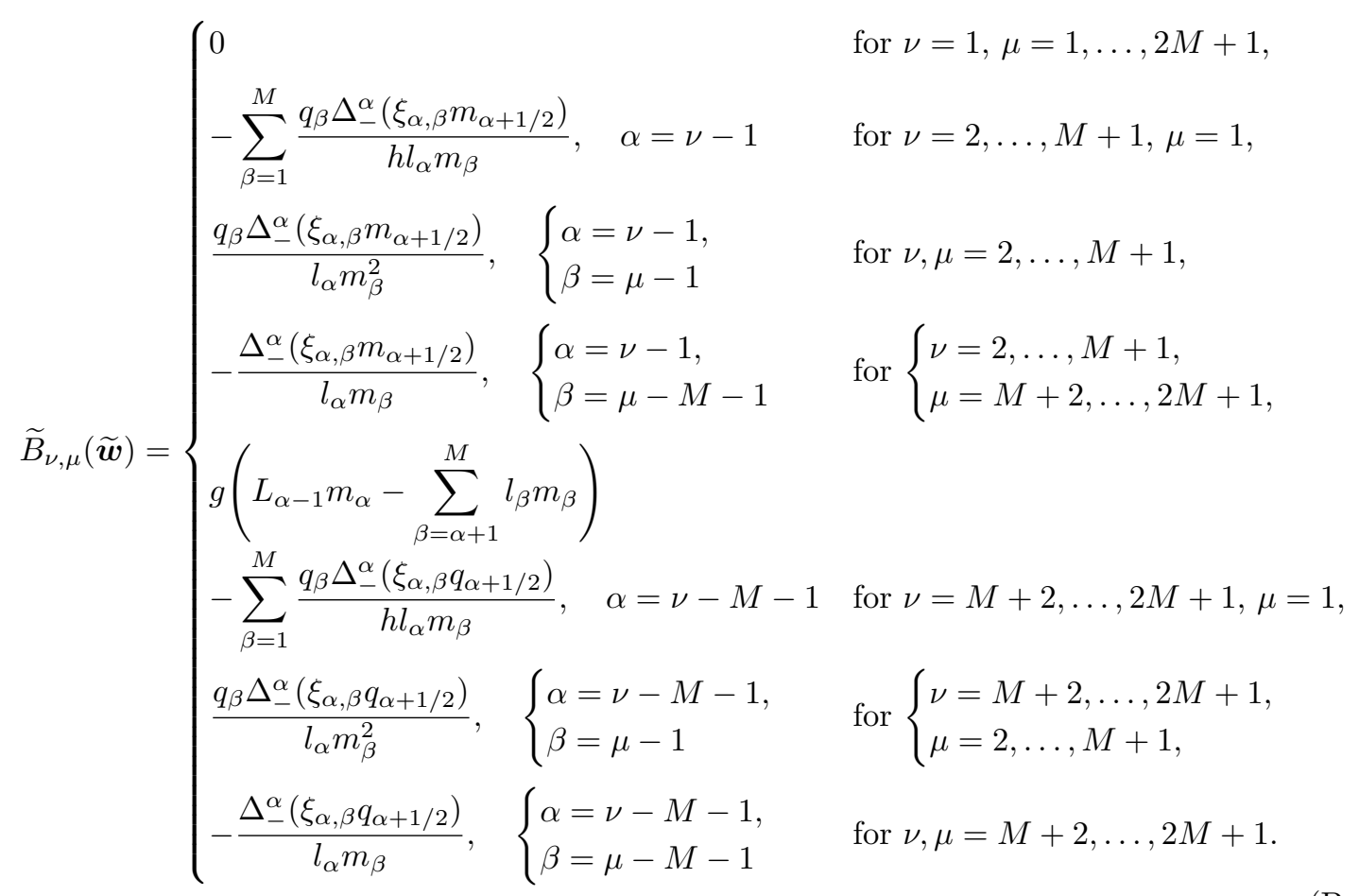

Here again, the form (4.3) can be rewritten as

$$
\partial_{t} \widetilde{\boldsymbol{w}}+\widetilde{\boldsymbol{A}}(\widetilde{\boldsymbol{w}}) \partial_{x} \widetilde{\boldsymbol{w}}=\widetilde{\boldsymbol{S}}(\widetilde{\boldsymbol{w}}) \partial_{x} \widetilde{H}+\widetilde{\boldsymbol{G}}(\widetilde{\boldsymbol{w}}),
$$

where $\widetilde{\boldsymbol{A}}(\widetilde{\boldsymbol{w}})=\widetilde{\boldsymbol{B}}(\widetilde{\boldsymbol{w}})+\widetilde{\boldsymbol{J}}(\widetilde{\boldsymbol{w}})$ and $\widetilde{\boldsymbol{J}}(\widetilde{\boldsymbol{w}})=\partial \widetilde{\boldsymbol{F}}(\widetilde{\boldsymbol{w}}) / \partial \widetilde{\boldsymbol{w}}$ is the Jacobian matrix of $\widetilde{\boldsymbol{F}}$. The matrix 


$$
\begin{aligned}
& \widetilde{\boldsymbol{J}}(\widetilde{\boldsymbol{w}}) \in \mathcal{M}_{2 M+1}(\mathbb{R})=\left(\widetilde{J}_{\nu, \mu}(\widetilde{\boldsymbol{w}})\right)_{\nu, \mu=1, \ldots, 2 M+1} \text { is then given below. } \\
& \widetilde{J}_{\nu, \mu}(\widetilde{\boldsymbol{w}})= \begin{cases}\sum_{\beta=1}^{M} l_{\beta} \frac{q_{\beta}}{m_{\beta}} & \text { for } \nu, \mu=1, \\
-l_{\beta} \frac{h q_{\beta}}{m_{\beta}^{2}}, \beta=\mu-1 & \text { for } \nu=1, \mu=2, \ldots, M+1, \\
l_{\beta} \frac{h}{m_{\beta}}, \beta=\mu-M-1 & \text { for } \nu=1, \mu=M+2, \ldots, 2 M+1, \\
0 & \text { for } \nu=2, \ldots, M+1, \mu=1, \ldots, M+1, \\
q_{\alpha} \delta_{\alpha, \beta}, \alpha=\nu-1, \beta=\mu-M-1 & \text { for }\left\{\begin{array}{l}
\nu=2, \ldots, M+1, \\
\mu=M+2, \ldots, 2 M+1,
\end{array}\right. \\
\frac{1}{2} g l_{\alpha} m_{\alpha}+g \sum_{\beta=\alpha+1}^{M} l_{\beta} m_{\beta}, \alpha=\nu-M-1 & \text { for } \nu=M+2, \ldots, 2 M+1, \mu=1, \\
-\frac{q_{\alpha}^{2}}{m_{\alpha}^{2}} \delta_{\alpha, \beta}+g h\left(\frac{l_{\alpha} \delta_{\alpha, \beta}}{2}+\sum_{\gamma=\alpha+1}^{M} l_{\gamma} \delta_{\gamma, \beta}\right), & \\
\alpha=\nu-M-1, \beta=\mu-1 & \text { for }\left\{\begin{array}{l}
\nu=M+2, \ldots, 2 M+1, \\
\mu=2, \ldots, M+1,
\end{array}\right. \\
\frac{2 q_{\alpha}}{m_{\alpha}} \delta_{\alpha, \beta}, \alpha=\nu-M-1, \beta=\mu-M-1 & \text { for } \nu, \mu=M+2, \ldots, 2 M+1 .\end{cases}
\end{aligned}
$$

\section{Acknowledgements}

RB acknowledges support by Fondecyt project 1090456, BASAL project PFB03 Centro de Modelamiento Matemático (U. de Chile)/CI²MA (U. de Concepción), and CONICYT project Anillo ACT1118 (ANANUM).

TML acknowledges support by the Spanish Government and FEDER through the Research projects MTM2009-11923, and by the Andalusian Government through the project P11- RNM7069.

EDFN and EHK acknowledge support by the Spanish Government and FEDER through the Research project MTM2009-07719.

\section{References}

[1] E. Audusse. A multilayer Saint-Venant model: derivation and numerical validation. Discrete Contin. Dyn. Syst. Ser. B, 5(2):189-214, 2005.

[2] E. Audusse, M. Bristeau, and A. Decoene. 3D free surface flows simulations using a multilayer saint-venant model. comparisons with Navier-Stokes solutions. In A. B. de Castro, D. Gómez, P. Quintela, and P. Salgado, editors, Numerical Mathematics and Advanced Applications, pages 181-189. Springer Berlin Heidelberg, Berlin, Heidelberg.

[3] E. Audusse and M.-O. Bristeau. Finite-volume solvers for a multilayer Saint-Venant system. Int. J. Appl. Math. Comput. Sci., 17(3):311-319, 2007.

[4] E. Audusse, M. O. Bristeau, and A. Decoene. Numerical simulations of 3D free surface flows by a multilayer Saint-Venant model. Internat. J. Numer. Methods Fluids, 56(3):331-350, 2008. 
[5] E. Audusse, M.-O. Bristeau, M. Pelanti, and J. Sainte-Marie. Approximation of the hydrostatic Navier-Stokes system for density stratified flows by a multilayer model: kinetic interpretation and numerical solution. J. Comput. Phys., 230(9):3453-3478, 2011.

[6] E. Audusse, M.-O. Bristeau, B. Perthame, and J. Sainte-Marie. A multilayer Saint-Venant system with mass exchanges for shallow water flows. Derivation and numerical validation. ESAIM Math. Model. Numer. Anal., 45(1):169-200, 2011.

[7] S. Berres and R. Bürger. On Riemann problems and front tracking for a model of sedimentation of polydisperse suspensions. ZAMM Z. Angew. Math. Mech., 87(10):665-691, 2007.

[8] S. Berres, R. Bürger, K. H. Karlsen, and E. M. Tory. Strongly degenerate parabolic-hyperbolic systems modeling polydisperse sedimentation with compression. SIAM Journal on Applied Mathematics, 64(1):41-80, 2003.

[9] R. T. Bonnecaze, H. E. Huppert, and J. R. Lister. Particle-driven gravity currents. J. Fluid Mech., 250:339-369, 1993.

[10] R. Bürger, R. Donat, P. Mulet, and C. A. Vega. Hyperbolicity analysis of polydisperse sedimentation models via a secular equation for the flux Jacobian. SIAM Journal on Applied Mathematics, 70(7):2186-2213, 2010.

[11] R. Bürger, R. Donat, P. Mulet, and C. A. Vega. On the implementation of WENO schemes for a class of polydisperse sedimentation models. J. Comput. Phys., 230(6):2322-2344, 2011.

[12] R. Bürger, A. García, K. H. Karlsen, and J. D. Towers. A family of numerical schemes for kinematic flows with discontinuous flux. J. Engrg. Math., 60(3-4):387-425, 2008.

[13] R. Bürger, K. H. Karlsen, E. M. Tory, and W. L. Wendland. Model equations and instability regions for the sedimentation of polydisperse suspensions of spheres. ZAMM Zeitschrift für Angewandte Mathematik und Mechanik, 82:699-722, 2002.

[14] S. S. S. Cardoso and A. W. Woods. On convection and mixing driven by sedimentation. J. Fluid Mech., 285:165-180, 1995.

[15] M. J. Castro Díaz and E. D. Fernández-Nieto. A class of computationally fast first order finite volume solvers: Pvm methods. SIAM J. Sci. Comput., 34(4):2173-2196, 2012.

[16] M. J. Castro Díaz, E. D. Fernández-Nieto, A. M. Ferreiro, and C. Parés. Two-dimensional sediment transport models in shallow water equations. A second order finite volume approach on unstructured meshes. Comput. Methods Appl. Mech. Engrg., 198(33-36):2520-2538, 2009.

[17] H. Cho and M. S. Klima. Application of a batch hindered-settling model to dense-medium separations. Coal Preparation, 14(3-4):167-184, 1994.

[18] C. M. Choux and T. H. Druitt. Analogue study of particle segregation in pyroclastic density currents, with implications for the emplacement mechanisms of large ignimbrites. Sedimentology, 49(-):907-928, 2002.

[19] G. Dal Maso, P. G. Lefloch, and F. Murat. Definition and weak stability of nonconservative products. J. Math. Pures Appl. (9), 74(6):483-548, 1995.

[20] R. Donat and P. Mulet. A secular equation for the Jacobian matrix of certain multi-species kinematic flow models. Numer. Methods Partial Differential Equations, 26:159-175, 2010.

[21] E. D. Fernández-Nieto. Modelling and numerical simulation of submarine sediment shallow flows: transport and avalanches. Bol. Soc. Esp. Mat. Apl. S $\overrightarrow{\mathrm{e} M A, ~(49): 83-103, ~} 2009$. 
[22] E. D. Fernández-Nieto and G. Narbona-Reina. Extension of WAF type methods to nonhomogeneous shallow water equations with pollutant. J. Sci. Comput., 36(2):193-217, 2008.

[23] D. Gidaspow. Multiphase Flow and Fluidization: Continuum and Kinetic Theory Descriptions. Academic Press, Jan. 1994.

[24] T. C. Harris, A. J. Hogg, and H. E. Huppert. Polydisperse particle-driven gravity currents. Journal of Fluid Mechanics, 472:333-371, 2002.

[25] H. E. Huppert, R. C. Kerr, J. R. Lister, and J. S. Turner. Convection and particle entrainment driven by differential sedimentation. Journal of Fluid Mechanics, 226:349-369, 1991.

[26] M. J. Lockett and K. S. Bassoon. Sedimentation of binary particle mixtures. Powder Technol., 24(1):1-7, 1979.

[27] J. Masliyah. Hindered settling in a multi-species particle system. Chem. Eng. Sci., 34:1166$1168,1979$.

[28] T. Morales de Luna, M. J. Castro Díaz, C. Parés Madroñal, and E. D. Fernández Nieto. On a shallow water model for the simulation of turbidity currents. Communications in Computational Physics, 6(4):848-882, 2009.

[29] C. Parés. Numerical methods for nonconservative hyperbolic systems: a theoretical framework. SIAM J. Numer. Anal., 44(1):300-321 (electronic), 2006.

[30] C. Parés and M. Castro. On the well-balance property of Roe's method for nonconservative hyperbolic systems. Applications to shallow-water systems. M2AN Math. Model. Numer. Anal., 38(5):821-852, 2004.

[31] J. Richardson and W. Zaki. Sedimentation and fluidization: Part I. Trans. Instn. Chem. Engrs. (London), 32:35-53, 1954.

[32] A. Rushton, A. S. Ward, and R. G. Holdich. Solid-Liquid Filtration and Separation Technology. Jan. 2008.

[33] J. Sainte-Marie. Vertically averaged models for the free surface non-hydrostatic Euler system: derivation and kinetic interpretation. Math. Models Methods Appl. Sci., 21(3):459-490, 2011.

[34] W. Sartory. Three-component analysis of blood sedimentation by the method of characteristics. Mathematical Biosciences, 33(1-2):145-165, 1977.

[35] W. Schneider, G. Anestis, and U. Schaflinger. Sediment composition due to settling of particles of different sizes. Int. J. Multiphase Flow, 11:419-423, 1985.

[36] A. Spannenberg, J. Raven, M. Scarboro, and K. Galvin. Continuous differential sedimentation of a binary suspension. Powder Technology, 88(1):45-50, July 1996.

[37] K. Stamatakis and C. Tien. Batch sedimentation calculations - the effect of compressible sediment. Powder Technol., 72:227-240, 1992.

[38] I. Toumi. A weak formulation of Roe's approximate Riemann solver. J. Comput. Phys., 102(2):360-373, 1992. 